\title{
THE EFFECT OF TRAFFIC-LIGHT LABELS AND TIME PRESSURE ON ESTIMATING KILOCALORIES AND CARBON FOOTPRINT OF FOOD
}

\author{
Luca A. Panzone ${ }^{\mathrm{a}}$, Falko F Sniehotta ${ }^{\mathrm{b}}$, Rob Comber ${ }^{\mathrm{c}}$, Fred Lemke $^{\mathrm{d}}$ \\ ${ }^{a}$ School of Natural and Environmental Science, Newcastle University (UK); email: luca.panzone@newcastle.ac.uk \\ ${ }^{\mathrm{b}}$ Institute for Health and Society, Newcastle University (UK); email: falko.sniehotta@ncl.ac.uk \\ ${ }^{\mathrm{c}}$ RISE Research Institute (Sweden); email: rob.comber@ri.se \\ d Vlerick Business School, Ghent University (Belgium). fred.lemke@,vlerick.com
}

\begin{abstract}
Food consumption decisions require consumers to evaluate the characteristics of products. However, the literature has given limited attention to how consumers determine the impact of food on health (e.g., kilocalories) and on the environment (e.g., carbon footprint). In this exercise, 1,511 consumers categorised 43 food products as healthy/unhealthy and good/bad for the environment, and estimated their kilocalories and carbon footprint, which were known to the investigator. The task was performed either with no stimuli (a control group), under time pressure only, with traffic-light labels only, or both. Results show that traffic-light labels: 1) operate through improvements in knowledge, rather than facilitating information processing under pressure; 2) improve the ability to rank products by both kilocalories and carbon footprint, rather than the ability to use the metric; 3 ) reduce the threshold used to categorise products as unhealthy/bad for the environment, whilst raising the threshold used to classify products as good for the environment (but not healthy). Notably, traffic-light increase accuracy by reducing the response compression of the metric scale. The benefits of labels are particularly evident for carbon footprint. Overall, these results indicate that consumers struggle to estimate numerical information, and labels are crucial to ensure consumers make sustainable decisions, particularly for unfamiliar metrics like carbon footprint.
\end{abstract}


Keywords: numerical judgement; sustainable diets; carbon footprint; kilocalories; threshold analysis; multi-level modelling.

\section{Acknowledgements}

The authors acknowledge the support of the Institute for Sustainability, Newcastle University. We are also grateful to Gavin Ellison (YouGov) for invaluable support in the data collection. Discussions with Denis Hilton, Alan Warde, Lucia Rehackova, and Viviana Albani greatly helped improve the final manuscript. 


\section{INTRODUCTION}

Current food consumption in developed economies is often considered unsustainable, harming both peoples' health and the environment (Macdiarmid et al. 2012; Panzone et al. 2018; Springmann et al. 2016). The literature gives significant attention to what motivates sustainable consumption (Panzone et al. 2016b; Prothero et al. 2011; Steg and Vlek 2009), paying less attention to whether consumers can predict the impact of their consumption on health and the environment. However, understanding how consumers determine the consequences of their choices is crucial for policy and practice: selfregulation is only effective if consumers can correctly determine whether they are making the right or wrong decision, as defined by their consumption goals (Baumeister 2002). The inability to correctly self-monitor when making food choices can result in poor health or harm the environment, despite the decision-maker believing otherwise (e.g., Chandon and Wansink 2007a; Chernev 2011a; Chernev 2011b; Faulkner et al. 2014; Gorissen and Weijters 2016; Wansink and Chandon 2006; Whitmarsh, Seyfang, and O’Neill 2011).

\subsection{Food consumption, health, and nutritional traffic-light labels}

Food products are a source of energy and nutrients for humans, and the kilocalories content of a product is often used as an indicator of its healthiness (e.g., Chernev 2011a; Chernev 2011b). In the UK, provision of information on the nutritional content of food was introduced in 1987 to facilitate consumer choices and improve the healthiness of UK diets (Morris 1993). However, while kilocalories have been presented on labels for many years, consumers still struggle when estimating the kilocalories of their meals (e.g. Chandon and Wansink 2007a; Chernev 2011a, b; Shah et al. 2014). The introduction in 2013 of a voluntary scheme for the use of health-focused traffic-light labels by the Department of Health ${ }^{1}$ aimed at further facilitating the identification of healthy food options in the market, presenting complex information in a simple manner by colour-coding in green, amber, or red (e.g. Grunert, Wills, and Fernández-Celemín 2010; Koenigstorfer, Groeppel-Klein, and Kamm

\footnotetext{
${ }^{1}$ See for instance, https://www.food.gov.uk/business-guidance/nutrition-labelling, and https://www.nhs.uk/news/foodand-diet/new-colour-coded-food-nutrition-labels-launched/
} 
2013). The literature shows that traffic-light labels can in fact motivate healthier food choices (Andrews, Burton, and Kees 2011; Koenigstorfer et al. 2013; Osman and Thornton 2019), and make nutrition estimates more accurate (Andrews et al. 2011).

\subsection{Food consumption, environmental sustainability, and environmental traffic-light labels}

Whilst being a key source of nutrients for healthy living, food consumption has very important environmental implications: the production, supply, distribution, and consumption of food emits greenhouse gases (GHG) (Macdiarmid et al. 2012; Panzone et al. 2018; Poore and Nemecek 2018; Springmann et al. 2016), which are often referred to as a product's carbon footprint. Behaviourally, the carbon footprint differs from kilocalories in the impact they have on society: the person who overconsumes kilocalories will directly pay the health consequences of the behaviour (in terms of ill health from e.g., heart disease); while an excessive amount of GHG emitted is reflected in the depletion of the environmental public good locally and globally, affecting negatively also those who consume sustainably. Notably, the carbon footprint of a food product correlates positively, but weakly, with its kilocalories content (Drewnowski et al. 2015). While the UK public is aware of the concept of "carbon footprint" (Whitmarsh et al. 2011), and 2009 data shows that $80 \%$ of UK consumers believe it should be mandatory on labels (European Commission 2009), food products do not report this figure (or an equivalent metric) on labels. As a result, shoppers are often unable to discriminate between high- and low-carbon foods (Gorissen and Weijters 2016; Panzone, Lemke, and Petersen 2016a; Shi et al. 2018; Upham, Dendler, and Bleda 2011; Vanclay et al. 2011). Recent research finds that traffic-light labels based on carbon footprint successfully reduce the environmental impact of food choices (Muller, Lacroix, and Ruffieux 2019; Osman and Thornton 2019).

\subsection{The current article}

This article explores how consumers estimate kilocalories and carbon content of foods normally available in supermarkets, focusing on two aspects of the evaluative process: we observe how consumers categorise products as healthy (vs unhealthy) or good (vs bad) for the environment, while in the second step they were asked to estimate the kilocalories and carbon footprint of the same food 
products. We advance previous research on this topic (Camilleri et al. 2019) by exploring the role of external stimuli on assessments, with some participants doing the task under time pressure, in the presence of a traffic-light label, or both. Specifically, the literature often implicitly understands the effectiveness of traffic-light labels in terms of the decrease in cognitive costs required to process information, a key factor in modern retail environments (some early estimates indicated that choices take an average time of 5 seconds; Blaylock et al. 1999; Moorman 1996). However, the effect of traffic-light labelling might go beyond a reduction in information processing time, and may allow consumers to learn how to use a metric. For instance, health-based traffic-light labels are more effective in consumers who have high nutrition consciousness (Andrews et al. 2011), therefore with an interest on the topic, but with low self-control (Koenigstorfer et al. 2013), therefore requiring the aid of the label. This article advances the literature by examining how traffic light labels operate under time constraint, focusing on kilocalories and carbon footprint estimate (see also Grunert et al. 2010).

\section{HOW CONSUMER MAKE NUMERICAL ESTIMATES}

Estimating an unknown value with precision requires individuals to process a significant amount of information on the characteristics of the item being assessed to obtain a reasonable answer (Simmons, LeBoeuf, and Nelson 2010), often in a limited time. The literature indicates that individuals have a range of plausible values in their mind, and make numerical judgements using a two-stage process (Simmons et al. 2010; Tversky and Kahneman 1974). In the first stage (anchoring), the individual decides whether the correct value is above or below a threshold (self- or experimenter-generated), known as anchor (Epley and Gilovich 2006; Mochon and Frederick 2013; Simmons et al. 2010). For instance, a consumer may believe that $100 \mathrm{~g}$ of spaghetti contain less than the $600 \mathrm{kcal}$ stated by an anchor. This anchor can be semantic: the consumer may believe the $100 \mathrm{~g}$ of spaghetti is "healthy" (the anchor), and the plausible range of calories values only contains low values (Chernev 2011b). In the second stage (adjustment), the individual processes information from memory or provided during the task to identify the exact value (Tversky and Kahneman 1974). For instance, the consumer may expect that $100 \mathrm{~g}$ of spaghetti is certainly between $200-500 \mathrm{kcal}$ and will aim at finding the correct 
answer (360 kcal) in that range. The second stage also uses knowledge activated in the first stage, a phenomenon known as selective accessibility (Mussweiler and Strack 1999): information retrieved from memory to determine whether, for instance, pasta is healthy also primes the adjustment stage.

The final value can be exact (a "full" adjustment) or imprecise (an "incomplete" adjustment). Research indicates that individuals fail to adjust completely when estimating the carbon footprint of food (Camilleri et al. 2019; Gorissen and Weijters 2016), kilocalories (e.g., Chernev 2011a; Chernev 2011b; Liu et al. 2015), water and energy use (Attari 2014; Attari et al. 2010), and expenditures (Scheibehenne 2019; Ülkümen, Thomas, and Morwitz 2008). The failure to identify the correct value may reflect the lack of motivation to exert cognitive effort when searching for it in the second stage; or the use of an excessively wide range of plausible values, for instance due to lack of knowledge of the metric (Epley and Gilovich 2006; Simmons et al. 2010). Moreover, the over-reliance on anchorspecific information (e.g., what makes a food product healthy) pushes the final estimate towards the anchor (Mussweiler and Strack 1999). See Epley and Gilovich (2006) and Mochon and Frederick (2013) for detailed reviews on this topic. Information available to the consumer can bias both stages: for instance, consumers perceive the same product as healthier if it carries an 'organic' label (Lee et al. 2013; Schuldt and Schwarz 2010); while food sold in a store with a healthy reputation or presented with a healthy side dish is perceived as being a low in kilocalories (Chandon and Wansink 2007a; Chernev 2011a) and GHG (Gorissen and Weijters 2016).

As inaccurate estimates can originate from imperfect reasoning in either of the two stages, the analysis of both steps allows for a richer understanding of the process consumers use to determine the unobservable quality of products. In fact, the analysis of a categorisation task where individual classify foods as good or bad for health or the environment, allows estimating the thresholds consumers use to classify products. The analysis of the final assessments allows measuring accuracy by comparing the actual and the estimated value. These points are detailed in the next subsections. 


\subsection{Classifications and metric thresholds}

The categorisation task can be seen as the representation of an underlying mental process whereby a participants place a product into a category (e.g., good or bad for the environment) whenever they expects the underlying metric to be above or below a certain threshold (Bi and Ennis 1998; Lawless and Heymann 2010). This threshold identifies the implicit range of values that is considered plausible (Epley and Gilovich 2006). For instance, a consumer may think that any product is "good for the environment" as long as it emits less than say $600 \mathrm{gCO}_{2}$. We call this an implicit threshold - implicit because it is derived indirectly from observing both stages of the evaluative process, and may differ from the threshold a respondents would report explicitly if requested. The task identifies one threshold below which products are considered "positive" (healthy/good for the environment) with certainty; and one threshold above which products are considered "negative" (unhealthy/bad for the environment) with certainty. Between these thresholds there may be an interval of uncertainty, where participants assign a large probability of the product being in either categories (Wang 1997). The use of actual data then reveals the actual threshold used. For example, a consumer might classify a product as "good for the environment", expecting it to be below $500 \mathrm{gCO}_{2}$; however, if its real carbon footprint is $700 \mathrm{gCO}_{2}$, the real threshold being used to classify products as " $\mathrm{good}$ " is above $700 \mathrm{gCO}_{2}$.

\subsection{Numerical assessments, and metric accuracy}

Conditional on the categorisation task, the final numerical assessment provides the final estimate of the metric under consideration. A comparison between this final value and the actual value determines a measure of accuracy of the assessments. Inaccuracy is caused by two main problems (Hertwig, Pachur, and Kurzenhäuser 2005; Smith and Windschitl 2015): lack of the metric knowledge, or lack of mapping knowledge of respondents. Metric knowledge refers to the ability to assign reasonable values to a metric; in this exercise, it indicates whether individuals can determine the carbon footprint or kilocalories of a food item they see. Mapping knowledge is a measure of the ability of the consumer to compare and rank items; for instance, a consumer with good mapping knowledge would be able to determine that, in general, pasta has more calories per $100 \mathrm{~g}$ than carrots, but less than ready meals. 
At the same time, the comparison between an estimate and the actual value of a metric allows observing whether respondents systematically over- or underestimate the metric: research indicates that individuals underestimate energy (Attari et al. 2010) and water use (Attari 2014), and the carbon footprint of foods (Camilleri et al. 2019); and underestimate (overestimate) the kilocalories content of food sold in restaurants with a reputation for selling healthy (unhealthy) food (Chernev 2011a, b).

\subsection{Testable hypotheses}

\subsubsection{Effect of traffic-light labelling on assessments}

Labels are a key tool to support consumers in the task of estimating the impact of a food product. In particular, the use of colour-coding in traffic-light labelling facilitates the processing of complex information, leading to more sustainable and healthier choices (Andrews et al. 2011; Koenigstorfer et al. 2013; Osman and Thornton 2019; VanEpps, Downs, and Loewenstein 2015). The effectiveness of labels in driving low-carbon choices is partly due to their ability to help consumers estimate the carbon footprint of food product (Camilleri et al. 2019). As a result, labels might help consumers by removing psycho-physical biases that affect perceptions, for instance reducing the extent of a systematic over- or underestimation of a metric (Attari et al. 2010; Chandon and Wansink 2007b); see section 3.6.3 for a detail description of these biases. Hypothesis 1a then is:

Hypothesis 1a: Traffic-light labels improve the accuracy of estimates, reducing the occurrence of psycho-physical estimation biases.

This information may also shift the implicit thresholds used in the categorisation task: external information (health claim) has been shown to shift sensory thresholds for sugar (Chiou, Yeh, and Chang 2009), and the same effects might be observed for categorisation thresholds. Hypothesis $1 \mathrm{~b}$ is:

Hypothesis 1b: Traffic light labels move the implicit thresholds closer to the actual thresholds, reducing the area of uncertainty. 


\subsubsection{Effect of time pressure on assessments}

Information processing time is an important constraint on the ability to make accurate numerical judgements. The effect of time pressure depends on its intensity: moderate time pressure improves accuracy by increasing information processing efficiency (i.e., reducing attention to information with limited relevance to the task); while high time pressure imposes a cognitive load that impairs the ability to process task-relevant information (Suri and Monroe 2003). Under significant time pressure, as in this article, consumers struggle to compare options in a choice set (Reutskaja et al. 2011), and spend less time looking at complex task-relevant information (van Herpen and Trijp 2011). As a result, under time pressure and cognitive load, individuals adjust less (Epley and Gilovich 2006; Suri and Monroe 2003), which in psycho-physical terms implies stronger estimation biases (Attari 2014; Attari et al. 2010; Chandon and Wansink 2007b; Hertwig et al. 2005). Hypothesis 2a then is:

Hypothesis 2a: Time pressure reduces the accuracy of estimates, increasing the strength of psycho-physical estimation biases.

To our knowledge there are no studies exploring the impact of time pressure on perceptual thresholds (see also the discussion in Chiou et al. 2009). The literature implicitly assumes that the range of plausible values is stable (Epley and Gilovich 2006; Simmons et al. 2010), and only changes when new information is available (see section 2.3.1) or new knowledge is generated (Mussweiler and Strack 2000a, b). The self-generated anchor may change under time pressure, but through a different process: for instance, a respondent may classify pasta as unhealthy under time pressure because the time constraint shifts the attention to (away from) information consistent with a high (low) range of plausible value, without changing the range itself. We therefore posit the following hypotheses:

Hypothesis 2b: Time pressure does not change the average thresholds used, nor the distance between implicit and the actual thresholds. 


\subsubsection{Joint effect of traffic-light labelling and time pressure on assessments}

Part of the beneficial effect of the introduction of a traffic-light label is to reduce the time needed to process complex information by providing simple stimuli (Andrews et al. 2011; Koenigstorfer et al. 2013; VanEpps et al. 2015). However, the extent to which the partial relaxation of time pressure is associated to the improvements brought by traffic-light labels is unclear. If traffic-light labels only operate by making time-efficient choices, then the distortive effect of time pressure should remove all the beneficial effect of traffic-light labels. Conversely, if time pressure does not remove all the benefits of traffic-light labels, then labels operate through some other pathways, e.g. learning, which may be the only pathway if the addition of time pressure does not have an impact on the effectiveness of traffic-light labels. Previous research indicates that consumers respond to time pressure by paying more attention to images on labels, and less to textual information (Pieters and Warlop 1999); therefore, the benefits of traffic-light labels may not fully erode under time pressure as consumer attention shifts to easily accessible task-relevant information. The final hypothesis then is:

Hypothesis 3: Time pressure reduces the benefit provided by traffic-light labels, leading to estimates that are less accurate compared to the presence of these labels alone, and implicit thresholds that are further away from the actual thresholds.

\section{METHOD}

\subsection{Overview of the process}

The exercise presented in this article started with a short and simple introduction of kilocalories and carbon footprint, to ensure all participants had a basic understanding of both metrics. This step was important because in the UK food labels report kilocalories, but not carbon footprint. Participants then had to categorise products as healthy/unhealthy, and good/bad for the environment; and to estimate their carbon footprint and kilocalories (the order of these two metrics was randomised). Finally, participants reported their attitudes and beliefs on health and environmental preservation. The full questionnaire can be found in the online appendix 1. The details of each task are explained in the remainder of this section. 


\subsection{Assessment task}

Participants were presented with a list of 43 food products from 13 'aisles' (baked beans, butter, carrots, cola, cucumber, eggs, frozen peas, milk, mushrooms, olive oil, pasta, ready meals, soya milk), chosen from a list of products sold in UK stores ${ }^{2}$ for which the true kilocalories and carbon footprint (cradle-to-grave) were known; the Spearman correlation between actual calories and carbon footprint in this sample of foods is $0.54(\mathrm{p}<0.001)$. We included categories that included a baseline version of the product (i.e., without any claim), plus at least one specific label (e.g., organic) alongside it (table 1). As an example, the spaghetti aisle presented a baseline product (spaghetti $500 \mathrm{~g}$ ), which consumers could compare against a healthy version (whole wheat spaghetti 500g), a low-cost version (value spaghetti, 500g), and an version with a quality claim (organic spaghetti $500 \mathrm{~g}$ ). Fresh meat, a current important area of inquiry (Hartmann and Siegrist 2017; Poore and Nemecek 2018) was not included as the data from the same source was not available at the time of this exercise; however, the list of products included meat-based ready meals and animal products. Participants were given the true name of the product appearing on the online website (see Appendix 1), removing the own brand name, and were told that "all products are currently sold in a large UK supermarket chain using their own brand".

For each product, respondents had to indicate the perceived level of sustainability and health using a 3-point scale: 'Healthy'/'Good for the environment'; 'Unhealthy'/'Bad for the environment'; or 'Unsure'. At the same time, participants made an assessment of the footprint and calorific content of foods per $100 \mathrm{~g}$ of these products (to make comparisons easier, as products differed in size). Participants could count on two aids: firstly, on the top of the page there was a reference table showing information of the metrics for a range of foods not in the assessment list; secondly, participants were told the maximum for each metric $\left(1000 \mathrm{kcal} / 100 \mathrm{~g} ; 1040 \mathrm{gCO}_{2} \mathrm{e} / 100 \mathrm{~g}\right)$. Consumers made these two evaluations one category at a time, with all the products within a category listed in a table. Participants

\footnotetext{
${ }^{2}$ See https://issuu.com/thema1/docs/tesco_product_carbon_footprint_summary_1_. Note that while the data is collected by Tesco Plc, they have not participated to this study.
} 
started by estimating either kilocalories for all categories sequentially (in a random order); or the carbon footprint for all categories sequentially (in a random order). The order was randomised across individuals to avoid consumers systematically avoiding the evaluation of the products presented towards the end of the exercise.

\subsection{Experimental design}

The study is based on a 2 (labelling vs no labelling) x 2 (time pressure vs no time pressure) betweensubjects experimental design. Participants made evaluations after being randomly allocated to one of four experimental conditions below.

\subsubsection{Control group}

In the control condition, consumers assessed products without any additional information or stimuli.

\subsubsection{Response stimuli - Time pressure}

In the 'time pressure' (TP) condition, respondents were presented with a prominently visible timer on a yellow background on the top of each page (each page contained no more than 6 products) with the statement "It is important that you answer as fast as you can. It should not take more than $X$ seconds to answer the questions on this page" (similar to Lamberts 1995), with $X$ calculated as 5 seconds per product on the page. The 5 -second per product limit follows the early mean estimates in the literature (Blaylock et al. 1999; Moorman 1996). Questionnaire testing and a pilot showed that completing each evaluation under 5 seconds was enough to impose significant time pressure ${ }^{3}$, and the 5 -seconds limit was not enforced to prevent dropouts ${ }^{4}$. The manipulation was effective in motivating faster assessments compared to the control group, as shown in section 3.4.

\footnotetext{
${ }^{3}$ In the questionnaire design phase, 10 participants reported being able to complete the 43 assessments within a $3 \mathrm{~m} 35 \mathrm{~s}$ limit ( $5 \mathrm{~s}$ per assessment). A subsequent pilot with 53 respondents from the study population showed that the 30 participants under pressure took a median time of $8.28 \mathrm{~s}$ per assessment (average: $8.97 \mathrm{~s}$ ).

${ }^{4}$ In the time pressure treatment, $80.8 \%$ of kilocalories assessments, and $76.6 \%$ for carbon footprint assessments took more than 5 seconds; in the control group these values were $87.6 \%$ for kilocalories and $83.4 \%$ for carbon footprint.
} 


\subsubsection{Response stimuli - Labelling}

In the 'labelling' (L) condition, respondents made assessments whilst having access to traffic-light labels. Examples of labels can be found in Figure 1, and the full set of labels can be found in online Appendix 2. For kilocalories, the traffic-light label replicated the actual nutritional label of these products in store, omitting kilocalories. For carbon footprint, the label reported the contribution of different stages of the production process (raw materials, manufacture, transport, and retail) ${ }^{5}$ to the final carbon footprint; here, the colour-coding was determined by identifying whether the contribution was high or low compared to other food products (vegetables have many green lights, as opposed to ready meals, which have many red lights). For instance, in figure 1a the largest contributors of carbon emissions come from processing (extracting cream from milk) and retailing (refrigeration), which are high compared to other foods, while the raw material contribution to production of butter was low (emissions from cow breeding would be shared between meat, and dairy products). Notably, the traffic light labels for carbon footprint and kilocalories in this exercise are slightly different: the nutritional label present colour and numbers (grams of nutrient), which can facilitate the estimation because kilocalories are proportional to the amount of fats, carbohydrates, and proteins; while for carbon footprint the label contains colour and text, because numerical contributions are private information, and not available publicly.

\subsubsection{Response stimuli - Labelling + Time pressure}

A final group presented both traffic-light labelling and time pressure jointly, with the manipulations as described above.

\subsection{Psychometric and demographic information}

After the task, a questionnaire collected personal information. Firstly, we collected attitudes to gauge how positive or negative the participants felt about behaviours that protect the environment, and behaviours that protect personal health (from Cornelissen et al. 2008). Attitudes are generally seen as

\footnotetext{
${ }^{5}$ Own design based on Tesco (2012). Information for the carbon emission label has been determined with the support Dr John Kazer of the Carbon Trust.
} 
an important predictor of behaviour in the literature (Panzone et al. 2016b). Secondly, identity is an important driver of behaviour, because it shows how strongly a goal (e.g., personal health protection, environmental preservation) is central to the sense of self of the individual (Brick, Sherman, and Kim 2017); as a result, health and environmental identity were collected by requesting the level of agreement to the statements "Being (environmentally-friendly/healthy) is an important part of who I am" (from Cornelissen et al. 2008). Attitudes and identity were collected using 5-point scales.

Prior knowledge of a metric may influence the accuracy of estimates (Mussweiler and Strack 2000a); therefore, knowledge of 'healthy' (two questions taken from Parmenter and Wardle 1999) or 'environmentally-friendly' (two questions based on data in Berners-Lee 2011) was collected by asking respondents to identify the product with the highest kilocalories/carbon footprint from a pair (e.g., butter and margarine). An additional control for prior knowledge is the frequency of purchasing food that is healthy or good for the environment; frequency was measured on a 5-point scale by asking how often the participant bought food with a health or environmentally-friendly label in the past 4 weeks (Never; 1-3 times/ month; 1-2 times/month; 3-6 times/week; More often than 6 times per week). The full questionnaire can be found in the online Appendix 1.

\subsection{The sample of participants}

This study collected primary data, where 1,511 individuals representative of the UK population (by gender, age, and region) participated in an online task evaluating 43 food products. Participants were recruited by the polling company YouGov, who managed any compensation and contact with the respondents. No information on the study was provided to participants at any time besides what is reported in Appendix 1. The random allocation of participants targeted around 375 per group; sample sizes by group after removing invalid responses, as explained in detail in section 4.2 , is reported at the bottom of table 2 . 


\subsection{Comparing estimated and actual values}

In this section, we discuss the approach taken to estimate the accuracy of the assessments. We use a suffix $i$ to identify consumers, and a suffix $j$ to characterise products. The task of participants was to give an estimate of a metric, $y$, for a product with actual value, $y^{*}$, which they did not know. The comparison of $y$ and $y^{*}$ provides the basis of the measurement of accuracy.

\subsubsection{Precision}

The precision of participants' estimates are measured as the Root-Mean-Squared-Deviation (RMSD). RMSD measures the average deviation of each assessment from the true value for each individual, and can be interpreted as the average bias of an individual. The RMSD is calculated as $R M S D_{i}=$ $\sqrt{\frac{\sum_{j=1}^{J}\left(y_{i j}-y_{i j}^{*}\right)^{2}}{J}}$. Unbiased estimates correspond to $R M S D=0$.

\subsubsection{Knowledge}

The estimation of the metric and mapping knowledge of respondents follows Hertwig et al. (2005) and Smith and Windschitl (2015).

\subsubsection{Metric knowledge}

Metric knowledge is measured by the Order of Magnitude Error (OME) as $O M E_{i j}=\left|\log _{10}\left(\frac{y_{i j}}{y_{i j}^{*}}\right)\right|$. For instance, it tests whether consumers assigned the correct value of kilocalories to a ready meal. If the estimated value is the same as the actual value, $O M E=0$. The more the OME differs from zero, the poorer is the metric knowledge of an individual. Note that because some values of $y_{j}^{*}$ are zero, we actually calculate the OME as $\left|\log _{10}\left(\frac{y_{i j}+1}{y_{i j}^{*}+1}\right)\right|$ to ensure the ratio is feasible (zero never appears at the denominator), and equals 1 in the case of an exact assessment.

\subsubsection{Mapping knowledge}

Mapping knowledge is measured by a Spearman correlation coefficient $\rho$ between the estimate $y$ and the actual value $y^{*}$. The non-parametric correlation coefficient is preferred to avoid relying on 
normality assumptions; results using Pearson correlations are similar both in the significance of effects and in magnitude. Perfect mapping knowledge corresponds to $\rho=1$.

\subsubsection{Systematic underestimation versus overestimation}

The relationship between estimates and actual values can be further assessed using a multivariate regression that regresses the estimate $y$ of the true value $y^{*}$ as (Attari 2014; Attari et al. 2010)

$$
\log _{10}\left(y_{i j}\right)=\gamma_{0 i}+\gamma_{1 i} \log _{10}\left(y_{j}^{*}\right)+\gamma_{2 i}\left[\log _{10}\left(y_{i j}^{*}\right)\right]^{2}+\varepsilon_{i j}
$$

In equation (1), the three variables are centred by the mean of $\log _{10}\left(y_{i j}^{*}\right)$, to obtain coefficients that directly quantify deviations from the mean of the actual value. This regression compares perceptions and actual values to understand the origin of estimation biases from the coefficients $\gamma_{0 i}, \gamma_{1 i}$, and $\gamma_{2 i}$. Unbiased estimates entail that $\gamma_{0 i}=0, \gamma_{1 i}=1$, and $\gamma_{2 i}=0$. An intercept $\gamma_{0 i} \neq 0$ indicates a baseline bias, a systematic over- or underestimation (on average) of the products. The parameter $\gamma_{1 i} \neq 1$ indicates a proportional bias, whereby consumers expectations assign more $\left(\gamma_{1 i}>1\right)$ or less $\left(\gamma_{1 i}<1\right)$ than one unit increase for each unit increase in the actual value of carbon footprint or kilocalories; in signal detection theory (see Scheibehenne 2019), $\gamma_{1 i}<1$ indicates response compression (the perceived increase in kilocalories or carbon footprint is slower than reality), while $\gamma_{1 i>1}$ indicates response expansion (the perceived increase in the same metrics is faster than reality). Finally, $\gamma_{2 i} \neq 0$ indicates an extreme-value bias, whereby respondents give excessive weight to extreme values at both ends of the scale. In estimating equation (1), we allow for subjective biases, with parameters that are allowed to vary with the experimental group and personal characteristics (plus a random error). More details on this statistical model are reported in Appendix 3.

\subsection{The estimation of categorisation thresholds}

The presence of both a categorisation task and a final numerical estimation task allows estimating the thresholds participants used to determine whether the product is environmentally-friendly and healthy. To do so, we use a binary probit approach that defines a threshold as the point on a metric 
scale where $50 \%$ of participants switch from a category to the other (Bi and Ennis 1998; Lawless and Heymann 2010; Logvinenko, Tyurin, and Sawey 2012). Specifically, we assign a dummy $G_{i j}=1$ if consumer $i$ classifies product $j$ as "Good for the environment" or "Healthy", 0 otherwise; and a dummy $B_{i j}=1$ if a product is classified as "Bad for the environment" or "Unhealthy", 0 otherwise. To estimate the implicit thresholds, we regress these binary assessments against the carbon footprint and the kilocalories participants estimated using two probit equations:

$$
\begin{aligned}
& G_{i j}=\bar{\beta}_{0}^{L}+\delta_{0}^{L} I_{i}+\bar{\beta}_{1}^{L} y_{i j}+\delta_{1}^{L} I_{i} y_{i j}+e_{i j}^{L} \\
& B_{i j}=\bar{\beta}_{0}^{H}+\delta_{0}^{H} I_{i}+\bar{\beta}_{1}^{H} y_{i j}+\delta_{1}^{H} I_{i} y_{i j}+e_{i j}^{H}
\end{aligned}
$$

where $I$ is the experimental group. Following Knoblauch and Maloney (2012, page 154), threshold are estimated as $\bar{y}^{L}=-\left(\frac{\bar{\beta}_{0}^{L}+\delta_{0}}{\bar{\beta}_{1}^{L}+\delta_{1}}\right)$ and $\bar{y}^{H}=-\left(\frac{\bar{\beta}_{0}^{H}+\delta_{0}}{\bar{\beta}_{1}^{H}+\delta_{1}}\right)$ (note $\delta_{0}$ and $\delta_{1}$ are zero at baseline). In the estimation of actual thresholds, $y_{i j}$ in equations (2a) and (2b) is replaced by $y_{i j}^{*}$. More detail on the statistical model is presented in Appendix 3.

\section{RESULTS}

The results of the analyses in sections 3.6 and 3.7 are presented in this section. After starting with the general descriptive statistics of the sample, section 4.2 will discuss the ex-post identification of invalid responses. Section 4.3 will present graphically the relationship between estimates and actual values prior to any quantitative analysis. Section 4.4 then estimates the accuracy of the carbon footprint and kilocalories estimates, testing specifically hypotheses 1a, 2a, and 3a. Section 4.5 then estimates the implicit thresholds consumers used to determine whether a product was positive or negative (in terms of either carbon footprint and kilocalories), testing hypotheses $1 b, 2 b$, and $3 b$. Note that while the two-stage process presented in section 2 starts from a categorisation task, and proceed with a final numerical assessment, here the results are presented in reverse: the initial analysis provides an understanding of the accuracy that characterises the assessments in the sample, which is then complemented by the threshold analysis in the next section. 


\subsection{Descriptive statistics}

The initial sample consists of 1,511 participants (47.3\% men), with an average age of 48.4 years (s.d. $=17.3$, range $=18-95)$. On a 5-point Likert scale, these participants reported relatively strong attitudes in favour of the environment (4.1) and health (3.8), and pro-environmental (3.7) and healthy identity (3.3). When asked to identify the product with the lowest carbon footprint or kilocalories in a pair, participants responded correctly to around 0.8 of 2 questions. Finally, individuals more frequently purchase products with a healthy label as opposed to an environmental label. Summary statistics by experimental condition (Table 2) show that participants took less time to complete all the tasks (categorisation, assessment, and final survey) under time pressure; similarly, individual product evaluations were faster under time pressure for both metrics, with carbon footprint tasks completed slightly faster than kilocalories tasks, except when in the presence of traffic-light labels alone.

\subsection{Detection of insufficient effort responding}

The survey exercise was complex, and had no mechanism in place to prevent insufficient effort responding (IER). The literature reports two key criteria to identify IER observations ex-post (Bowling et al. 2016; Curran 2016; Huang et al. 2012; Meade and Craig 2012): unusual response patterns and low response time; as a result, IER observations were identified in two steps. The first step removed individuals who used less than 7 values for the 43 products $^{6}$ for either carbon footprint or kilocalories. Figure A1 in Appendix 2 shows that the number of different values increases rapidly after this point in both metrics. Importantly, this step removes all the responses a participant gave for a specific metric; however, the participant is dropped entirely only if insufficient effort is detected in both metrics.

The second step removed fast responses within a category: we removed the fourth percentile at the bottom of the distribution (included), using the treatment-specific cut-off points reported in Table

\footnotetext{
${ }^{6}$ This arbitrary value was derived from the number of categories used in the assessment, 13. Some participants may accept that every product within a category has the same carbon or kilocalories content, and allow only limited variability from all products from animal and vegetable origin. As a result, we used as cut-off point the average the integer above the $50 \%$ of categories (13/2). Results are qualitatively consistent if more or less restrictive cut-off points are chosen.
} 
A2 in Appendix 2. This step only removed evaluations within a category where the average response time was below the cut-off point, and only eliminated participants altogether if their estimates were too fast throughout the exercise. The final analyses includes 1,218 participants with an average of 41.25 carbon footprint estimates; and 1,128 participants with an average of 41.09 kilocalories estimates. Table A3 (Appendix 2) reports the demographic characteristics of the final samples, which are very close to the values in table 2 . The results below do not vary in substance if different cut-off points are chosen; however, the exclusion improves the estimate of the variance (e.g., assessments of a participant who used 1000 for all products have zero variance, and bias the estimate of the overall variance downwards).

\subsection{A graphical comparison of estimated and real values}

To observe the relationship between the estimates of participants (valid responses only) and the real values, Figures $2 \mathrm{a}$ and $2 \mathrm{~b}$ plots the (log) median estimate of each product for each metric compared to the $(\log )$ actual value. In these figures, a diagonal dotted line indicates the exact assessments; all products above the dotted lines have been overestimated, while all products below the dotted line have been underestimated. The mean and the $95 \%$ confidence interval for each product are presented in online Appendix 3. Figure 2a shows that carbon footprint estimates are almost orthogonal to actual values (i.e., a horizontal line), both in the control and under time pressure; estimates rotate towards the optimal line when a label is provided (in line with hypothesis $1 \mathrm{a}$ ). Figure $2 \mathrm{~b}$ shows that kilocalories are closer to the dotted line than carbon footprint, an indication that estimates are more precise; notably, both kilocalories and carbon footprint estimates have a quadratic relationship with actual values, but the parabola for kilocalories is downward facing. Labelling again rotates this curve closer to the optimal assessment line (in line with hypothesis 1a).

\subsection{Traffic-light labels improve the accuracy of estimates, while time pressure reduces it}

\subsubsection{Estimation accuracy and knowledge}

Table 3 shows that participants were unable to correctly estimate the carbon footprint and kilocalories of the products provided, showing low levels of accuracy in terms of RMSD (the average bias), OME 
(metric knowledge), and Spearman correlations (mapping knowledge). To interpret these parameters, it is worth recalling that exact assessments correspond to $\operatorname{RMSD}=0, \mathrm{OME}=0$, and $\rho=1$. To test hypotheses 1a, 2a, and 3a, we firstly use a series of Kruskal-Wallis tests to determine whether these assessments vary across experimental condition. In the control group, consumers have a better metric knowledge of kilocalories $(\mathrm{OME}=0.48)$ than carbon footprint $(\mathrm{OME}=0.43)$; kilocalories estimates are also more precise - the RMSD is lower; and products are easier to rank by kilocalories than carbon footprint ( $\rho=0.47$ vs 0.41 ). In the presence of a traffic-light label, these values move towards (but do not reach) an exact assessment, supporting hypothesis 1a: accuracy and knowledge for carbon footprint and kilocalories are much closer, while improvements in OME are small. As a result, trafficlight labels help consumers ranking products, but have a small impact on mapping knowledge. Time pressure has a negative effect on accuracy, mapping and metric knowledge, supporting hypothesis 2a. ANOVA results (Table 4) indicate that changes in accuracy and knowledge are primarily driven by the main effects, with a non-significant contribution of the interaction term, therefore rejecting hypothesis 3: the benefits of traffic-light labels are not associated to a better performance under time pressure, but to other psychological processes (e.g., learning).

\subsubsection{Perception vs reality in the estimation of carbon footprint and kilocalories}

The previous section showed that estimates are more accurate when a traffic-light label is provided. However, it does not explain how perceptions changed in the different groups, which is addressed by estimating equation (1). Results in Table 5 show that respondents have a baseline bias that is positive for kilocalories and negative for carbon footprint: individuals systematically overestimate kilocalories, and underestimate carbon emissions; this bias does not change across groups, an indication that the overall ability to evaluate products remained the same. The proportional bias shows that respondents 'discount' the value of a unit of both metrics: a $1 \%$ increase in the actual value of carbon footprint is perceived as an increase of only $0.26-0.27 \%$ in the estimate, a value raising to $0.36-0.38 \%$ for kilocalories. This is evidence of insensitivity to increases in the value of both metrics, or response compression (Scheibehenne 2019). Crucially, traffic-light labels operate by reducing this 
proportional bias, pushing it closer to the no-bias condition of 1, supporting hypothesis 1a; while time pressure increases it, supporting hypothesis 2a. Finally, the quadratic term indicates that consumers overestimate the carbon footprint of high-carbon foods, and underestimate slightly the caloric content of high energy foods; traffic-light labels reduce this extreme-value bias for carbon footprint only, providing some support to hypothesis 1a. The Wald tests in table 5 indicates that time pressure significantly reduces the ability of traffic-light labels to correct for the proportional bias of both metrics, and the extreme-value bias for carbon footprint only; both results support hypothesis 3 .

The addition of demographics does not alter the significance of these effects, but explain the variability in the estimation biases (table 5, columns 3 and 5). Male participants underestimate carbon footprint more than female participants, and overestimate kilocalories by a smaller amount than females. Men also perceive a unit increase in both metrics to be smaller than what women perceive. Both proportional and extreme-value biases increase with age for both metrics. Knowledge improves the baseline bias for carbon footprint, whilst worsening it for kilocalories; and also improves the proportional bias for both metrics. Individuals scoring high on health identity have a higher extremevalue bias for kilocalories, while individuals with stronger health attitudes have lower extreme-value bias for kilocalories. Finally, people who purchase healthy items more frequently have a lower proportional bias and a lower extreme-value bias when estimating kilocalories only. Personal characteristics do not explain variations in the extreme-value bias for carbon footprint.

\subsection{Traffic light labels bring implicit thresholds closer to the actual thresholds, while time pressure does not change them}

The results in the previous section showed that the traffic-light labels improved the ability to estimate the carbon footprint and kilocalories of food. This section estimates the categorization thresholds participants used in all experimental groups, testing hypotheses $1 b, 2 b$, and $3 b$. As expected (e.g., Lawless and Heymann 2010), the percentage of participants classifying a product as positive (negative) increases (decreases) with the logarithm of carbon footprint (Figure A2a, Appendix 4) and kilocalories (figure A2b, Appendix 4), an indication that assessments reflect the semantic hierarchy: 
consumers coherently expect the environmental friendliness and healthiness of foods to decrease with carbon emissions and kilocalories. Interestingly, estimates did not reach the upper asymptote of the sigmoid curve, suggesting that respondents did not expect products to be critically unhealthy or bad for the environment. Note that the probability of classifying a product as "healthy/unhealthy" and "good/bad for the environment" varied across food category: the raw probabilities, presented in Table A4 in Appendix 4, shows that in some categories (e.g., vegetables or eggs), products are very rarely classified as unhealthy (less than $2 \%$ in the case of vegetables) or bad for the environment; the opposite applies for other products (e.g., ready meals), which are generally considered negatively.

The thresholds (implicit and actual) used by respondents are presented graphically in Figure 3; their values are reported in Table 6. In the graph for kilocalories, we removed olive oil because it has the highest energy content $(900 \mathrm{kcal} / 100 \mathrm{~g}$ ) whilst being widely considered healthy; this combination pushes the thresholds upwards, because healthiness would imply having more than $900 \mathrm{kcal} / 100 \mathrm{~g}$. The essence of the results is not affected by their inclusion; the figure including olive oil can be found in Figure A3 in Appendix 4, while the thresholds are reported in table A5 in Appendix 4. Table A6 in Appendix 4 reports the coefficients of the multi-level probits used to estimate all thresholds. Results (Table 6) show that traffic-light labels reduce the uncertainty interval, supporting hypothesis 1b: they increases the threshold used to classify products as "good for the environment", whilst lowering the threshold used to classify products as "bad for the environment"; similarly, it lowers the threshold used to classify products as "unhealthy", but has no impact on the threshold for "healthy". Time pressure show the same reduction, but the thresholds are never significantly different from the control group, providing support to hypothesis $2 \mathrm{~b}$. Respondents used actual thresholds that are much higher than those they expect (Figure 3 and Table 6), particularly if olive oil is included in the analysis (Table A3 and Figure A6, Appendix 4). The improvements in accuracy brought by traffic-light labels observed in the previous section is then reflected into a more realistic set of feasible values in the numerical judgement; however, labels are not sufficient to make these thresholds overlap. 


\section{DISCUSSION AND CONCLUSIONS}

This research explores the accuracy of consumers estimates of carbon and kilocalories content of a range of food products commonly found in supermarkets. Results indicate that consumers struggle in estimating both metrics. Traffic-light labelling improves these assessments by improving the ability to rank products by both kilocalories and carbon footprint, with a somewhat smaller impact on the ability to use the metric. Notably, the benefits of traffic-light labelling in terms of reducing time needs during the evaluation is small, an indication that the benefits of traffic-light labels are primarily in terms of learning and metric and mapping knowledge. While labels increase the accuracy of the assessments, they do not make assessments exact. Finally, traffic-light labelling reduces uncertainty over what products are considered unhealthy or bad for the environment, but does not change the threshold used to classify products as healthy. This section discusses the implications of these results.

\subsection{The policy relevance of traffic-light labelling for health and environmental preservation}

Labelling-based food policies in recent years aimed at helping consumers make better choices by making the estimation of the impact of food products on health and the environment easier (Grunert et al. 2010). Traffic-light labels are a good example, and were introduced to improve decision making by (at least partly) allowing consumers to make better and quicker estimate of the nutritional quality of the food they buy (Koenigstorfer et al. 2013; VanEpps et al. 2015). The empirical success of trafficlight labels can be expanded to include carbon footprint (see e.g., Muller et al. 2019; Osman and Thornton 2019). This research shows that traffic-light labels noticeably improve consumer assessments, and are effective in reducing - but not eliminating - estimation biases for both carbon footprint and kilocalories. These improvements are not associated with an increased ability to handle time constraints, but rather a better ability to understand and use a metric; however, time pressure was not enforced, something that might have limited the effectiveness of this manipulation. Notably, when no information is provided, the implicit kilocalories and carbon footprint thresholds consumers use to classify products are considerably different from the actual thresholds they are using. Overall, these results support existing research suggesting that consumers struggle to estimate carbon footprint 
and kilocalories of foods (e.g., Chernev 2011a; Gorissen and Weijters 2016; Liu et al. 2015; Upham et al. 2011). Moreover, the results suggest that consumers in the marketplace struggle to make a environmentally-friendly and healthy basket, and traffic-light labels can lead to significant improvements.

\subsection{Knowledge, familiarity, and the accuracy of the estimated impact of food products}

An important, albeit expected, result is that the benefits of using a traffic-light label are particularly valuable for carbon footprint estimates, whose accuracy (RMSD, metric and mapping knowledge) becomes comparable to that of kilocalories. This result can be explained by the limited familiarity of carbon footprint in the marketplace documented in the literature ${ }^{7}$ (Bleda and Valente 2009; Upham et al. 2011). The notion that familiarity and experience in using the metric are key elements in explaining these results is supported by the fact that kilocalories estimate are in general more accurate, also in the absence of labels; and by the fact that traffic-light labels have no impact on the implicit threshold used to classify products as healthy. The limited availability of information about carbon footprint in the marketplace, and the related low familiarity with it, hinders the ability of consumers to understand the concept of carbon footprint, as well as the ability to identify high or low carbon products in a choice set (see also Panzone et al. 2016a). Notably, both metrics retain a significant degree of inaccuracy even in the presence of traffic-light labels, an indication that familiarity does not fully account for the differences in the results observed in this article. Finally, this exercise presented slightly different traffic light labels for carbon footprint and kilocalories (due to data availability), and the design itself might explain part of these differences; however, the results show that metric and mapping knowledge are very close when traffic lights labels are presented, suggesting this difference in design might not be a main driver of the results.

\footnotetext{
${ }^{7}$ We thank an anonymous reviewer for suggesting this point.
} 


\subsection{Implications of inaccurate assessments on self-monitoring}

Understanding how consumers determine the quality of foods they encounter in the marketplace is key for policy planning. The inability to estimate the kilocalories may lead to the overconsumption (underconsumption) of products that are erroneously considered low (high) in energy content (Chandon and Wansink 2007a; Chernev 2011a); while the inability to estimate the carbon footprint may be associated to the raising threat of climate change (Panzone et al. 2016a). The results in this article only focus on the estimated carbon and kilocalories content of foods, and did not explore the implications of misestimations on quantity purchased; however, if those products for which carbon footprint (kilocalories) is underestimated are purchased much more than those for which carbon footprint (kilocalories) are overestimated are given up, then consumers will emit more GHG (consume more kilocalories) then they expect. At the same time, consumers use "optimistic" thresholds that under-classify items as unhealthy or bad for the environment; traffic-light labels reduce the distance between implicit and actual thresholds, although these remain different.

The inability to correctly estimate the impact of food products on health and the environment is problematic. Self-monitoring ensures behaviour complies to the underlying set of values of the individual (Baumeister 2002; Gino et al. 2011). However, self-monitoring appears particularly problematic for carbon footprint, whose estimates in the control group are very far from the actual values. These results are in line with previous research highlighting that consumers do not fully understand carbon labelling, and struggle in using it correctly (Camilleri et al. 2019; Gorissen and Weijters 2016; Panzone et al. 2016a; Upham et al. 2011); and that similar struggles appear for kilocalories in some contexts (e.g., Chernev 2011a, b; Chernev and Gal 2010; Liu et al. 2015). The notable improvements when a traffic-light label is provided gives an indication that difficulties in self-monitoring can be overcome by providing additional, even simple information (as also found in Andrews et al. 2011; Muller et al. 2019). These findings are particularly relevant for the growing niche of research studying sustainable choices in online environment (Demarque et al. 2015; Muller et al. 2019; Panzone et al. 2018), where consumers make choices without physically seeing products. 
This research does not explore the behavioural part of the self-regulatory process, and questions remains on the extent to which the inability to estimate kilocalories and carbon footprint translates into wrong choices. Some research (e.g., Gigerenzer and Gaissmaier 2011) suggests that inaccuracy is not necessarily problematic: the use of heuristics can still lead to optimal choices because the loss in accuracy due to a reduction in processing time might be small and affecting all options equally. However, research consistently indicate that traffic-light labels change consumer decisions, making them healthier (Andrews et al. 2011; Koenigstorfer et al. 2013; Osman and Thornton 2019; van Herpen and Trijp 2011; VanEpps et al. 2015) and more sustainable (Muller et al. 2019; Osman and Thornton 2019). This literature indicates that improvements in self-monitoring cause consumers to make different decisions, leading to choices that align with personal goals better than initial choices. Improvements in the ability to estimate product quality are largely retained under time pressure, due to the ease of interpretation of the information. The inability of traffic-light labels to help consumers in making near-exact estimate of kilocalories and carbon footprint is a key limitation of this type of intervention: the information conveyed in a traffic-light label is generally simple, and requires consumers to engage in further deliberate thinking to correctly estimate the metric being targeted. Future research could then explore other factors, such as cognitive biases, that can further improve the effectiveness of traffic-light labels as a mean to facilitate quality evaluations.

\subsection{Limitations of the study}

While the research presents a number of robust findings, it also has some limitations. Firstly, this research focused primarily on the evaluation of products by their name, as it would appear on an online shop. In the future, the comparison with a treatment group where all participants have access to all the information typically provided on the sides or back of the packaging would allow testing if simpler information can lead to better assessments than complex information. A second limitation relates to the small sample of products presented to participants. The sample size was determined with a view of having a reasonable survey length, and choosing from a limited pool of products with reliable carbon footprint. This approach limits the generalisability of the findings, and future research 
should explore ways replicate this exercise on a bigger sample of products. Finally, this article did not explore the link between the accuracy of the assessment and consumer decisions, an area of research that should be given increasing attention in future research.

\subsection{Conclusions}

This article presents evidence that consumers struggle to correctly establish the kilocalories and carbon footprint of foods. The presence of traffic-light labelling is a relevant tool to improve the accuracy of assessments, because it facilitates ranking by quality, while its ability to reduce the impact of time pressure is limited. Crucially, the inability to make accurate assessments may limit the ability of consumers to self-monitor and correctly manifest their interest to health and environment in their choices, something that should be tested in future research. The results observed are very similar for both metrics. However, while kilocalories information are presented in labels, the carbon footprint is not, and its addition on food labels could be an effective way to ensure environmental information is incorporated into the decision-making process of consumers. 


\section{REFERENCES}

Andrews, J. Craig, Scot Burton, and Jeremy Kees (2011), "Is Simpler Always Better? Consumer Evaluations of Front-of-Package Nutrition Symbols," Journal of Public Policy \& Marketing, 30 (2), 175-90.

Attari, Shahzeen Z. (2014), "Perceptions of Water Use," Proceedings of the National Academy of Sciences, 111 (14), 5129-34.

Attari, Shahzeen Z., Michael L. DeKay, Cliff I. Davidson, and Wändi Bruine de Bruin (2010), "Public Perceptions of Energy Consumption and Savings," Proceedings of the National Academy of Sciences, 107 (37), 16054-59.

Baumeister, Roy F. (2002), "Yielding to Temptation: Self-Control Failure, Impulsive Purchasing, and Consumer Behavior," Journal of Consumer Research, 28 (4), 670-76.

Berners-Lee, Mike (2011), How Bad Are Bananas?: The Carbon Footprint of Everything: Greystone Books.

Bi, Jian and Daniel M. Ennis (1998), "Sensory Thresholds: Concepts and Methods," Journal of Sensory Studies, 13 (2), 133-48.

Blaylock, James, David Smallwood, Kathleen Kassel, Jay Variyam, and Lorna Aldrich (1999), "Economics, Food Choices, and Nutrition," Food Policy, 24 (2-3), 269-86.

Bleda, Mercedes and Marco Valente (2009), "Graded Eco-Labels: A Demand-Oriented Approach to Reduce Pollution," Technological Forecasting and Social Change, 76 (4), 512-24.

Bowling, Nathan A., Jason L. Huang, Caleb B. Bragg, Steve Khazon, Mengqiao Liu, and Caitlin E. Blackmore (2016), "Who Cares and Who Is Careless? Insufficient Effort Responding as a Reflection of Respondent Personality," Journal of Personality and Social Psychology, 111 (2), 218-29.

Brick, Cameron, David K. Sherman, and Heejung S. Kim (2017), "Green to Be Seen" and "Brown to Keep Down": Visibility Moderates the Effect of Identity on Pro-Environmental Behavior," Journal of Environmental Psychology, 51, 226-38.

Camilleri, Adrian R., Richard P. Larrick, Shajuti Hossain, and Dalia Patino-Echeverri (2019), "Consumers Underestimate the Emissions Associated with Food but Are Aided by Labels," Nature Climate Change, 9 (1), 53-58.

Chandon, Pierre and Brian Wansink (2007a), "The Biasing Health Halos of Fast-Food Restaurant Health Claims: Lower Calorie Estimates and Higher Side-Dish Consumption Intentions," Journal of Consumer Research, 34 (3), 301-14.

--- (2007b), "Is Obesity Caused by Calorie Underestimation? A Psychophysical Model of Meal Size Estimation," Journal of Marketing Research, 44 (1), 84-99.

Chernev, A. and D. Gal (2010), "Categorization Effects in Value Judgments: Averaging Bias in Evaluating Combinations of Vices and Virtues," Journal of Marketing Research, 47 (4), 738-47.

Chernev, Alexander (2011a), "The Dieter's Paradox," Journal of Consumer Psychology, 21 (2), 17883.

--- (2011b), "Semantic Anchoring in Sequential Evaluations of Vices and Virtues," Journal of Consumer Research, 37 (5), 761-74.

Chiou, Wen-Bin, Lien-Te Yeh, and Ming-Hsu Chang (2009), "Effects of Health-Related Claims on the Differential Threshold of Consumers' Sweetness Sensation," Journal of Sensory Studies, 24 (4), 621-33.

Cornelissen, Gert, Mario Pandelaere, Luk Warlop, and Siegfried Dewitte (2008), "Positive Cueing: Promoting Sustainable Consumer Behavior by Cueing Common Environmental Behaviors as Environmental," International Journal of Research in Marketing, 25 (1), 46-55.

Curran, Paul G. (2016), "Methods for the Detection of Carelessly Invalid Responses in Survey Data," Journal of Experimental Social Psychology, 66, 4-19.

Demarque, Christophe, Laetitia Charalambides, Denis J. Hilton, and Laurent Waroquier (2015), "Nudging Sustainable Consumption: The Use of Descriptive Norms to Promote a Minority Behavior in a Realistic Online Shopping Environment," Journal of Environmental Psychology, 43, 166-74. 
Drewnowski, Adam, Colin D Rehm, Agnes Martin, Eric O Verger, Marc Voinnesson, and Philippe Imbert (2015), "Energy and Nutrient Density of Foods in Relation to Their Carbon Footprint," The American Journal of Clinical Nutrition, 101 (1), 184-91.

Epley, Nicholas and Thomas Gilovich (2006), "The Anchoring-and-Adjustment Heuristic: Why the Adjustments Are Insufficient," Psychological Science, 17 (4), 311-18.

European Commission (2009), "Europeans' Attitudes Towards the Issue of Sustainable Consumption and Production," in Flash Eurobarometer: European Commission, Directorate-General for the Environment.

Faulkner, G. P., L. K. Pourshahidi, J. M. W. Wallace, M. A. Kerr, T. A. McCaffrey, and M. B. E. Livingstone (2014), "Perceived 'Healthiness' of Foods Can Influence Consumers' Estimations of Energy Density and Appropriate Portion Size," International Journal of Obesity, 38 (1), 106-12.

Gigerenzer, Gerd and Wolfgang Gaissmaier (2011), "Heuristic Decision Making," Annual Review of Psychology, 62 (1), 451-82.

Gino, Francesca, Maurice E. Schweitzer, Nicole L. Mead, and Dan Ariely (2011), "Unable to Resist Temptation: How Self-Control Depletion Promotes Unethical Behavior," Organizational Behavior and Human Decision Processes, 115 (2), 191-203.

Gorissen, Karen and Bert Weijters (2016), "The Negative Footprint Illusion: Perceptual Bias in Sustainable Food Consumption," Journal of Environmental Psychology, 45, 50-65.

Grunert, Klaus G., Josephine M. Wills, and Laura Fernández-Celemín (2010), "Nutrition Knowledge, and Use and Understanding of Nutrition Information on Food Labels among Consumers in the Uk," Appetite, 55 (2), 177-89.

Hartmann, Christina and Michael Siegrist (2017), "Consumer Perception and Behaviour Regarding Sustainable Protein Consumption: A Systematic Review," Trends in Food Science \& Technology, 61, 11-25.

Hertwig, Ralph, Thorsten Pachur, and Stephanie Kurzenhäuser (2005), "Judgments of Risk Frequencies: Tests of Possible Cognitive Mechanisms," Journal of Experimental Psychology: Learning, Memory, and Cognition, 31 (4), 621-42.

Huang, Jason L., Paul G. Curran, Jessica Keeney, Elizabeth M. Poposki, and Richard P. DeShon (2012), "Detecting and Deterring Insufficient Effort Responding to Surveys," Journal of Business and Psychology, 27 (1), 99-114.

Knoblauch, Kenneth and Laurence T. Maloney (2012), Modeling Psychophysical Data in R, New York, NY: New York, NY : Springer.

Koenigstorfer, Joerg, Andrea Groeppel-Klein, and Friederike Kamm (2013), "Healthful Food Decision Making in Response to Traffic Light Color-Coded Nutrition Labeling," Journal of Public Policy \& Marketing, 33 (1), 65-77.

Lamberts, Koen (1995), "Categorization under Time Pressure," Journal of Experimental Psychology: General, 124 (2), 161-80.

Lawless, Harry T. and Hildegarde Heymann (2010), "Measurement of Sensory Thresholds," in Sensory Evaluation of Food: Principles and Practices, New York, NY: Springer New York, 125-47. Lee, Wan-chen Jenny, Mitsuru Shimizu, Kevin M. Kniffin, and Brian Wansink (2013), "You Taste What You See: Do Organic Labels Bias Taste Perceptions?," Food Quality and Preference, 29 (1), 33-39.

Liu, Peggy J, James R Bettman, Arianna R Uhalde, and Peter A Ubel (2015), "'How Many Calories Are in My Burrito?' Improving Consumers' Understanding of Energy (Calorie) Range Information," Public Health Nutrition, 18 (01), 15-24.

Logvinenko, Alexander D., Yuri N. Tyurin, and Martin Sawey (2012), "A Test for Psychometric Function Shift," Behavior Research Methods, 44 (2), 503-15.

Macdiarmid, Jennie I, Janet Kyle, Graham W Horgan, Jennifer Loe, Claire Fyfe, Alexandra Johnstone, and Geraldine McNeill (2012), "Sustainable Diets for the Future: Can We Contribute to Reducing Greenhouse Gas Emissions by Eating a Healthy Diet?," The American Journal of Clinical Nutrition, 96 (3), 632-39. 
Meade, Adam W. and S. Bartholomew Craig (2012), "Identifying Careless Responses in Survey Data," Psychological Methods, 17 (3), 437-55.

Mochon, Daniel and Shane Frederick (2013), "Anchoring in Sequential Judgments," Organizational Behavior and Human Decision Processes, 122 (1), 69-79.

Moorman, Christine (1996), "A Quasi Experiment to Assess the Consumer and Informational Determinants of Nutrition Information Processing Activities: The Case of the Nutrition Labeling and Education Act," Journal of Public Policy \& Marketing, 15 (1), 28-44.

Morris, Elizabeth (1993), "Nutrition-Labelling Developments," British Food Journal, 95 (5), 12-15. Muller, Laurent, Anne Lacroix, and Bernard Ruffieux (2019), "Environmental Labelling and Consumption Changes: A Food Choice Experiment," Environmental and Resource Economics, 73 (3), 871-97.

Mussweiler, Thomas and Fritz Strack (1999), "Hypothesis-Consistent Testing and Semantic Priming in the Anchoring Paradigm: A Selective Accessibility Model," Journal of Experimental Social Psychology, 35 (2), 136-64.

--- (2000a), "Numeric Judgments under Uncertainty: The Role of Knowledge in Anchoring," Journal of Experimental Social Psychology, 36 (5), 495-518.

--- (2000b), "The Use of Category and Exemplar Knowledge in the Solution of Anchoring Tasks," Journal of Personality and Social Psychology, 78 (6), 1038-52.

Osman, Magda and Katie Thornton (2019), "Traffic Light Labelling of Meals to Promote Sustainable Consumption and Healthy Eating," Appetite, 138, 60-71.

Panzone, Luca A., Fred Lemke, and Henry L. Petersen (2016a), "Biases in Consumers' Assessment of Environmental Damage in Food Chains and How Investments in Reputation Can Help," Technological Forecasting and Social Change, 111, 327-37.

Panzone, Luca A., Alistair Ulph, Daniel John Zizzo, Denis Hilton, and Adrian Clear (2018), "The Impact of Environmental Recall and Carbon Taxation on the Carbon Footprint of Supermarket Shopping," Journal of Environmental Economics and Management.

Panzone, Luca, Denis Hilton, Laura Sale, and Doron Cohen (2016b), "Socio-Demographics, Implicit Attitudes, Explicit Attitudes, and Sustainable Consumption in Supermarket Shopping," Journal of Economic Psychology, 55, 77-95.

Parmenter, Kathryn and Jane Wardle (1999), "Development of a General Nutrition Knowledge Questionnaire for Adults," European Journal of Clinical Nutrition, 53 (4), 298-308.

Pieters, Rik and Luk Warlop (1999), "Visual Attention During Brand Choice: The Impact of Time Pressure and Task Motivation," International Journal of Research in Marketing, 16 (1), 1-16.

Poore, J. and T. Nemecek (2018), "Reducing Food's Environmental Impacts through Producers and Consumers," Science, 360 (6392), 987-92.

Prothero, Andrea, Susan Dobscha, Jim Freund, William E. Kilbourne, Michael G. Luchs, Lucie K. Ozanne, and John Thøgersen (2011), "Sustainable Consumption: Opportunities for Consumer Research and Public Policy," Journal of Public Policy \& Marketing, 30 (1), 31-38.

Reutskaja, Elena, Rosemarie Nagel, Colin F. Camerer, and Antonio Rangel (2011), "Search Dynamics in Consumer Choice under Time Pressure: An Eye-Tracking Study," American Economic Review, 101 (2), 900-26.

Scheibehenne, Benjamin (2019), "The Psychophysics of Number Integration: Evidence from the Lab and from the Field," Decision, 6 (1), 61-76.

Schuldt, Jonathon P and Norbert Schwarz (2010), "The" Organic" Path to Obesity? Organic Claims Influence Calorie Judgments and Exercise Recommendations," Judgment and decision making, 5 (3), 144-50.

Shah, Avni M., James R. Bettman, Peter A. Ubel, Punam Anand Keller, and Julie A. Edell (2014), "Surcharges Plus Unhealthy Labels Reduce Demand for Unhealthy Menu Items," Journal of Marketing Research, 51 (6), 773-89. 
Shi, Jing, Vivianne H. M. Visschers, Noëmi Bumann, and Michael Siegrist (2018), "Consumers' Climate-Impact Estimations of Different Food Products," Journal of Cleaner Production, 172, 164653.

Simmons, Joseph P., Robyn A. LeBoeuf, and Leif D. Nelson (2010), "The Effect of Accuracy Motivation on Anchoring and Adjustment: Do People Adjust from Provided Anchors?," Journal of Personality and Social Psychology, 99 (6), 917-32.

Smith, Andrew R. and Paul D. Windschitl (2015), "Resisting Anchoring Effects: The Roles of Metric and Mapping Knowledge," Memory \& Cognition, 43 (7), 1071-84.

Springmann, Marco, H. Charles J. Godfray, Mike Rayner, and Peter Scarborough (2016), "Analysis and Valuation of the Health and Climate Change Cobenefits of Dietary Change," Proceedings of the National Academy of Sciences, 113 (15), 4146-51.

Steg, Linda and Charles Vlek (2009), "Encouraging Pro-Environmental Behaviour: An Integrative Review and Research Agenda," Journal of Environmental Psychology, 29 (3), 309-17.

Suri, Rajneesh and Kent B. Monroe (2003), "The Effects of Time Constraints on Consumers' Judgments of Prices and Products," Journal of Consumer Research, 30 (1), 92-104.

Tversky, Amos and Daniel Kahneman (1974), "Judgment under Uncertainty: Heuristics and Biases," Science, 185 (4157), 1124-31.

Ülkümen, Gülden, Manoj Thomas, and Vicki G. Morwitz (2008), "Will I Spend More in 12 Months or a Year? The Effect of Ease of Estimation and Confidence on Budget Estimates," Journal of Consumer Research, 35 (2), 245-56.

Upham, Paul, Leonie Dendler, and Mercedes Bleda (2011), "Carbon Labelling of Grocery Products: Public Perceptions and Potential Emissions Reductions," Journal of Cleaner Production, 19 (4), 34855.

van Herpen, Erica and Hans C. M. van Trijp (2011), "Front-of-Pack Nutrition Labels. Their Effect on Attention and Choices When Consumers Have Varying Goals and Time Constraints," Appetite, 57 (1), 148-60.

Vanclay, JeromeK, John Shortiss, Scott Aulsebrook, AngusM Gillespie, BenC Howell, Rhoda Johanni, MichaelJ Maher, KellyM Mitchell, MarkD Stewart, and Jim Yates (2011), "Customer Response to Carbon Labelling of Groceries," Journal of Consumer Policy, 34 (1), 153-60.

VanEpps, Eric M., Julie S. Downs, and George Loewenstein (2015), "Calorie Label Formats: Using Numbers or Traffic Lights to Reduce Lunch Calories," Journal of Public Policy \& Marketing.

Wang, Hua (1997), "Treatment of "Don't-Know" Responses in Contingent Valuation Surveys: A Random Valuation Model," Journal of Environmental Economics and Management, 32 (2), 219-32. Wansink, Brian and Pierre Chandon (2006), "Can "Low-Fat" Nutrition Labels Lead to Obesity?," Journal of Marketing Research, 43 (4), 605-17.

Whitmarsh, Lorraine, Gill Seyfang, and Saffron O’Neill (2011), "Public Engagement with Carbon and Climate Change: To What Extent Is the Public 'Carbon Capable'?," Global Environmental Change, 21 (1), 56-65. 


\section{FIGURES}

Figure 1: Examples of labels used in the "labelling" condition

a) Carbon label - butter

\begin{tabular}{l|l|l|l|l|}
$\begin{array}{l}\text { Carbon emissions } \\
\text { from sector }\end{array}$ & & & & \\
& $\begin{array}{c}\text { Raw material } \\
\text { production }\end{array}$ & $\begin{array}{c}\text { Manufacture } \\
\text { /processing }\end{array}$ & $\begin{array}{c}\text { Logistics } \\
\text { /distribution }\end{array}$ & Retail \\
\hline English Unsalted Butter & MEDIUM & HIGH & LOW & HIGH \\
\hline English Salted Butter & & HIGH & LOW & HIGH \\
\hline
\end{tabular}

b) Carbon label - mushrooms

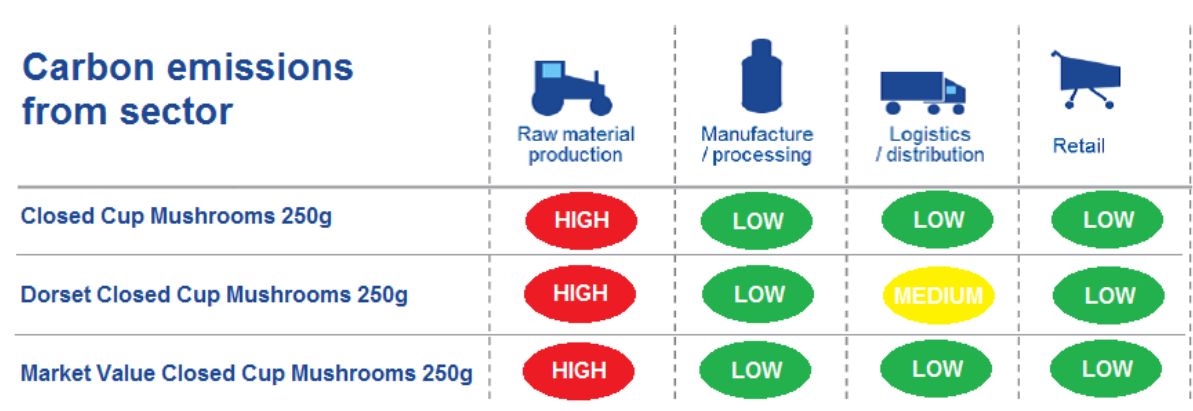

c) Nutrition label - butter

\begin{tabular}{l|c|c|c} 
Nutrient content $\mathbf{( 1 0 0} \mathbf{~ g})$ & $\begin{array}{l}\text { Carbohydrates } \\
\text { (incl. sugars) }\end{array}$ & Fats & Salt \\
\hline English Unsalted Butter & $1.1 . \mathrm{g}$ & $\mathbf{8 2 . 2 \mathrm { g }}$ & $\mathbf{0 g}$ \\
\hline English Salted Butter & $0.8 \mathrm{~g}$ & $\mathbf{8 1 . 3 \mathrm { g }}$ & $\mathbf{0 . 1 \mathrm { g }}$
\end{tabular}

d) Nutrition label - mushrooms

\begin{tabular}{l|c|c|c} 
Nutrient content (100 g) & $\begin{array}{c}\text { Carbohydrates } \\
\text { (incl. sugars) }\end{array}$ & Fats & Salt \\
\hline Closed Cup Mushrooms 250g & $0.4 \mathrm{~g}$ & $0.5 \mathrm{~g}$ & $0.1 \mathrm{~g}$ \\
\hline Dorset Closed Cup Mushrooms 250g & $0.4 \mathrm{~g}$ & $0.5 \mathrm{~g}$ & $0.1 \mathrm{~g}$ \\
\hline Market Value Closed Cup Mushrooms 250g & $0.4 \mathrm{~g}$ & $0.5 \mathrm{~g}$ & $0.1 \mathrm{~g}$ \\
\hline
\end{tabular}


Figure 2: True and expected relation between carbon footprint and kilocalories

a) Carbon footprint
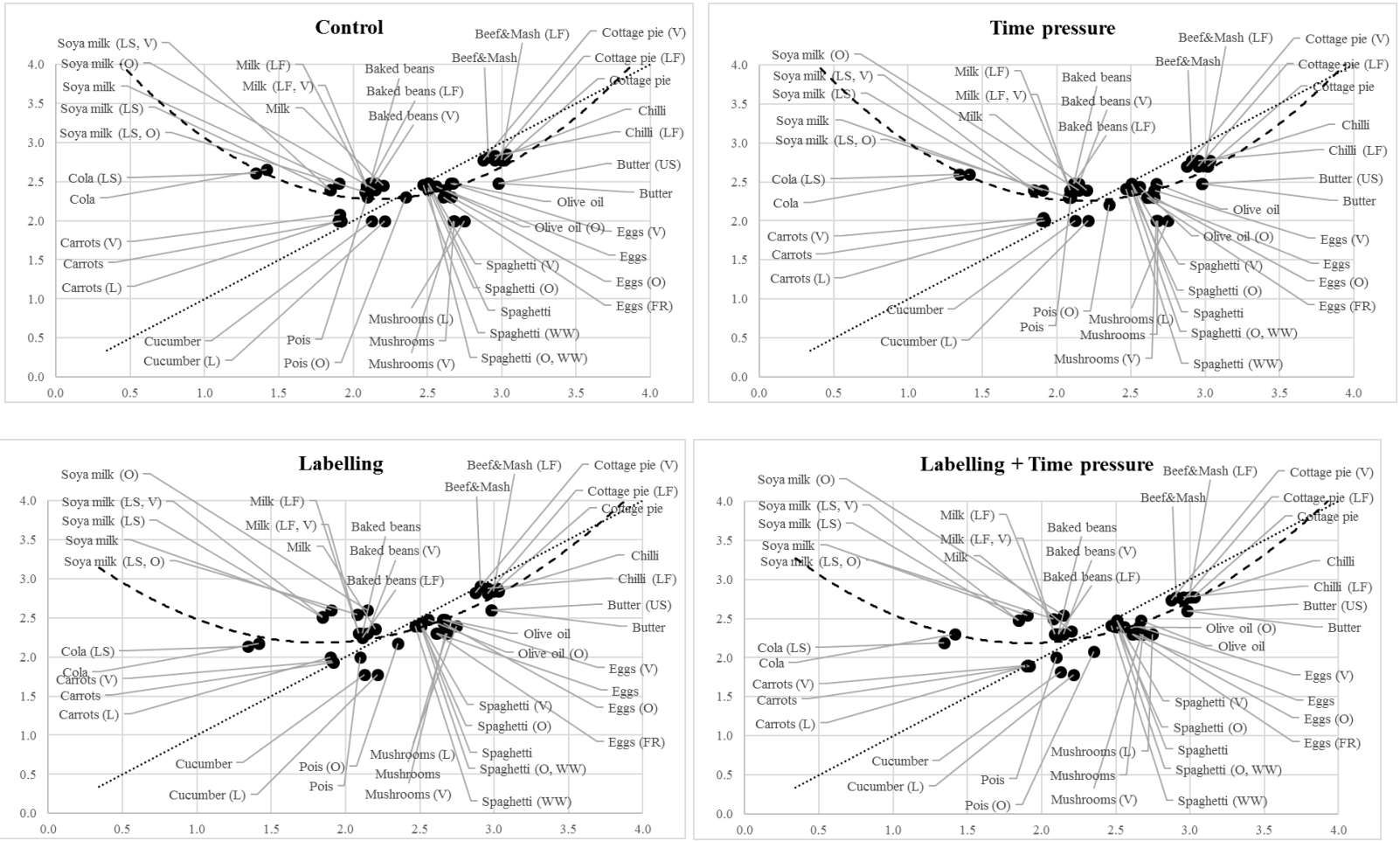

b) Kilocalories
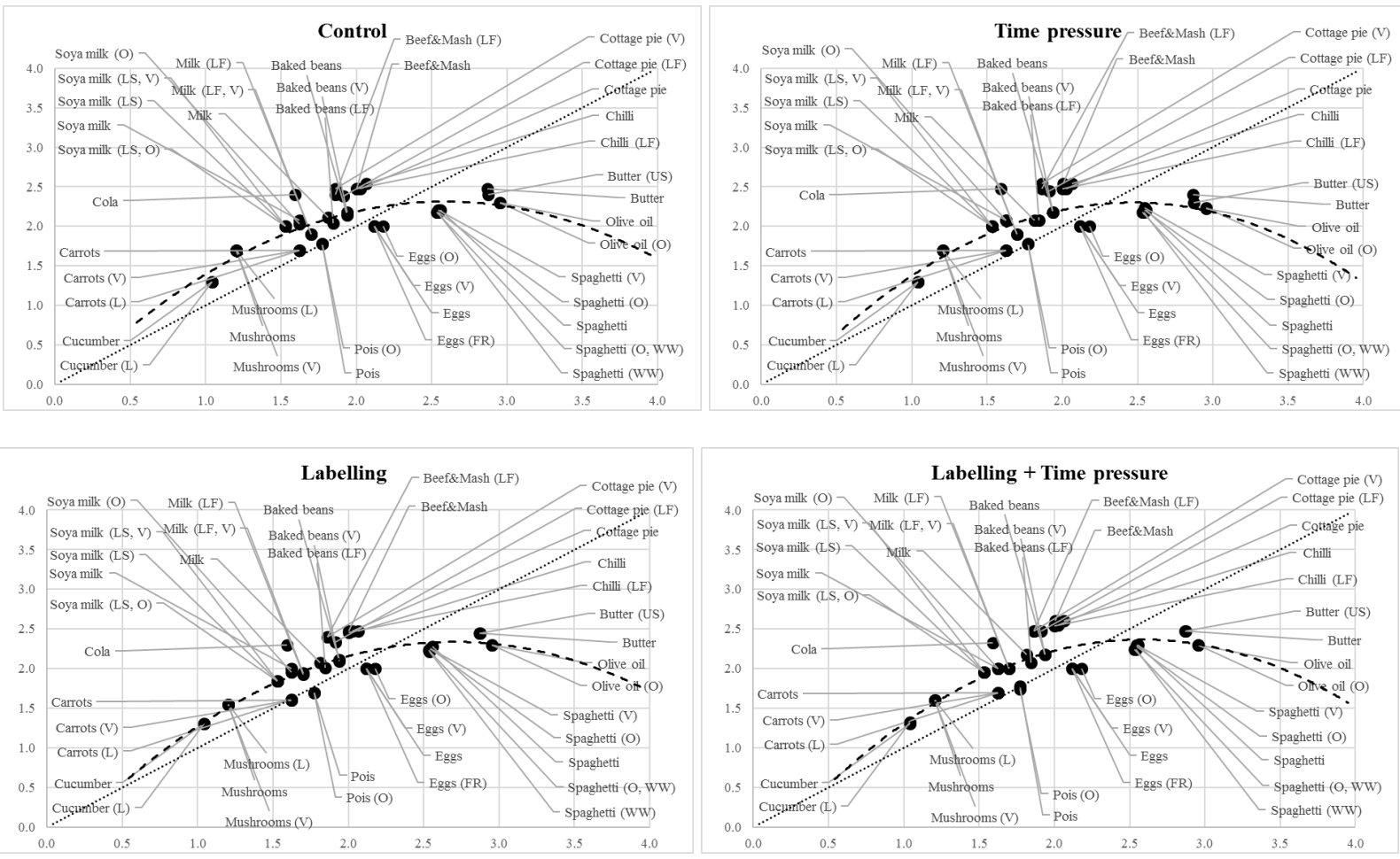

Note: Axes are in logarithmic form, base 10. In figure b), diet cola is not presented as its real kilocalories content is zero. The dotted line refers to perfect assessments; while the dashed line is the quadratic line best fitting the data. 
Figure 3: Graphical representation of the estimated thresholds, by metric and treatment

a) Carbon footprint

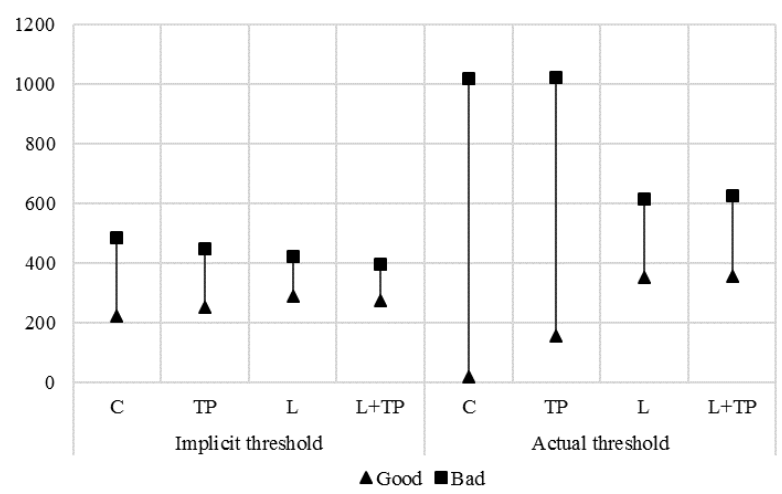

b) Kilocalories (excl. olive oil)

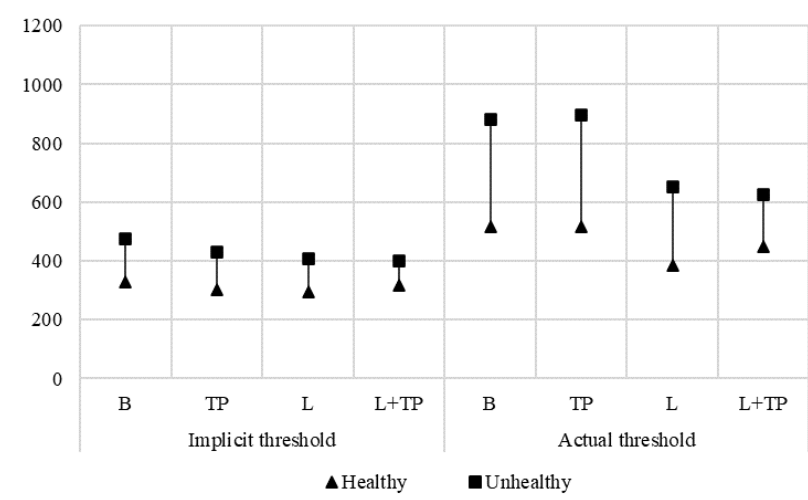

Acronyms are as follows: $\mathrm{C}=\mathrm{Control}$; $\mathrm{TP}=$ Time pressure; $\mathrm{L}=$ Labelling; $\mathrm{L}+\mathrm{TP}=$ joint Time pressure and Labelling. 


\section{TABLES}

Table 1: Summary quality claims on product labels in the sample

\begin{tabular}{l|c|c}
\hline Claim & Frequency & Claim type \\
\hline No claim & 15 & Baseline \\
English geographical reference & 5 & Local \\
Free-range & 1 & Free-range \\
Light choice/low-fat & 5 & Health \\
Low salt & 1 & Health \\
Low sugar & 3 & Health \\
Organic & 7 & Organic \\
Value & 8 & Value \\
Whole wheat & 2 & Health \\
\hline
\end{tabular}

Table 2: Summary characteristics of the full sample

\begin{tabular}{|c|c|c|c|c|c|c|c|c|c|c|c|}
\hline \multirow[b]{2}{*}{ Variable } & \multicolumn{2}{|l|}{ All } & \multicolumn{2}{|l|}{$\mathbf{C}$} & \multicolumn{2}{|l|}{ TP } & \multicolumn{2}{|l|}{$\mathbf{L}$} & \multicolumn{2}{|l|}{$\mathbf{L}+\mathbf{T P}$} & \multirow[b]{2}{*}{$\begin{array}{c}K-W \text { test } \\
\chi^{2}\end{array}$} \\
\hline & Mean & S.D. & Mean & S.D. & Mean & S.D. & Mean & S.D. & Mean & S.D. & \\
\hline Age & 48.40 & 17.33 & 48.14 & 17.67 & 48.34 & 17.34 & 48.19 & 17.06 & 49.02 & 17.30 & 0.574 \\
\hline Male & 0.47 & 0.50 & 0.44 & 0.50 & 0.47 & 0.50 & 0.49 & 0.50 & 0.49 & 0.50 & 2.488 \\
\hline Environmental attitudes & 4.10 & 0.87 & 4.04 & 0.92 & 4.14 & 0.81 & 4.07 & 0.90 & 4.13 & 0.86 & 1.872 \\
\hline Health attitudes & 3.84 & 1.01 & 3.82 & 1.01 & 3.78 & 1.02 & 3.85 & 1.00 & 3.94 & 1.03 & 6.647 \\
\hline Environmental identity & 3.73 & 1.02 & 3.72 & 1.05 & 3.78 & 0.99 & 3.68 & 1.03 & 3.73 & 1.04 & 2.022 \\
\hline Health identity & 3.35 & 1.07 & 3.35 & 1.07 & 3.30 & 1.09 & 3.37 & 1.05 & 3.37 & 1.07 & 1.210 \\
\hline Footprint knowledge & 0.80 & 0.70 & 0.80 & 0.72 & 0.79 & 0.69 & 0.81 & 0.69 & 0.78 & 0.71 & 0.311 \\
\hline Kilocalories knowledge & 0.77 & 0.72 & 0.73 & 0.72 & 0.73 & 0.70 & 0.81 & 0.72 & 0.80 & 0.72 & 4.469 \\
\hline Frequency - environment & 0.85 & 1.05 & 0.83 & 1.03 & 0.85 & 1.08 & 0.87 & 1.06 & 0.88 & 1.06 & 0.647 \\
\hline Frequency - health & 1.31 & 1.25 & 1.25 & 1.19 & 1.35 & 1.32 & 1.32 & 1.26 & 1.31 & 1.24 & 0.522 \\
\hline Survey time (min) & 33.00 & 94.76 & 34.68 & 59.73 & 23.96 & 95.26 & 43.07 & 128.87 & 30.09 & 76.94 & $147.05 * *$ \\
\hline $\mathrm{CO}_{2}$ evaluation time & 14.32 & 83.52 & 13.00 & 12.59 & 9.10 & 18.75 & 24.27 & 164.84 & 9.87 & 8.66 & $167.47 * *$ \\
\hline Kcal evaluation time & 16.32 & 64.89 & 19.68 & 59.18 & 13.87 & 101.75 & 21.99 & 54.77 & 10.64 & 10.83 & $226.99 * *$ \\
\hline
\end{tabular}

Significance is as follows: ${ }^{*} \mathrm{p}<0.05$; ${ }^{*} \mathrm{p}<0.01$. Note: means refer to the sample included in the analyses below. Acronyms are as follows: $\mathrm{C}=$ Control; $\mathrm{TP}=$ Time pressure; $\mathrm{L}=$ Labelling; $\mathrm{L}+\mathrm{TP}=$ joint Time pressure and Labelling; $\mathrm{K}-\mathrm{W}=$ Kruskal-Wallis chi ${ }^{2}$, testing differences between treatments.

Table 3: Estimated metric and mapping knowledge in the sample

\begin{tabular}{l|l|l|cccc|c}
\hline & & $\mathbf{C}$ & $\mathbf{T P}$ & $\mathbf{L}$ & $\mathbf{L}+\mathbf{T P}$ & $\mathbf{K}-\mathbf{W}$ test $\chi^{2}$ \\
\hline Carbon footprint & RSMD & Mean & 331.70 & 340.80 & $290.68^{* *}$ & $308.76^{* *}$ & $87.32^{* *}$ \\
& & SD & 73.73 & 73.68 & 79.46 & 80.77 & \\
\cline { 2 - 8 } & OME & Mean & 0.46 & 0.47 & $0.41^{* *}$ & $0.44^{* *}$ & $68.82^{* *}$ \\
& (metric knowledge) & SD & 0.22 & 0.23 & 0.24 & 0.25 & \\
\cline { 2 - 9 } & Spearman $\rho$ & Mean & 0.41 & $0.34 *$ & $0.59 * *$ & $0.54^{* *}$ & $235.25 * *$ \\
& (mapping knowledge) & SD & 0.28 & 0.30 & 0.23 & 0.26 & \\
\hline
\end{tabular}




\begin{tabular}{|c|c|c|c|c|c|c|c|}
\hline & Observations & & 296 & 318 & 325 & 279 & \\
\hline \multirow{7}{*}{ Kilocalories } & \multirow[t]{2}{*}{ RSMD } & Mean & 267.51 & 275.38 & $249.84 * *$ & 265.28 & \multirow[t]{2}{*}{$24.52 * *$} \\
\hline & & SD & 82.86 & 69.52 & 76.70 & 72.63 & \\
\hline & \multirow{2}{*}{$\begin{array}{l}\text { OME } \\
\text { (metric knowledge) }\end{array}$} & Mean & 0.49 & $0.51 * *$ & $0.45 * *$ & 0.48 & \multirow[t]{2}{*}{$33.52 * *$} \\
\hline & & SD & 0.16 & 0.15 & 0.16 & 0.15 & \\
\hline & \multirow{2}{*}{$\begin{array}{l}\text { Spearman } \rho \\
\text { (mapping knowledge) }\end{array}$} & Mean & 0.47 & $0.41 * *$ & $0.54 * *$ & 0.49 & \multirow[t]{2}{*}{$55.42 * *$} \\
\hline & & SD & 0.25 & 0.25 & 0.24 & 0.25 & \\
\hline & Observations & & 350 & 371 & 361 & 321 & \\
\hline
\end{tabular}

Note: OME measures metric knowledge, while Spearman correlation measures mapping knowledge. RSMD refers to Root-Square-Mean-Deviation. Exact assessments correspond to $\mathrm{RMSD}=0, \mathrm{OME}=0$, a Spearman $\rho=1$. Significance is as follows: $* \mathrm{p}<0.05 ; * * \mathrm{p}<0.01$. Differences between treatments refer to a Kruskal-Wallis test comparing the control group and the specific treatment. Acronyms are as follows: $\mathrm{C}=\mathrm{Control} ; \mathrm{TP}=$ Time pressure; $\mathrm{L}=\mathrm{Labelling} ; \mathrm{L}+\mathrm{TP}=$ joint Time pressure and Labelling; K-W = Kruskal-Wallis.

Table 4: ANOVA results, univariate $F$ values

\begin{tabular}{|c|c|c|c|c|c|c|c|}
\hline & & \multicolumn{2}{|c|}{ RSMD } & \multicolumn{2}{|c|}{ OME } & \multicolumn{2}{|c|}{ Spearman $\rho$} \\
\hline & & $\mathrm{CO}_{2}$ & Kilocalories & $\mathrm{CO}_{2}$ & Kilocalories & $\mathrm{CO}_{2}$ & Kilocalories \\
\hline Model & & $27.45 * *$ & $7.29 * *$ & $4.14 * *$ & $9.50 * *$ & $86.21 * *$ & $17.97 * *$ \\
\hline Main Effects & TP & $9.47 * *$ & $8.31 * *$ & 2.31 & $11.35 * *$ & $12.41 * *$ & $16.03 * *$ \\
\hline & $\mathbf{L}$ & $68.41 * *$ & $11.80 * *$ & $9.35 * *$ & $15.90 * *$ & $239.26^{* *}$ & $35.53 * *$ \\
\hline Interaction Effects & $\mathrm{TP} \times \mathrm{L}$ & 1.03 & 0.88 & 0.17 & 0.01 & 0.02 & 0.11 \\
\hline Observations & & 1,218 & 1,403 & 1,218 & 1,403 & 1,218 & 1,403 \\
\hline $\mathbf{R}^{2}$ & & 0.06 & 0.02 & 0.01 & 0.02 & 0.18 & 0.04 \\
\hline Adjusted $\mathbf{R}^{2}$ & & 0.06 & 0.01 & 0.01 & 0.02 & 0.17 & 0.04 \\
\hline
\end{tabular}

Note: Significance is as follows: ${ }^{*} \mathrm{p}<0.05 ;{ }^{*} \mathrm{p}<0.01$.

Table 5: Regression of estimated value against actual values

\begin{tabular}{|c|c|c|c|c|c|c|c|c|}
\hline & \multicolumn{4}{|c|}{ Carbon footprint } & \multicolumn{4}{|c|}{ Kilocalories } \\
\hline & Coef. & S.E. & Coef. & S.E. & Coef. & S.E. & Coef. & S.E. \\
\hline Intercept & $-0.1899 * *$ & 0.0213 & $-0.1721 * *$ & 0.0225 & $0.1502 * *$ & 0.0154 & $0.1482 * *$ & 0.0153 \\
\hline $\log _{10}\left(y^{*}\right)$ & $0.2638 * *$ & 0.0134 & $0.2762 * *$ & 0.0151 & $0.3635^{* *}$ & 0.0134 & $0.3884 * *$ & 0.0143 \\
\hline$\left[\log _{10}\left(y^{*}\right)\right]^{2}$ & $0.5277 * *$ & 0.0272 & $0.5353 * *$ & 0.0303 & $-0.0372 * *$ & 0.0107 & $-0.0454 * *$ & 0.0116 \\
\hline TP & -0.0196 & 0.0301 & -0.0187 & 0.0297 & -0.0054 & 0.0223 & -0.0046 & 0.0221 \\
\hline $\mathbf{L}$ & -0.0046 & 0.0297 & -0.0040 & 0.0290 & -0.0384 & 0.0213 & -0.0389 & 0.0209 \\
\hline TP $\times \mathbf{L}$ & -0.0337 & 0.0310 & -0.0303 & 0.0310 & 0.0103 & 0.0215 & 0.0103 & 0.0214 \\
\hline$T P \times \log 10\left(y^{*}\right)$ & $-0.0441 *$ & 0.0192 & $-0.0432 *$ & 0.0191 & $-0.0605 * *$ & 0.0184 & $-0.0610 * *$ & 0.0180 \\
\hline$L \times \log 10\left(y^{*}\right)$ & $0.1856^{* *}$ & 0.0193 & $0.1862 * *$ & 0.0193 & $0.0619 * *$ & 0.0189 & $0.0602 * *$ & 0.0188 \\
\hline TP $x$ L $\times \log 10\left(y^{*}\right)$ & $0.1280 * *$ & 0.0213 & $0.1306^{* *}$ & 0.0210 & 0.0204 & 0.0196 & 0.0205 & 0.0190 \\
\hline$T P x\left[\log 10\left(y^{*}\right)\right]^{2}$ & 0.0193 & 0.0359 & 0.0198 & 0.0358 & 0.0049 & 0.0149 & 0.0064 & 0.0146 \\
\hline$L x\left[\log 10\left(y^{*}\right)\right]^{2}$ & $-0.0693 *$ & 0.0341 & $-0.0684^{*}$ & 0.0342 & -0.0123 & 0.0148 & -0.0119 & 0.0146 \\
\hline TP $x$ L $x\left[\log 10\left(y^{*}\right)\right]^{2}$ & -0.0490 & 0.0357 & -0.0490 & 0.0358 & -0.0226 & 0.0149 & -0.0212 & 0.0147 \\
\hline Age & & & -0.0161 & 0.0111 & & & -0.0007 & 0.0081 \\
\hline Male & & & $-0.0485^{*}$ & 0.0218 & & & $-0.0206^{* *}$ & 0.0076 \\
\hline Attitudes & & & 0.0154 & 0.0122 & & & 0.0088 & 0.0108 \\
\hline Identity & & & 0.0010 & 0.0131 & & & -0.0142 & 0.0110 \\
\hline Knowledge & & & $0.0379 * *$ & 0.0112 & & & $0.0261 * *$ & 0.0079 \\
\hline
\end{tabular}




\begin{tabular}{|c|c|c|c|c|c|c|}
\hline Frequency of purchase & & 0.0032 & 0.0109 & & 0.0029 & 0.0082 \\
\hline $\operatorname{Age} x \log 10\left(y^{*}\right)$ & & $-0.0175^{*}$ & 0.0073 & & -0.0132 & 0.0070 \\
\hline Male $x \log 10\left(y^{*}\right)$ & & $-0.0310^{*}$ & 0.0144 & & $-0.0580^{* *}$ & 0.0132 \\
\hline Attitudes $x \log 10\left(y^{*}\right)$ & & 0.0046 & 0.0078 & & 0.0040 & 0.0083 \\
\hline Identity $x \log 10\left(y^{*}\right)$ & & -0.0004 & 0.0093 & & -0.0115 & 0.0085 \\
\hline Knowledge $x \log 10\left(y^{*}\right)$ & & $0.0166^{*}$ & 0.0072 & & $0.0422 * *$ & 0.0067 \\
\hline Frequency $x \log 10\left(y^{*}\right)$ & & -0.0044 & 0.0070 & & $0.0196 * *$ & 0.0068 \\
\hline Age $x\left[\log 10\left(y^{*}\right)\right]^{2}$ & & 0.0088 & 0.0123 & & $0.0179 * *$ & 0.0053 \\
\hline Male $x\left[\log 10\left(y^{*}\right)\right]^{2}$ & & -0.0185 & 0.0234 & & 0.0178 & 0.0102 \\
\hline Attitudes $x\left[\log 10\left(y^{*}\right)\right]^{2}$ & & 0.0034 & 0.0135 & & $-0.0201 * *$ & 0.0063 \\
\hline Identity $x\left[\log 10\left(y^{*}\right)\right]^{2}$ & & 0.0061 & 0.0150 & & $0.0267 * *$ & 0.0069 \\
\hline Knowledge $x\left[\log 10\left(y^{*}\right)\right]^{2}$ & & 0.0051 & 0.0116 & & -0.0065 & 0.0052 \\
\hline Frequency $x\left[\log 10\left(y^{*}\right)\right]^{2}$ & & -0.0044 & 0.0120 & & $-0.0152 * *$ & 0.0053 \\
\hline \multicolumn{7}{|c|}{ Interaction term - Wald chi ${ }^{2}(1)$} \\
\hline$T P=T P \times L$ & 0.21 & 0.14 & & 0.51 & 0.47 & \\
\hline$L=\mathbf{T P} \times \mathbf{L}$ & 0.91 & 0.75 & & $5.41^{*}$ & $5.56^{*}$ & \\
\hline $\log 10\left(y^{*}\right): T P=T P \times L$ & $64.33^{* *}$ & $67.07^{* *}$ & & $17.92 * *$ & $20.04 * *$ & \\
\hline $\log 10\left(y^{*}\right): L=T P \times L$ & $7.11^{* *}$ & $6.68^{* *}$ & & $4.50 *$ & $4.37^{*}$ & \\
\hline$\left[\log 10\left(y^{*}\right)\right]^{2}: T P=T P \times L$ & $4.31^{*}$ & $4.36^{*}$ & & 3.51 & 3.65 & \\
\hline$\left[\log 10\left(y^{*}\right)\right]^{2}: L=T P \times L$ & 0.43 & 0.39 & & 0.50 & 0.42 & \\
\hline Observations & 50,248 & 50,248 & & 57,653 & 57,653 & \\
\hline Respondents & 1218 & 1218 & & 1403 & 1403 & \\
\hline Model test - Wald chi²(11) & $3260.19^{* *}$ & $3765.46^{* *}$ & & $3128.30 * *$ & $3608.01^{* *}$ & \\
\hline Log pseudolikelihood & -20865.57 & -20840.53 & & -33345.4500 & -33273.72 & \\
\hline
\end{tabular}

Note: Significance is as follows: ${ }^{*} \mathrm{p}<0.05 ;{ }^{*} \mathrm{p}<0.01$. Acronyms are as follows: $\mathrm{TP}=$ Time pressure; $\mathrm{L}=\mathrm{Labelling}$; $\mathrm{L}+\mathrm{TP}$ $=$ joint Time pressure and Labelling. Health attitudes, identity, and knowledge are used for kilocalories; environmental attitudes, identity, and knowledge are used for carbon footprint. Age, attitudes, identity and knowledge have been standardised before entering the regression, so that the intercept captures the average bias at the mean of these variables.

Table 6: Estimated thresholds from the multi-level probit analysis

\begin{tabular}{|c|c|c|c|c|c|c|c|c|}
\hline & \multicolumn{4}{|c|}{ Implicit threshold } & \multicolumn{4}{|c|}{ Actual threshold } \\
\hline & \multicolumn{2}{|c|}{ Carbon footprint } & \multicolumn{2}{|c|}{ Kilocalories } & \multicolumn{2}{|c|}{ Carbon footprint } & \multicolumn{2}{|l|}{ Kilocalories } \\
\hline & Good & Bad & Healthy & Unhealthy & Good & Bad & Healthy & Unhealthy \\
\hline $\mathrm{C}$ & $221.85^{* *}$ & $482.95 * *$ & $327.50 * *$ & $475.78^{* *}$ & 16.17 & $1020.19 * *$ & $517.60 * *$ & $880.41 * *$ \\
\hline S.E. & 13.90 & 16.63 & 15.78 & 19.911 & 61.11 & 73.976 & 44.31 & 68.734 \\
\hline TP & $252.18^{* *}$ & $446.03 * *$ & $302.20 * *$ & $428.56^{* *}$ & $155.39 * *$ & $1021.15^{* *}$ & $516.90 * *$ & $895.14 * *$ \\
\hline S. E. & 12.35 & 16.77 & 13.63 & 17.754 & 64.99 & 76.2 & 47.04 & 75.685 \\
\hline $\mathbf{L}$ & $288.63^{* *}$ & $422.61 * *$ & $294.70 * *$ & $406.09^{* *}$ & $350.97 * *$ & $615.74 * *$ & $385.70^{* *}$ & $650.89^{* *}$ \\
\hline S. E. & 10.99 & 12.44 & 12.32 & 16.421 & 17.12 & 19.535 & 23.38 & 38.836 \\
\hline $\mathbf{L}+\mathbf{T P}$ & $273.37 * *$ & $395.94 * *$ & $318.80 * *$ & $398.84^{* *}$ & $354.35^{* *}$ & $625.94 * *$ & $449.30 * *$ & $627.25^{* *}$ \\
\hline S. E. & 10.75 & 12.79 & 13.55 & 15.456 & 20.39 & 23.369 & 31.07 & 40.005 \\
\hline Wald test & & & & & & & & \\
\hline $\mathbf{C}=\mathbf{T P}$ & 2.66 & 2.45 & 1.49 & 3.15 & 2.45 & 0.00 & 0.00 & 0.02 \\
\hline $\mathbf{C}=\mathbf{L}$ & $14.24 * *$ & $8.44^{* *}$ & 2.70 & $7.32 * *$ & $27.93 * *$ & $28.00^{* *}$ & $6.94 * *$ & $8.45^{* *}$ \\
\hline $\mathbf{C}=\mathbf{L}+\mathbf{T P}$ & $8.61 * *$ & $17.17 * *$ & 0.18 & $9.37 * *$ & $27.63 * *$ & $25.86^{* *}$ & 1.59 & $10.13^{* *}$ \\
\hline $\mathbf{T P}=\mathbf{L}+\mathbf{T P}$ & 1.68 & $5.63^{*}$ & 0.75 & 1.60 & $8.56^{* *}$ & $24.61^{* *}$ & 1.44 & $9.79^{* *}$ \\
\hline $\mathbf{L}=\mathbf{L}+\mathbf{T P}$ & 0.99 & 2.23 & 1.73 & 0.10 & 0.02 & 0.11 & 2.68 & 0.18 \\
\hline
\end{tabular}


Note: S.E. refers to standard errors estimated using the Delta method. Significance is as follows: ${ }^{*} \mathrm{p}<0.05 ; * * \mathrm{p}<0.01$. Acronyms are as follows: $\mathrm{B}=$ Baseline; $\mathrm{TP}=$ Time pressure; $\mathrm{L}=$ Labelling; $\mathrm{L}+\mathrm{TP}=$ joint Time pressure and Labelling. The thresholds for kilocalories exclude olive oil (see appendix 4 for the thresholds including olive oil). 


\section{APPENDIX 1: Full list of products}

Table A1: Full list of products (values per 100g)

\begin{tabular}{|c|c|c|c|c|}
\hline ID & Category & Product name & Carbon footprint & Keal \\
\hline 1 & Vegetables & Carrots Class 1 Pack $(1 \mathrm{~kg})$ & 83 & 42 \\
\hline 2 & & Market Value Carrots 1 Pack (1 kg) & 81 & 42 \\
\hline 3 & & Yorkshire Carrots Class 1 Pack (1 kg) & 79 & 42 \\
\hline 4 & & Cucumber Whole (360g) & 133 & 11 \\
\hline 5 & & Yorkshire Cucumber Whole (360g) & 163 & 11 \\
\hline 6 & & Closed Cup Mushrooms 250g & 480 & 16 \\
\hline 7 & & Dorset Closed Cup Mushrooms 250g & 560 & 16 \\
\hline 8 & & Value Closed Cup Mushrooms 250g & 470 & 16 \\
\hline 9 & Frozen peas & Frozen Petits Pois $1 \mathrm{~kg}$ & 125 & 59 \\
\hline 10 & & Frozen Organic Petit Pois $1 \mathrm{~kg}$ & 225 & 59 \\
\hline 11 & Baked beans & Baked Beans in Tomato Sauce 420g & 130 & 87 \\
\hline 12 & & Light Choice Baked Beans in tomato sauce $420 \mathrm{~g}$ & 130 & 70 \\
\hline 13 & & Value Baked Beans in Tomato Sauce 420g & 140 & 87 \\
\hline 14 & Olive oil & Extra Virgin Olive Oil $500 \mathrm{ml}$ & 467 & 900 \\
\hline 15 & & Organic Extra Virgin Olive Oil $500 \mathrm{ml}$ & 360 & 900 \\
\hline 16 & Pasta & Whole wheat Spaghetti 500g & 293 & 346 \\
\hline 17 & & Value Spaghetti (500g) & 320 & 351 \\
\hline 18 & & Spaghetti $500 \mathrm{~g}$ & 320 & 360 \\
\hline 19 & & Organic Spaghetti 500g & 320 & 350 \\
\hline 20 & & Organic Whole wheat spaghetti $500 \mathrm{~g}$ & 320 & 340 \\
\hline 21 & Prepared meals & Braised Beef \& Mash 450g & 810 & 101 \\
\hline 22 & & Light Choice Braised Beef and Mash 450g & 990 & 73 \\
\hline 23 & & Chilli con carne and rice $500 \mathrm{~g}$ & 1070 & 116 \\
\hline 24 & & Light Choices Chilli Con Carne \& Rice 500g & 900 & 100 \\
\hline 25 & & Cottage Pie $450 \mathrm{~g}$ & 1040 & 106 \\
\hline 26 & & Light Choices Cottage Pie 450g & 900 & 82 \\
\hline 27 & & Value Cottage Pie 450g & 750 & 73 \\
\hline 28 & Soya milk & Sweetened Soya milk (1 litre) & 80 & 42 \\
\hline 29 & & Unsweetened Soya milk (1 litre) & 70 & 34 \\
\hline 30 & & Value Unsweetened Soya milk (1 litre) & 70 & 34 \\
\hline 31 & & Organic Unsweetened Soya milk (1 litre) & 120 & 34 \\
\hline 32 & & Organic Sweetened Soya milk (1 litre) & 140 & 42 \\
\hline 33 & Milk & UHT value skimmed milk (1 litre) & 123 & 50 \\
\hline 34 & & UHT whole milk (1 litre) & 158 & 65 \\
\hline 35 & & UHT skimmed milk (1 litre) & 123 & 50 \\
\hline 36 & Eggs & Barn Medium eggs (6-pack) & 413 & 131 \\
\hline 37 & & Organic Medium eggs (6-pack) & 454 & 151 \\
\hline 38 & & Free-Range Medium eggs (6-pack) & 406 & 131 \\
\hline 39 & & Value Medium eggs (6-pack) & 451 & 131 \\
\hline 40 & Cola & Cola 21 bottle & 26 & 39 \\
\hline 41 & & Diet Cola 21 bottle & 22 & 0 \\
\hline 42 & Butter & English Unsalted Butter & 950 & 747 \\
\hline 43 & & English Salted Butter & 950 & 740 \\
\hline
\end{tabular}


Figure A1: Distribution of the number of values used in the metric assessment
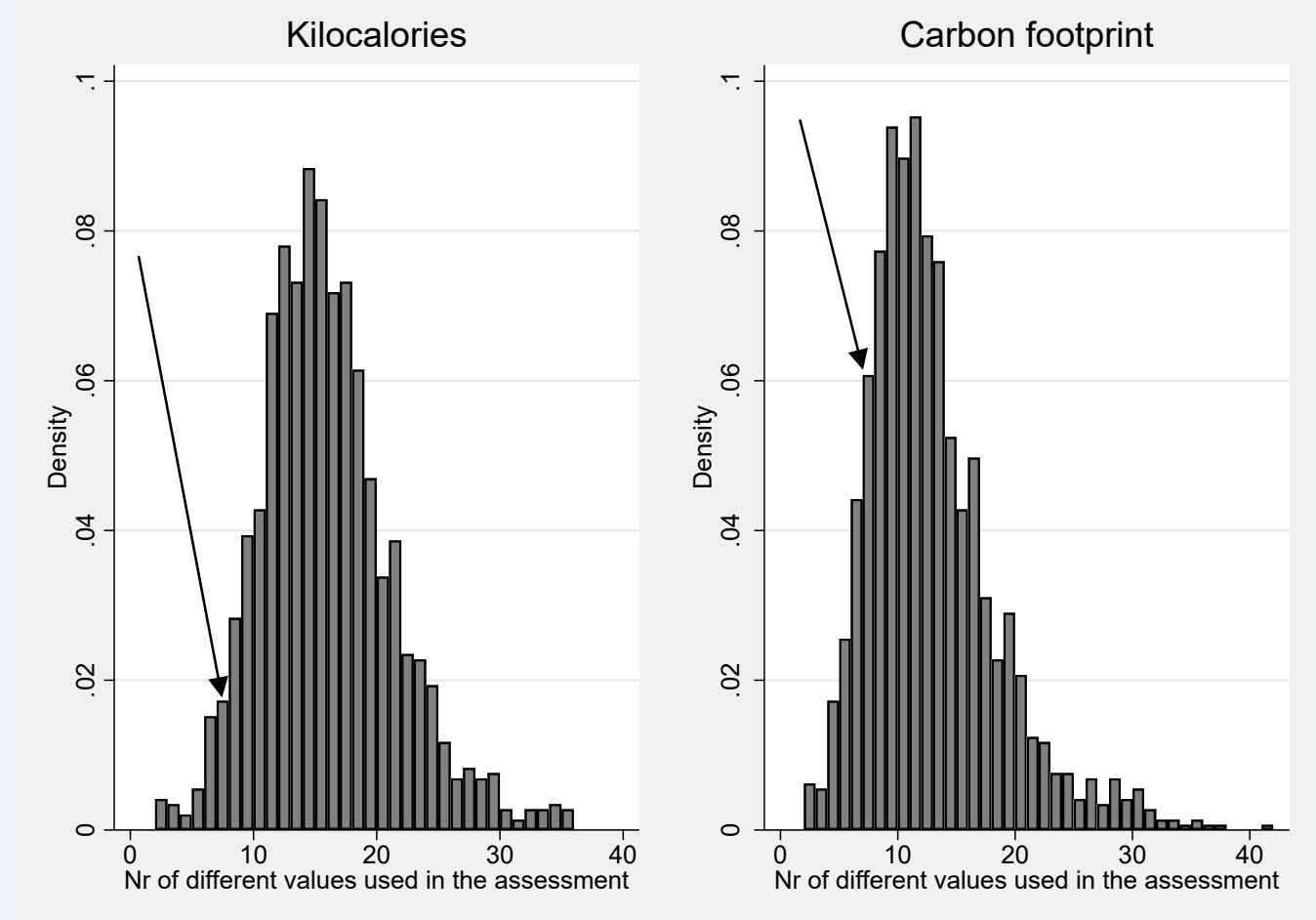

Note: graph does not include 1 (individuals who used the same value for all products). The arrows indicate the cut-off value of 7 different numbers, a criteria used to determine Insufficient-Effort-Response individuals.

Table A2: Time taken per evaluation, bottom five (the fastest) percentiles

\begin{tabular}{ll|ccccc}
\hline & \multicolumn{7}{|l}{ Percentile } & & & \\
\hline & Treatment & $\mathbf{1}$ & $\mathbf{2}$ & $\mathbf{3}$ & $\mathbf{4}$ & $\mathbf{5}$ \\
\hline CO2 & Control & 1.97 & 2.33 & 2.74 & $\mathbf{2 . 9 2}$ & 3.21 \\
& Time pressure & 1.51 & 2.25 & 2.67 & $\mathbf{2 . 9 0}$ & 3.17 \\
& Labelling & 2.21 & 2.63 & 2.90 & $\mathbf{3 . 2 0}$ & 3.47 \\
& Labelling + time pressure & 2.06 & 2.59 & 2.86 & $\mathbf{3 . 0 6}$ & 3.27 \\
\hline Kcal & Control & 1.92 & 2.75 & 3.09 & $\mathbf{3 . 3 9}$ & 3.63 \\
& Time pressure & 1.85 & 2.51 & 2.85 & $\mathbf{3 . 0 9}$ & 3.26 \\
& Labelling & 2.06 & 2.63 & 2.94 & $\mathbf{3 . 3 0}$ & 3.57 \\
& Labelling + time pressure & 2.43 & 2.89 & 3.32 & $\mathbf{3 . 5 2}$ & 3.77 \\
\hline
\end{tabular}

Note: cut-off points for each metric and treatment are identified in bold and italic. 
Table A3: Final sample characteristics

\begin{tabular}{|c|c|c|c|c|c|c|c|c|c|c|}
\hline \multirow{2}{*}{ Sample } & \multirow[b]{2}{*}{ Variable } & \multicolumn{2}{|l|}{$\mathbf{C}$} & \multicolumn{2}{|l|}{$\mathbf{T P}$} & \multirow{2}{*}{$\begin{array}{c}\text { L } \\
\text { Mean }\end{array}$} & \multicolumn{3}{|c|}{$\mathbf{L}+\mathbf{T P}$} & \multirow{2}{*}{$\begin{array}{c}\text { K-W test } \chi 2 \\
\quad \text { Mean }\end{array}$} \\
\hline & & Mean & S.D. & Mean & S.D. & & S.D. & Mean & S.D. & \\
\hline Carbon & Age & 48.56 & 17.70 & 48.14 & 17.60 & 48.04 & 17.33 & 49.02 & 17.52 & 0.71 \\
\hline \multirow[t]{10}{*}{ footprint } & Male & 0.45 & 0.50 & 0.46 & 0.50 & 0.48 & 0.50 & 0.47 & 0.50 & 0.81 \\
\hline & Environmental attitudes & 4.04 & 0.90 & 4.16 & 0.80 & 4.14 & 0.87 & 4.19 & 0.82 & 3.84 \\
\hline & Health attitudes & 3.83 & 1.00 & 3.82 & 1.03 & 3.91 & 0.95 & 4.01 & 1.00 & 7.48 \\
\hline & Environmental identity & 3.78 & 1.00 & 3.80 & 0.97 & 3.72 & 0.99 & 3.82 & 0.98 & 1.59 \\
\hline & Health identity & 3.42 & 1.03 & 3.34 & 1.08 & 3.41 & 1.01 & 3.44 & 1.05 & 1.54 \\
\hline & Footprint knowledge & 0.89 & 0.71 & 0.84 & 0.70 & 0.86 & 0.68 & 0.81 & 0.71 & 2.21 \\
\hline & Kilocalories knowledge & 0.75 & 0.72 & 0.75 & 0.70 & 0.83 & 0.71 & 0.85 & 0.73 & 4.74 \\
\hline & Frequency - environment & 0.90 & 1.08 & 0.92 & 1.10 & 0.92 & 1.09 & 0.89 & 1.00 & 0.10 \\
\hline & Survey time (min) & 34.93 & 58.34 & 19.90 & 10.22 & 47.00 & 141.18 & 31.48 & 81.19 & 148.33 \\
\hline & Observations & 296 & & 318 & & 325 & & 279 & & \\
\hline \multirow[t]{11}{*}{ Kilocalories } & Age & 48.33 & 17.52 & 48.19 & 17.37 & 48.56 & 17.06 & 48.91 & 17.14 & 0.37 \\
\hline & Male & 0.42 & 0.49 & 0.45 & 0.50 & 0.47 & 0.50 & 0.48 & 0.50 & 3.13 \\
\hline & Environmental attitudes & 4.07 & 0.88 & 4.15 & 0.80 & 4.11 & 0.90 & 4.16 & 0.84 & 1.96 \\
\hline & Health attitudes & 3.83 & 0.99 & 3.78 & 1.03 & 3.89 & 1.00 & 3.98 & 1.02 & $8.96^{* *}$ \\
\hline & Environmental identity & 3.74 & 1.01 & 3.77 & 0.98 & 3.72 & 1.02 & 3.76 & 1.03 & 0.63 \\
\hline & Health identity & 3.35 & 1.05 & 3.30 & 1.07 & 3.39 & 1.05 & 3.40 & 1.06 & 2.51 \\
\hline & Footprint knowledge & 0.85 & 0.72 & 0.81 & 0.68 & 0.84 & 0.69 & 0.80 & 0.72 & 1.21 \\
\hline & Kilocalories knowledge & 0.76 & 0.72 & 0.76 & 0.71 & 0.83 & 0.71 & 0.81 & 0.72 & 2.96 \\
\hline & Frequency - health & 1.35 & 1.22 & 1.37 & 1.34 & 1.38 & 1.25 & 1.35 & 1.24 & 0.97 \\
\hline & Survey time (min) & 34.30 & 56.54 & 24.53 & 99.01 & 45.19 & 134.24 & 30.87 & 78.75 & 158.59 \\
\hline & Observations & 350 & & 371 & & 361 & & 321 & & \\
\hline
\end{tabular}




\section{APPENDIX 3: Modelling information}

\section{Section 3.6.3 - Additional methodological details}

The relationship between estimates and actual values can be further assessed using a multivariate regression that regresses the estimate $y$ of the true value $y^{*}$ as (Attari et al. 2010)

$$
\log _{10}\left(y_{i j}\right)=\gamma_{0 i}+\gamma_{1 i} \log _{10}\left(y_{j}^{*}\right)+\gamma_{2 i}\left[\log _{10}\left(y_{i j}^{*}\right)\right]^{2}+\varepsilon_{i j}
$$

Equation (A1) allows for all coefficients (and biases) to be subjective, varying with two parameters: the experimental group $I_{i}$ and personal characteristics $z i$, so that $\gamma_{0 i}=\bar{\gamma}_{0}+\pi_{0} I_{i}+\theta_{0} z_{i}+e_{0 i}, \gamma_{1 i}=$ $\bar{\gamma}_{1}+\pi_{1} I_{i}+\theta_{1} z_{i}+e_{1 i}$ and $\gamma_{2 i}=\bar{\gamma}_{1}+\pi_{2} I_{i}+\theta_{2} z_{i}+e_{2 i}$. By centering $\log _{10}\left(y_{i j}\right), \log _{10}\left(y_{j}^{*}\right)$, and $\left[\log _{10}\left(y_{i j}^{*}\right)\right]^{2}$ by the mean of $\log _{10}\left(y_{i j}^{*}\right)$, the coefficients directly quantify deviations from the mean of the actual value: at the mean of $\log _{10}\left(y_{i j}^{*}\right)$, both transformed dependent variables are zero, so that $\gamma_{0 i}$ indicates a true deviation from the actual value.

\section{Section 3.7 - Additional methodological details}

The binary probit approach defines a threshold as the point along the perceptual spectrum where $50 \%$ of participants switch from a category to the other (Bi and Ennis 1998; Lawless and Heymann 2010; Logvinenko et al. 2012). In the categorisation task, participant $i$ classifies a product $j$ as positive ("healthy", "good for the environment") if the expected impact (in kilocalories or GHGs) $y_{i j}$ is below a lower threshold $\bar{y}^{L}$ the respondent has in mind; or negative ("unhealthy", "bad for the environment") if the expected impact $y_{i j}$ is above a higher threshold $\bar{y}^{H}$. The categorisation then assigns a dummy $G_{i j}=1$ if the product is classified as "Good for the environment" (in the carbon footprint equation) or "Healthy" (in the kilocalories equation), 0 otherwise; and a dummy $B_{i j}=1$ if a product is classified as "Bad for the environment" (in the carbon footprint equation) or "Unhealthy" (in the kilocalories equation), 0 otherwise. The resulting probit specifications correspond to

$$
P\left(G_{i j}=1\right)=P\left(y_{i j}<\bar{y}^{L}\right)=\Phi\left(\beta_{0 i}^{L}+\beta_{1 i}^{L} y_{i j}+e_{i j}^{L}\right)
$$




$$
P\left(B_{i j}=1\right)=P\left(y_{i j}>\bar{y}^{H}\right)=\Phi\left(\beta_{0 i}^{H}+\beta_{1 i}^{H} y_{i j}+e_{i j}^{H}\right)
$$

where $\Phi$ is the standard normal cdf.

To understand how thresholds can be obtained from a probit model, re-write condition (A3) as

$$
\beta_{0 i}^{H}+\beta_{1 i}^{H} y_{i j}+e_{i j}^{H}>0
$$

if $B_{i j}=1, B_{i j}=0$ otherwise. The higher threshold can be estimated by solving (A4) for $y_{i j}$ as

$$
y_{i j}>-\frac{\beta_{0 i}^{H}}{\beta_{1 i}^{H}}-e_{i j}^{H}=-\bar{y}^{H}-e_{i j}^{H}
$$

The same approach obtains the lower threshold $\bar{y}^{L}$ from condition (A2), starting from the inequality $\beta_{0 i}^{L}+\beta_{1 i}^{L} y_{i j}+e_{i j}^{L}<0$. The final thresholds are (Knoblauch and Maloney 2012, page 154)

$$
\begin{array}{ll}
\bar{y}^{L}=-\frac{\bar{\beta}_{0}^{L}}{\bar{\beta}_{1}^{L}} & \text { (lower threshold) } \\
\bar{y}^{H}=-\frac{\bar{\beta}_{0}^{H}}{\bar{\beta}_{1}^{H}} & \text { (higher threshold) }
\end{array}
$$

In the article, we estimate two random intercept multi-level probit regressions. We allow for subjective thresholds by allowing the intercept to vary with the experimental group $I$, and unobservable individual characteristics $\omega_{i}$, so that $\beta_{0 i}^{H}=\bar{\beta}_{0}^{H}+\delta_{0}^{H} I_{i}+\omega_{i}^{H}$ and $\beta_{0 i}^{L}=\bar{\beta}_{0}^{L}+\delta_{0}^{L} I_{i}+$ $\omega_{i}^{L}$. We also allow for treatment-specific slopes $\beta_{1 i}^{L}=\bar{\beta}_{1}^{L}+\delta_{1}^{L} I_{i}$ and $\beta_{1 i}^{H}=\bar{\beta}_{1}^{H}+\delta_{1}^{H} I_{i}$. The addition of a random slope is possible at a significant computational burden, because the likelihood function has no closed form and requires approximation. A linear mixed model on both regressions indicates that the random slope has a variance close to zero, suggesting its inclusion is unnecessary. The estimated equations are

$$
\begin{aligned}
& G_{i j}=\bar{\beta}_{0}^{L}+\delta_{0}^{L} I_{i}+\bar{\beta}_{1}^{L} y_{i j}+\delta_{1}^{L} I_{i} y_{i j}+\omega_{i}^{L}+e_{i j}^{L} \\
& B_{i j}=\bar{\beta}_{0}^{H}+\delta_{0}^{H} I_{i}+\bar{\beta}_{1}^{H} y_{i j}+\delta_{1}^{H} I_{i} y_{i j}+\omega_{i}^{H}+e_{i j}^{H}
\end{aligned}
$$

In the presence of a manipulation, the lower thresholds then corresponds to

$$
\bar{y}^{L}=-\left(\frac{\bar{\beta}_{0}^{L}+\delta_{0}}{\bar{\beta}_{1}^{L}+\delta_{1}}\right)
$$


while the higher thresholds is

$$
\bar{y}^{H}=-\left(\frac{\bar{\beta}_{0}^{H}+\delta_{0}}{\bar{\beta}_{1}^{H}+\delta_{1}}\right)
$$

The estimation of actual thresholds, the variable $y_{i j}$ in equations (A6) and (A7) is replaced by $y_{i j}^{*}$. 


\section{APPENDIX 4: Additional information on the categorisation task}

Figure A2: Stimulus-response curve, by metric and treatment

a) Carbon footprint
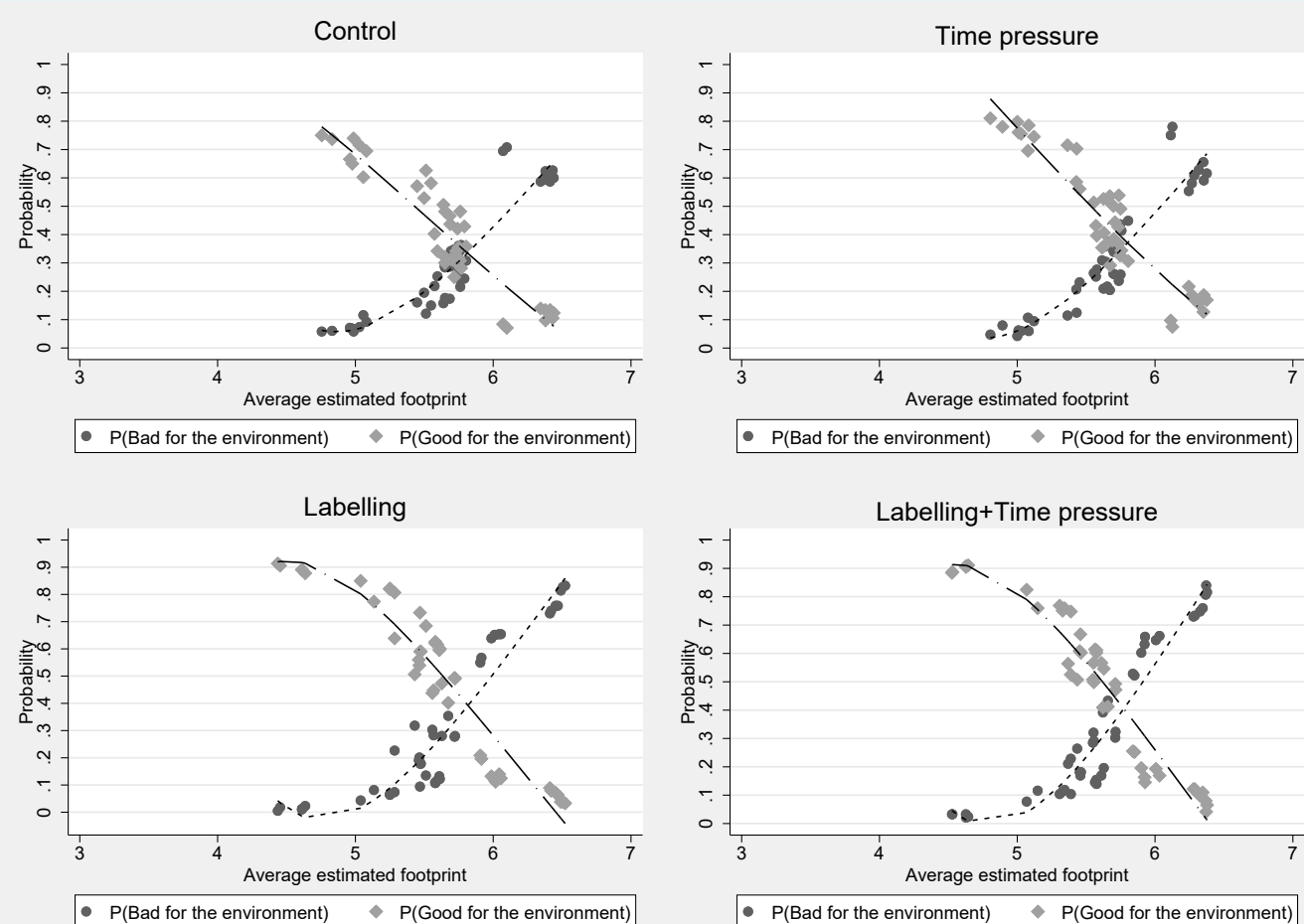

b) Kilocalories
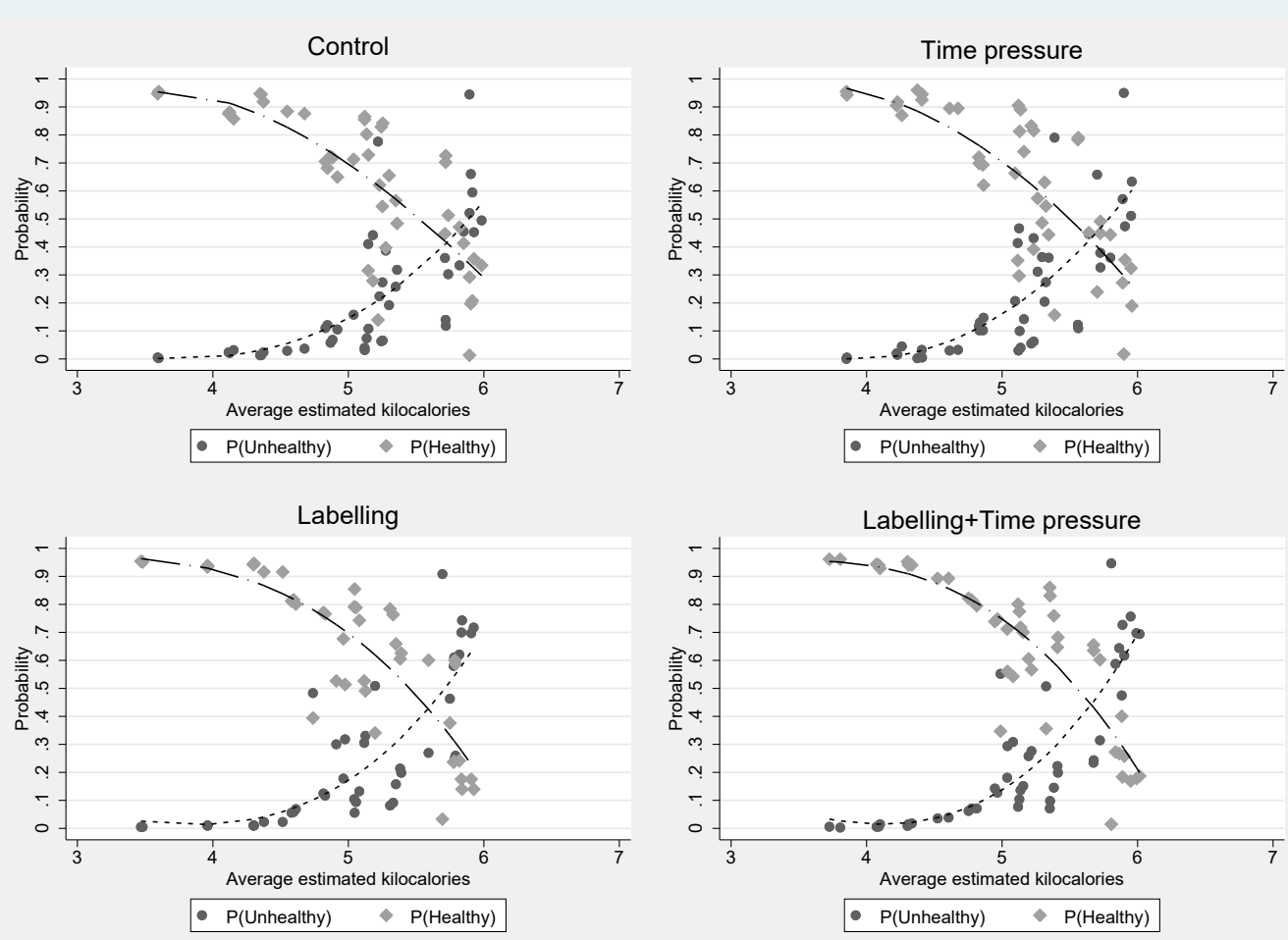

Note: fitted variables refer to fractional polynomial prediction plots. The $\mathrm{x}$ axis is in natural logarithmic form. 
Table A4: Percentage of positive and negative classifications, by food category

\begin{tabular}{|c|c|c|c|c|c|}
\hline Category & Classification & Control & Time pressure & Labelling & $\begin{array}{c}\text { Labelling + } \\
\text { Time pressure }\end{array}$ \\
\hline \multirow[t]{4}{*}{ Baked beans } & $\%$ Good for the environment & $30.26 \%$ & $32.59 \%$ & $81.59 \%$ & $75.96 \%$ \\
\hline & $\%$ Bad for the environment & $34.74 \%$ & $43.31 \%$ & $6.79 \%$ & $11.18 \%$ \\
\hline & \% Healthy & $62.63 \%$ & $57.44 \%$ & $56.49 \%$ & $62.81 \%$ \\
\hline & $\%$ Unhealthy & $21.84 \%$ & $29.43 \%$ & $27.14 \%$ & $23.84 \%$ \\
\hline \multirow[t]{4}{*}{ Butter } & $\%$ Good for the environment & $38.95 \%$ & $46.38 \%$ & $13.23 \%$ & $18.10 \%$ \\
\hline & $\%$ Bad for the environment & $27.76 \%$ & $29.93 \%$ & $65.39 \%$ & $65.43 \%$ \\
\hline & \% Healthy & $30.53 \%$ & $34.54 \%$ & $20.87 \%$ & $22.55 \%$ \\
\hline & \% Unhealthy & $55.79 \%$ & $55.24 \%$ & $66.03 \%$ & $68.55 \%$ \\
\hline \multirow[t]{4}{*}{ Cola } & \% Good for the environment & $7.76 \%$ & $8.60 \%$ & $57.25 \%$ & $48.96 \%$ \\
\hline & $\%$ Bad for the environment & $70.13 \%$ & $76.56 \%$ & $27.23 \%$ & $37.69 \%$ \\
\hline & \% Healthy & $7.63 \%$ & $8.73 \%$ & $21.37 \%$ & $18.10 \%$ \\
\hline & $\%$ Unhealthy & $86.05 \%$ & $87.03 \%$ & $69.59 \%$ & $74.93 \%$ \\
\hline \multirow[t]{4}{*}{ Eggs } & \% Good for the environment & $50.00 \%$ & $58.48 \%$ & $57.32 \%$ & $58.31 \%$ \\
\hline & $\%$ Bad for the environment & $22.96 \%$ & $21.51 \%$ & $21.56 \%$ & $23.74 \%$ \\
\hline & \% Healthy & $81.32 \%$ & $83.73 \%$ & $79.45 \%$ & $74.85 \%$ \\
\hline & $\%$ Unhealthy & $6.32 \%$ & $7.79 \%$ & $9.67 \%$ & $11.72 \%$ \\
\hline \multirow[t]{4}{*}{ Frozen peas } & \% Good for the environment & $55.00 \%$ & $57.36 \%$ & $81.17 \%$ & $79.23 \%$ \\
\hline & $\%$ Bad for the environment & $17.76 \%$ & $21.95 \%$ & $6.23 \%$ & $9.64 \%$ \\
\hline & \% Healthy & $88.03 \%$ & $89.53 \%$ & $91.60 \%$ & $89.32 \%$ \\
\hline & $\%$ Unhealthy & $3.29 \%$ & $3.12 \%$ & $2.29 \%$ & $3.71 \%$ \\
\hline \multirow[t]{4}{*}{ Milk } & \% Good for the environment & $30.96 \%$ & $38.24 \%$ & $54.28 \%$ & $56.97 \%$ \\
\hline & $\%$ Bad for the environment & $34.39 \%$ & $34.41 \%$ & $21.29 \%$ & $21.76 \%$ \\
\hline & \% Healthy & $59.47 \%$ & $60.43 \%$ & $62.60 \%$ & $61.42 \%$ \\
\hline & \% Unhealthy & $20.61 \%$ & $22.61 \%$ & $25.02 \%$ & $25.91 \%$ \\
\hline \multirow[t]{4}{*}{ Olive oil } & \% Good for the environment & $45.53 \%$ & $51.50 \%$ & $49.24 \%$ & $48.22 \%$ \\
\hline & $\%$ Bad for the environment & $23.03 \%$ & $24.81 \%$ & $27.86 \%$ & $31.31 \%$ \\
\hline & \% Healthy & $71.45 \%$ & $78.68 \%$ & $59.41 \%$ & $64.54 \%$ \\
\hline & $\%$ Unhealthy & $12.89 \%$ & $11.60 \%$ & $25.57 \%$ & $23.89 \%$ \\
\hline \multirow[t]{4}{*}{ Pasta } & $\%$ Good for the environment & $42.16 \%$ & $47.98 \%$ & $61.17 \%$ & $58.81 \%$ \\
\hline & $\%$ Bad for the environment & $20.95 \%$ & $24.49 \%$ & $11.76 \%$ & $16.08 \%$ \\
\hline & \% Healthy & $67.53 \%$ & $65.39 \%$ & $68.75 \%$ & $75.61 \%$ \\
\hline & \% Unhealthy & $17.95 \%$ & $19.15 \%$ & $14.86 \%$ & $14.72 \%$ \\
\hline \multirow[t]{4}{*}{ Ready meals } & \% Good for the environment & $12.29 \%$ & $17.39 \%$ & $5.89 \%$ & $9.11 \%$ \\
\hline & $\%$ Bad for the environment & $60.23 \%$ & $60.49 \%$ & $78.01 \%$ & $77.62 \%$ \\
\hline & \% Healthy & $37.48 \%$ & $36.05 \%$ & $27.26 \%$ & $29.55 \%$ \\
\hline & \% Unhealthy & $43.72 \%$ & $46.53 \%$ & $58.31 \%$ & $59.18 \%$ \\
\hline \multirow[t]{4}{*}{ Soya milk } & $\%$ Good for the environment & $32.32 \%$ & $36.81 \%$ & $15.37 \%$ & $20.24 \%$ \\
\hline & $\%$ Bad for the environment & $27.84 \%$ & $30.37 \%$ & $61.17 \%$ & $58.87 \%$ \\
\hline & \% Healthy & $53.74 \%$ & $53.22 \%$ & $69.41 \%$ & $70.74 \%$ \\
\hline & \% Unhealthy & $21.68 \%$ & $24.64 \%$ & $16.03 \%$ & $16.14 \%$ \\
\hline \multirow[t]{4}{*}{ Vegetables } & \% Good for the environment & $69.44 \%$ & $76.65 \%$ & $75.19 \%$ & $76.15 \%$ \\
\hline & $\%$ Bad for the environment & $7.47 \%$ & $6.92 \%$ & $9.54 \%$ & $10.57 \%$ \\
\hline & $\%$ Healthy & $91.61 \%$ & $92.77 \%$ & $94.34 \%$ & $94.62 \%$ \\
\hline & \% Unhealthy & $1.68 \%$ & $1.56 \%$ & $0.89 \%$ & $0.96 \%$ \\
\hline
\end{tabular}


Figure A3: Estimated kilocalories thresholds, all products

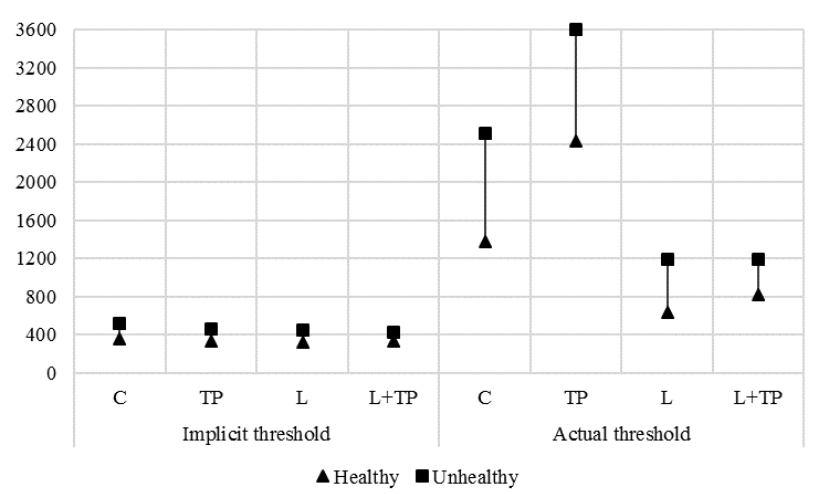

Table A5: Estimated thresholds - all products (including olive oil)

\begin{tabular}{|c|c|c|c|c|}
\hline & \multicolumn{2}{|c|}{ Implicit threshold } & \multicolumn{2}{|c|}{ Actual threshold } \\
\hline & Healthy & Unhealthy & Healthy & Unhealthy \\
\hline $\mathbf{C}$ & $357.94 * *$ & $520.39 * *$ & $1372.49 * *$ & $2514.39 * *$ \\
\hline S.E. & 17.68 & 21.93 & 257.82 & 474.52 \\
\hline TP & $327.73 * *$ & $464.20 * *$ & $2426.28 * *$ & $3596.67 * *$ \\
\hline S. E. & 15.18 & 19.67 & 796.03 & 976.90 \\
\hline $\mathbf{L}$ & $319.40 * *$ & $451.02 * *$ & $637.42 * *$ & $1185.15^{* *}$ \\
\hline S. E. & 13.34 & 18.26 & 49.33 & 106.96 \\
\hline $\mathbf{L}+\mathbf{T P}$ & $335.70 * *$ & $421.49 * *$ & $813.62 * *$ & $1194.75^{* *}$ \\
\hline S. E. & 14.46 & 16.58 & 79.91 & 117.71 \\
\hline \multicolumn{5}{|l|}{ Wald test } \\
\hline $\mathbf{C}=\mathbf{T P}$ & 1.70 & 3.66 & 1.59 & 0.99 \\
\hline $\mathbf{C}=\mathbf{L}$ & 3.05 & $5.93^{*}$ & $7.84 * *$ & $7.47 * *$ \\
\hline $\mathbf{C}=\mathbf{L}+\mathbf{T P}$ & 0.96 & $13.02 * *$ & $4.29 *$ & $7.29 * *$ \\
\hline $\mathbf{T P}=\mathbf{L}+\mathbf{T P}$ & 0.15 & 2.77 & $4.06^{*}$ & $5.96^{*}$ \\
\hline $\mathbf{L}=\mathbf{L}+\mathbf{T P}$ & 0.69 & 1.44 & 3.52 & 0.00 \\
\hline
\end{tabular}

Note: S.E. refers to standard errors estimated using the Delta method. Significance is as follows: $* \mathrm{p}<0.05 ; * * \mathrm{p}<0.01$. Acronyms are as follows: $\mathrm{B}=$ Baseline; $\mathrm{TP}=$ Time pressure; $\mathrm{L}=$ Labelling; $\mathrm{L}+\mathrm{TP}=$ joint Time pressure and Labelling. 
Table A6: Multi-level probit regression estimates of the categorisation task






\section{ONLINE APPENDIX 1: Full questionnaire}

Explanation of Kilocalories [randomise order of information, presented in different pages]

A kilocalorie is a measure of how much energy you get from a certain quantity of food. Calories from carbohydrates, proteins, and fats provide energy that allows the human body to perform all its functions, such as breathe, engage in physical activity (walking, running), and support growth in infants and children. Other nutrients (e.g. vitamins and minerals) and water are essential parts of a healthy diet, but do not provide energy. Eating too many calories leads to weight gain and obesity, which are associated with several diseases (e.g., heart disease, cancer). Nutrition labels generally present information on carbohydrates (which include sugars), fat, and salt, and use traffic-light colours to allow consumers to identify harmful levels of each, as shown below.

\begin{tabular}{l|l|l|l} 
Nutrient content (100 g) & $\begin{array}{l}\text { Carbohydrates } \\
\text { (incl. sugars) }\end{array}$ & Fats & Salt \\
\hline $\begin{array}{l}\text { Orange Juice Not From Concentrate } \\
\text { (Ambient) (1 I) }\end{array}$ & $17.0 \mathrm{~g}$ & $0 \mathrm{~g}$ & $0 \mathrm{~g}$ \\
\hline
\end{tabular}

The recommended daily calories intake is 1,800 kilocalories for women and 2,000 kilocalories for men.

\section{Explanation of Carbon Footprint}

The carbon footprint of a food is the amount of greenhouse gases emitted to produce and consume the food. It is expressed in tonnes of carbon dioxide equivalents, or "tonnes of $\mathrm{CO}_{2} \mathrm{e}$ " for short. The carbon footprint measures the impact of the consumption of a product on global warming by summing all emissions of gases from the production of raw materials, industrial processing, transport, storage, cooking, and consumption. For instance, eating an egg emits $\mathrm{CO}_{2}$ to: make and deliver feed for the hens; heat the shed to maintain the hens healthy; transport the eggs by van; store eggs in chilled rooms; produce the packaging in which eggs are sold; and use energy for cooking it. A possible carbon footprint label can present individuals with information of the level of emissions the product requires at each stage of the food chain, as shown below.

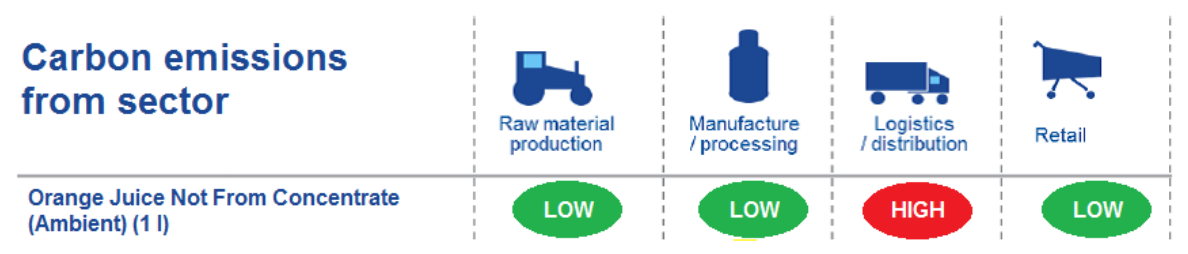

There are currently no agreed guidelines on the optimal daily amount of carbon footprint. 
STEP 1: Rate the Environmental Impact of Food (randomise order of step 1 and 2; randomise the order of each box)

According to your knowledge and opinion, how damaging would you say each of these foods are towards the environment? Remember that a higher carbon footprint indicates a higher damage.

Note that all products are currently sold in a large UK supermarket chain using their own brand.

The carbon footprint of this sample is below $1040 \mathrm{gCO} 2 \mathrm{e}$ per $100 \mathrm{~g}$ of product. Some examples:

(the table should be visible at all time during the task)

NOTE: this table must be visible throughout the task

\begin{tabular}{lc}
\hline Product name & CO $_{2}$ footprint (100g) \\
\hline Red Kidney Beans 500g & 300 \\
Cannellini Beans 500g & 300 \\
Wafer Thin Roast Chicken 205g & 720 \\
Plum Peeled Tomatoes (tin) 400g & 120 \\
Beef Lasagne 400g & 1000 \\
Curly Fries 400g & 260 \\
Wafer Thin Roast Chicken 450g & 680 \\
Corn Flakes 750g pack & 317 \\
Fresh Single Cream 150 ml & 336 \\
Still Water 2 litres bottle & 20 \\
\hline
\end{tabular}

\begin{tabular}{lcccc}
\hline Product name & $\begin{array}{c}\text { Good for the } \\
\text { environment }\end{array}$ & $\begin{array}{l}\text { Bad for the Unsure } \\
\text { environment }\end{array}$ & CO2 footprint (100g) \\
\hline Carrots Class 1 Pack (1 kg) & $\circ$ & $\circ$ & 0 & - \\
Market Value Carrots 1 Pack (1 kg) & $\circ$ & $\circ$ & 0 & - \\
Yorkshire Carrots Class 1 Pack (1 kg) & $\circ$ & $\circ$ & $\circ$ & - \\
\hline
\end{tabular}

\begin{tabular}{lcccc}
\hline Product name & $\begin{array}{c}\text { Good for the } \\
\text { environment }\end{array}$ & $\begin{array}{l}\text { Bad for the Unsure } \\
\text { environment }\end{array}$ & CO2 footprint (100g) \\
\hline Cucumber Whole (360g) & $\circ$ & 0 & 0 & - \\
Yorkshire Cucumber Whole (360g) & $\circ$ & $\circ$ & $\circ$ & - \\
& $\circ$ & $\circ$ & $\circ$ \\
\hline
\end{tabular}




\begin{tabular}{lccccc}
\hline Product name & $\begin{array}{l}\text { Good for the } \\
\text { environment }\end{array}$ & $\begin{array}{l}\text { Bad for the } \\
\text { environment }\end{array}$ & $\begin{array}{l}\text { Unsur } \\
\text { e }\end{array}$ & Cootprint (100g) \\
\hline Closed Cup Mushrooms 250g & 0 & 0 & & - \\
Dorset Closed Cup Mushrooms 250g & $\circ$ & 0 & \\
Value Closed Cup Mushrooms 250g & $\circ$ & 0 & - \\
\hline
\end{tabular}

\begin{tabular}{|c|c|c|c|c|}
\hline Product name & $\begin{array}{l}\text { Good for the } \\
\text { environment }\end{array}$ & $\begin{array}{l}\text { Bad for the } \\
\text { environment }\end{array}$ & Unsure & $\mathrm{CO}_{2}$ footprint $(100 \mathrm{~g})$ \\
\hline Tesco Frozen Petits Pois $1 \mathrm{~kg}$ & o & o & o & \\
\hline Tesco Frozen Organic Petit Pois 500g & ○ & ○ & ० & \\
\hline
\end{tabular}

\begin{tabular}{|c|c|c|c|c|}
\hline Product name & $\begin{array}{l}\text { Good for the } \\
\text { environment }\end{array}$ & $\begin{array}{l}\text { Bad for the } \\
\text { environment }\end{array}$ & Unsure & $\mathrm{CO}_{2}$ footprint $(100 \mathrm{~g})$ \\
\hline Baked Beans in Tomato Sauce 420g & o & o & o & \\
\hline Light Choice Baked Beans in tomato sauce $420 \mathrm{~g}$ & ○ & o & ○ & \\
\hline Value Baked Beans in Tomato Sauce $420 \mathrm{~g}$ & ○ & ○ & ○ & \\
\hline
\end{tabular}

\begin{tabular}{|c|c|c|c|c|}
\hline Product name & $\begin{array}{l}\text { Good for the } \\
\text { environment }\end{array}$ & $\begin{array}{l}\text { Bad for the } \\
\text { environment }\end{array}$ & Unsure & $\mathrm{CO}_{2}$ footprint $(100 \mathrm{~g})$ \\
\hline Extra Virgin Olive Oil $500 \mathrm{ml}$ & 0 & 0 & o & \\
\hline Organic Extra Virgin Olive Oil $500 \mathrm{ml}$ & ○ & ० & ○ & \\
\hline
\end{tabular}

\begin{tabular}{|c|c|c|c|c|}
\hline Product name & $\begin{array}{l}\text { Good for the } \\
\text { environment }\end{array}$ & $\begin{array}{l}\text { Bad for the } \\
\text { environment }\end{array}$ & Unsure & $\mathrm{CO}_{2}$ footprint (100g) \\
\hline Wholewheat Spaghetti 500g & 0 & 0 & 0 & \\
\hline Value Spaghetti (500g) & ० & ○ & ○ & \\
\hline Spaghetti 500g & o & ० & ० & \\
\hline Organic Spaghetti 500g & ० & ० & ○ & \\
\hline Organic Wholewheat spaghetti 500g & o & o & ○ & \\
\hline
\end{tabular}

\begin{tabular}{|c|c|c|c|c|}
\hline Product name & $\begin{array}{l}\text { Good for the } \\
\text { environment }\end{array}$ & $\begin{array}{l}\text { Bad for the } \\
\text { environment }\end{array}$ & Unsure & $\mathrm{CO}_{2}$ footprint $(100 \mathrm{~g})$ \\
\hline Braised Beef \& Mash 450g & o & o & o & \\
\hline Light Choice Braised Beef and Mash 450g & ○ & ○ & ○ & \\
\hline Chilli con carne and rice $500 \mathrm{~g}$ & $\circ$ & ○ & ○ & \\
\hline Light Choices Chilli Con Carne \& Rice 500g & ○ & ○ & ○ & \\
\hline
\end{tabular}




\begin{tabular}{|c|c|c|c|c|}
\hline Product name & $\begin{array}{l}\text { Good for the } \\
\text { environment }\end{array}$ & $\begin{array}{l}\text { Bad for the } \\
\text { environment }\end{array}$ & Unsure & $\mathrm{CO}_{2}$ footprint $(100 \mathrm{~g})$ \\
\hline Sweetened Soya milk (1 litre) & 0 & o & o & \\
\hline Unsweetened Soya milk (1 litre) & ○ & ○ & ○ & \\
\hline Value Unweetened Soya milk (1 litre) & $\circ$ & ○ & ○ & \\
\hline Organic Unsweetened Soya milk (1 litre) & $\circ$ & ○ & ○ & \\
\hline Organic Sweetened Soya milk (1 litre) & o & o & ० & \\
\hline
\end{tabular}

\begin{tabular}{lccccc}
\hline Product name & $\begin{array}{c}\text { Good for the } \\
\text { environment }\end{array}$ & $\begin{array}{l}\text { Bad for the Unsure } \\
\text { environment }\end{array}$ & CO2 footprint (100g) \\
\hline UHT value skimmed milk (1 litre) & 0 & 0 & 0 & - \\
UHT whole milk (1 litre) & 0 & 0 & 0 & - \\
UHT skimmed milk (1 litre) & $\circ$ & $\circ$ & 0 & - \\
\hline
\end{tabular}

\begin{tabular}{lcccc}
\hline Product name & $\begin{array}{c}\text { Good for the Bad for the Unsure } \\
\text { environment }\end{array}$ & $\begin{array}{l}\text { CO }_{2} \text { footprint (100g) } \\
\text { environment }\end{array}$ \\
\hline Barn Medium eggs (6-pack) & 0 & $\circ$ & 0 & - \\
Organic Medium eggs (6-pack) & $\circ$ & $\circ$ & $\circ$ & - \\
Free-Range Medium eggs (6-pack) & $\circ$ & $\circ$ & 0 & - \\
Value Medium eggs (6-pack) & $\circ$ & $\circ$ & $\circ$ & - \\
\hline
\end{tabular}

\begin{tabular}{|c|c|c|c|c|}
\hline Product name & $\begin{array}{l}\text { Good for the } \\
\text { environment }\end{array}$ & $\begin{array}{l}\text { Bad for the } \\
\text { environment }\end{array}$ & Unsure & $\mathrm{CO}_{2}$ footprint (100g) \\
\hline Cola 2 I bottle & 0 & 0 & 0 & \\
\hline Diet Cola 2 I bottle & o & o & o & \\
\hline
\end{tabular}

\begin{tabular}{|c|c|c|c|c|}
\hline Product name & $\begin{array}{l}\text { Good for the } \\
\text { environment }\end{array}$ & $\begin{array}{l}\text { Bad for the } \\
\text { environment }\end{array}$ & Unsure & $\mathrm{CO}_{2}$ footprint $(100 \mathrm{~g})$ \\
\hline English Unsalted Butter & o & 0 & o & \\
\hline English Salted Butter & o & o & o & \\
\hline
\end{tabular}

\begin{tabular}{|c|c|c|c|c|}
\hline Product name & $\begin{array}{l}\text { Good for the } \\
\text { environment }\end{array}$ & $\begin{array}{l}\text { Bad for the } \\
\text { environment }\end{array}$ & Unsure & $\mathrm{CO}_{2}$ footprint $(100 \mathrm{~g})$ \\
\hline Cottage Pie 450g & o & 0 & o & \\
\hline Light Choices Cottage Pie 450g & ○ & ○ & ० & \\
\hline Value Cottage Pie 450g & o & o & o & \\
\hline
\end{tabular}


STEP 2: Rate the Healthiness of foods (randomise order of step 1 and 2)

According to your knowledge and opinion, how damaging would you say each of these foods are towards health? Remember that more calories indicate a higher damage to health.

Note that all products are currently sold in a large UK supermarket chain using their own brand.

The calories content of this sample is below $\mathbf{1 0 0 0}$ kcal per $100 \mathrm{~g}$ of product. Some examples:

(the table should be visible at all time during the task)

NOTE: this table must be visible throughout the task

\begin{tabular}{lc}
\hline Product name & kcal (100g) \\
\hline Red Kidney Beans 500g & 108 \\
Cannellini Beans 500g & 98 \\
Wafer Thin Roast Chicken 205g & 101 \\
Plum Peeled Tomatoes (tin) 400g & 25 \\
Beef Lasagne al forno 400g & 183 \\
Curly Fries 400g & 243 \\
Wafer Thin Roast Chicken 450g & 101 \\
Corn Flakes 750g pack & 384 \\
Fresh Single Cream 150 ml & 194 \\
Still Water 2 litres bottle & 0 \\
\hline
\end{tabular}

\begin{tabular}{lcccl}
\hline Product name & Healthy & Unhealthy & Unsure & Calories content (100g) \\
\hline Carrots Class 1 Pack (1 kg) & $\circ$ & $\circ$ & $\circ$ & \\
Market Value Carrots 1 Pack (1 kg) & $\circ$ & $\circ$ & $\circ$ & \\
Yorkshire Carrots Class 1 Pack (1 kg) & $\circ$ & $\circ$ & $\circ$ & \\
\hline
\end{tabular}

\begin{tabular}{lcccc}
\hline Product name & Healthy & Unhealthy & Unsure & Calories content (100g) \\
\hline Cucumber Whole (360g) & $\circ$ & $\circ$ & $\circ$ & \\
Yorkshire Cucumber Whole (360g) & $\circ$ & $\circ$ & $\circ$ & \\
\hline
\end{tabular}

\begin{tabular}{lcccc}
\hline Product name & Healthy & Unhealthy & Unsure & Calories content (100g) \\
\hline Closed Cup Mushrooms 250g & $\circ$ & $\circ$ & $\circ$ & \\
Dorset Closed Cup Mushrooms 250g & $\circ$ & $\circ$ & $\circ$ & \\
\hline
\end{tabular}




\begin{tabular}{lcccc}
\hline Value Closed Cup Mushrooms 250g & $\circ$ & $\circ$ & $\circ$ & \\
\hline & & & & \\
& Healthy & Unhealthy & Unsure & Calories content (100g) \\
\hline Product name & $\circ$ & $\circ$ & $\circ$ & \\
\hline Tesco Frozen Petits Pois 1kg & $\circ$ & $\circ$ & $\circ$ & \\
Tesco Frozen Organic Petit Pois 500g & $\circ$ & & \\
\hline
\end{tabular}

\begin{tabular}{lcccc}
\hline Product name & Healthy & Unhealthy & Unsure & Calories content (100g) \\
\hline Baked Beans in Tomato Sauce 420g & 0 & 0 & 0 & - \\
Light Choice Baked Beans in tomato sauce 420g & $\circ$ & $\circ$ & $\circ$ & - \\
Value Baked Beans in Tomato Sauce 420g & $\circ$ & $\circ$ & $\circ$ & - \\
\hline
\end{tabular}

\begin{tabular}{lcccc}
\hline Product name & Healthy & Unhealthy & Unsure & Calories content (100g) \\
\hline Extra Virgin Olive Oil $500 \mathrm{ml}$ & $\circ$ & $\circ$ & $\circ$ & \\
Organic Extra Virgin Olive Oil $500 \mathrm{ml}$ & $\circ$ & $\circ$ & $\circ$ & \\
\hline
\end{tabular}

\begin{tabular}{lcccc}
\hline Product name & Healthy & Unhealthy & Unsure & Calories content (100g) \\
\hline Wholewheat Spaghetti 500g & 0 & 0 & $\circ$ & - \\
Value Spaghetti (500g) & $\circ$ & $\circ$ & $\circ$ & - \\
Spaghetti 500g & $\circ$ & $\circ$ & $\circ$ & - \\
Organic Spaghetti 500g & $\circ$ & $\circ$ & $\circ$ & - \\
Organic Wholewheat spaghetti 500g & $\circ$ & $\circ$ & $\circ$ & - \\
\hline
\end{tabular}

\begin{tabular}{lcccc}
\hline Product name & Healthy & Unhealthy & Unsure & Calories content (100g) \\
\hline Braised Beef \& Mash 450g & 0 & 0 & $\circ$ & - \\
Light Choice Braised Beef and Mash 450g & $\circ$ & $\circ$ & $\circ$ & - \\
Chilli con carne and rice 500g & $\circ$ & $\circ$ & $\circ$ & - \\
Light Choices Chilli Con Carne \& Rice 500g & $\circ$ & $\circ$ & $\circ$ & - \\
\hline
\end{tabular}

\begin{tabular}{lcccl}
\hline Product name & Healthy & Unhealthy & Unsure & Calories content (100g) \\
\hline Sweetened Soya milk (1 litre) & $\circ$ & $\circ$ & $\circ$ & - \\
Unsweetened Soya milk (1 litre) & $\circ$ & $\circ$ & $\circ$ & \\
Value Unweetened Soya milk (1 litre) & $\circ$ & $\circ$ & $\circ$ & \\
Organic Unsweetened Soya milk (1 litre) & $\circ$ & $\circ$ & $\circ$ & \\
Organic Sweetened Soya milk (1 litre) & $\circ$ & $\circ$ & $\circ$ & \\
\hline
\end{tabular}




\begin{tabular}{lcccc}
\hline Product name & Healthy & Unhealthy & Unsure & Calories content (100g) \\
\hline UHT value skimmed milk (1 litre) & $\circ$ & 0 & $\circ$ & \\
UHT whole milk (1 litre) & $\circ$ & $\circ$ & $\circ$ & \\
UHT skimmed milk (1 litre) & $\circ$ & $\circ$ & $\circ$ & \\
\hline
\end{tabular}

\begin{tabular}{lcccc}
\hline Product name & Healthy & Unhealthy & Unsure & Calories content (100g) \\
\hline Barn Medium eggs (6-pack) & $\circ$ & $\circ$ & $\circ$ & \\
Organic Medium eggs (6-pack) & $\circ$ & $\circ$ & $\circ$ & \\
Free-Range Medium eggs (6-pack) & $\circ$ & $\circ$ & 0 & \\
Value Medium eggs (6-pack) & $\circ$ & $\circ$ & 0 & \\
\hline
\end{tabular}

\begin{tabular}{lcccc}
\hline Product name & Healthy & Unhealthy & Unsure & Calories content (100g) \\
\hline Cola 2 I bottle & $\circ$ & $\circ$ & $\circ$ & \\
Diet Cola 2 I bottle & $\circ$ & $\circ$ & $\circ$ & \\
\hline
\end{tabular}

\begin{tabular}{lcccc}
\hline Product name & Healthy & Unhealthy & Unsure & Calories content (100g) \\
\hline English Unsalted Butter & $\circ$ & $\circ$ & $\circ$ & \\
English Salted Butter & $\circ$ & $\circ$ & $\circ$ & \\
\hline
\end{tabular}

\begin{tabular}{lcccc}
\hline Product name & Healthy & Unhealthy & Unsure & Calories content (100g) \\
\hline Cottage Pie 450g & $\circ$ & $\circ$ & $\circ$ & \\
Light Choices Cottage Pie 450g & $\circ$ & $\circ$ & $\circ$ & \\
Value Cottage Pie 450g & $\circ$ & $\circ$ & $\circ$ & \\
\hline
\end{tabular}




\section{STEP 3: Final Questions}

Intertemporal discounting (randomise order) (2 questions)

- Imagine that you receive a voucher for a free dinner at a steakhouse restaurant you like (worth £35), valid from today. Imagine also you were asked by the restaurant to wait before using this voucher, in exchange for an additional payment; how much money (in addition to the £35) would you be willing to ask as compensation to delay cashing in the voucher?

Waiting for 1 week Waiting for 2 weeks Waiting for 4 weeks

$£$

$£$

$£$

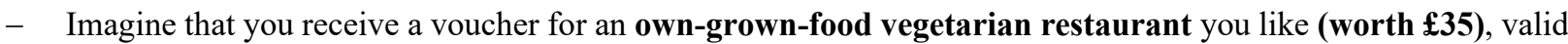
today. Imagine also you were asked by the restaurant to wait before using this voucher, in exchange for an additional payment; how much money (in addition to the £35) would you be willing to ask as compensation to delay cashing in the voucher?

Waiting for 1 week Waiting for 2 weeks Waiting for 4 weeks

$£$

$£$

Knowledge (randomise order of questions, and options within question) (4 questions)

Which one of the following has the most calories per 100 grams? (tick one)

Sugar Starchy foods Fibre roughage Fat Not sure

$\bigcirc$

○

○

O

Which do you think is higher in calories? (tick one)

$250 \mathrm{~g}$ of unsalted butter $\quad 250 \mathrm{~g}$ of regular margarine $\quad$ Both the same $\quad$ Not sure

$\begin{array}{llll}0 & 0 & 0 & 0\end{array}$

Which one of the following has the highest carbon footprint per standard mug $(250 \mathrm{ml})$ ? (tick one)
A black tea
A white coffee
A cappuccino
A latte
Not sure

○

O

o

○

$\bigcirc$ 
Which do you think is higher in carbon footprint? (tick one)

2 litres of Cola in 1 plastic bottle
2 litres of Cola in six 330-ml cans
Both the same

Not sure

Attitudes (randomise order) $(1=$ very negative, $5=$ very positive $)$

- How do you feel about actions and behaviours that protect the environment?

- How do you feel about actions and behaviours that protect one's health?

\section{Frequency of purchase}

How often have you done any of these during the last 4 weeks? (randomise order)

$\begin{array}{cccc}\text { Never 1-3 times } & \text { 1-2 times } & \text { 3-6 times } & \begin{array}{l}\text { More often than } 6 \\ \text { times per week }\end{array} \\ & \text { per month } & \text { per week } & \text { per week }\end{array}$

Purchased food with a health label (e.g., low fat; low salt) to keep healthy.

Purchased food with an environmentally-friendly

○

○

○

O

label to protect the environment.

Self-identity $(1=$ totally disagree; $5=$ totally agree) (randomise order)

Please answer your level of agreement to each statement, which refer to the adjective on top of it.

- Being environmentally-friendly is an important part of who I am.

- Being healthy is an important part of who I am. 
ONLINE APPENDIX 2: Labels used in the "labelling" condition

\section{Carbon label}

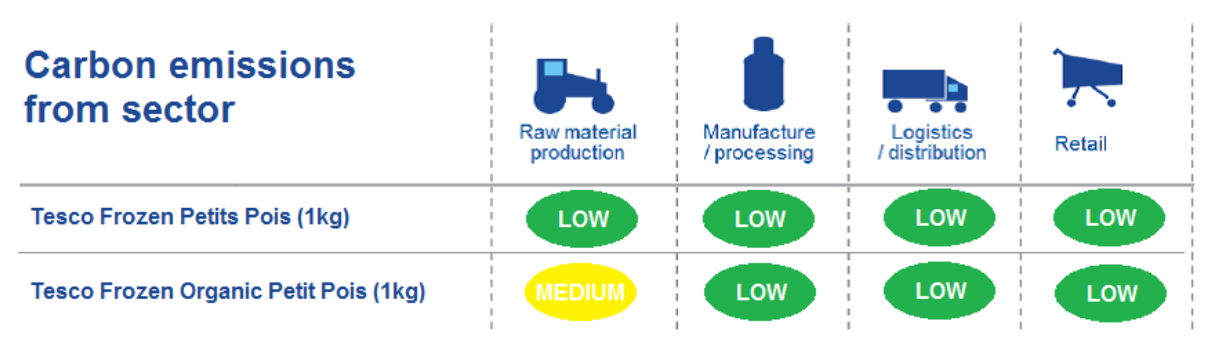

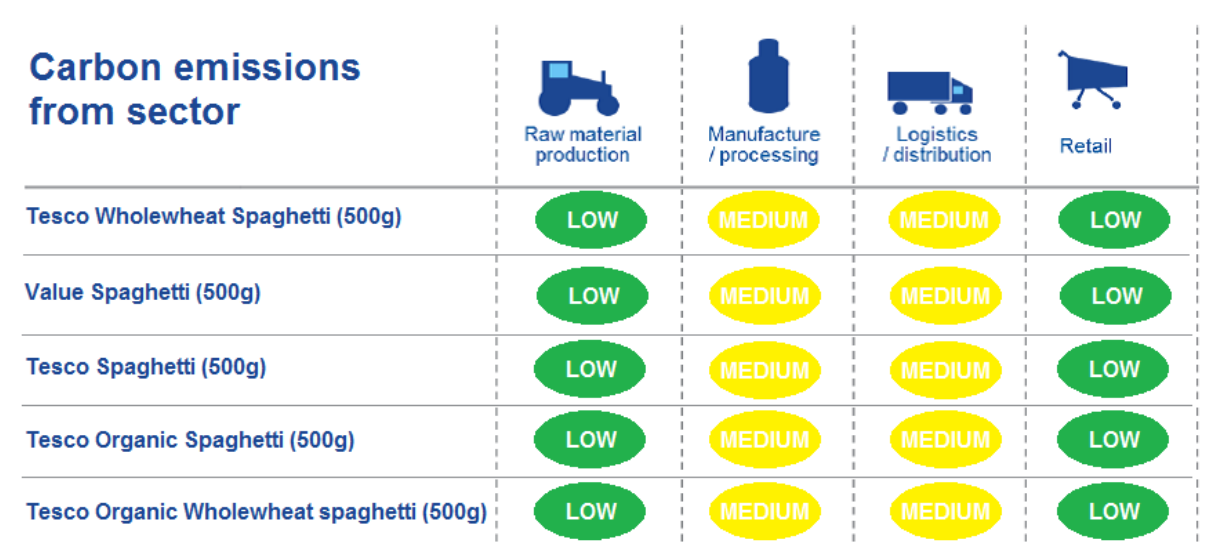

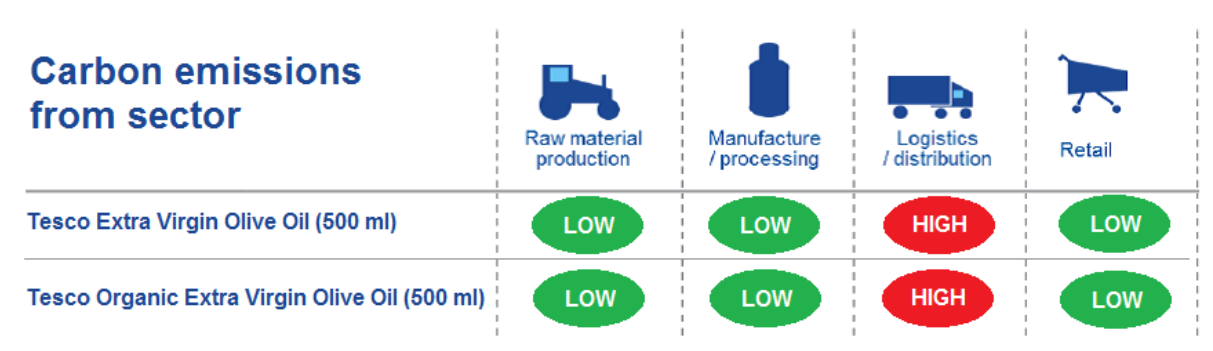

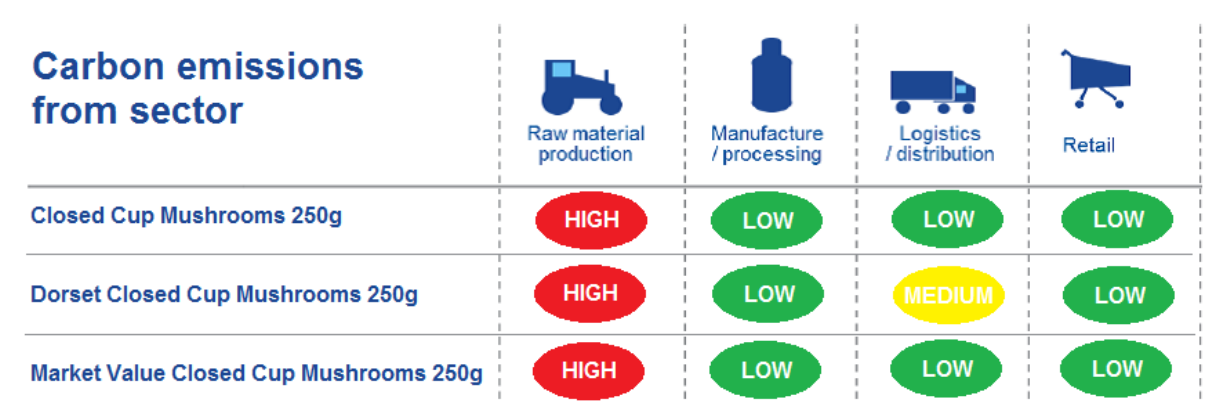






\begin{tabular}{l|l|l|l|l|}
$\begin{array}{l}\text { Carbon emissions } \\
\text { from sector }\end{array}$ & $\begin{array}{c}\text { Raw material } \\
\text { production }\end{array}$ & $\begin{array}{c}\text { Manufacture } \\
\text { /processing }\end{array}$ & $\begin{array}{c}\text { Logistics } \\
\text { /distribution }\end{array}$ & Retail \\
\hline Barn eggs Large (6-pack) & MEDIUn & LOW & LOW & LOW \\
\hline Organic Large eggs (6-pack) & HIGH & LOW & LOW & LOW \\
\hline Free-Range Large eggs (6-pack) & MEIUW & LOW & LOW & LOW \\
\hline Value eggs (6-pack) & HIGH & LOW & LOW & LOW \\
\hline
\end{tabular}

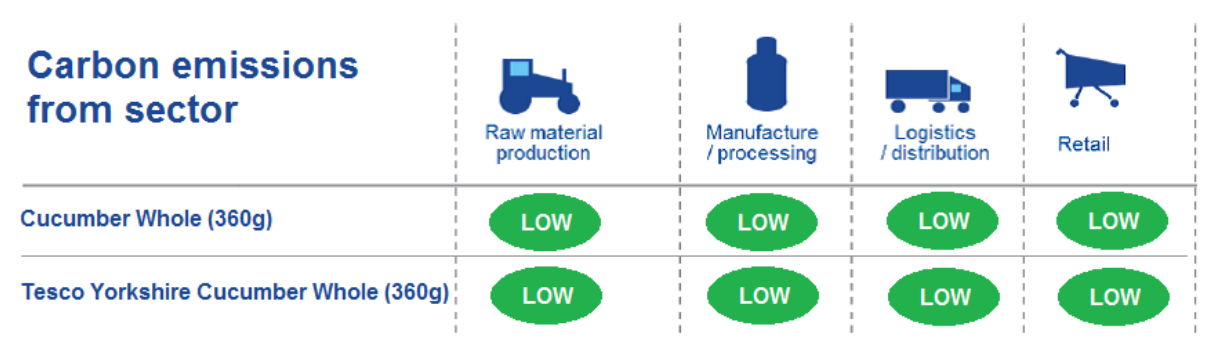

\begin{tabular}{|c|c|c|c|c|}
\hline $\begin{array}{l}\text { Carbon emissions } \\
\text { from sector }\end{array}$ & & $\begin{array}{l}\text { Manufacture } \\
\text { /processing }\end{array}$ & $\underset{\substack{\text { Logistics } \\
\text { / distribution }}}{\text { בר. }}$ & Retail \\
\hline Cottage Pie (450g) &  & & MEDIUM & MEDIUM \\
\hline Light Choices Cottage Pie (450g) & Ium & & MEDIUM & MEDIUM \\
\hline Value Cottage Pie (450g) & MEDIUM & & MEDIUM & MEDIUM \\
\hline
\end{tabular}

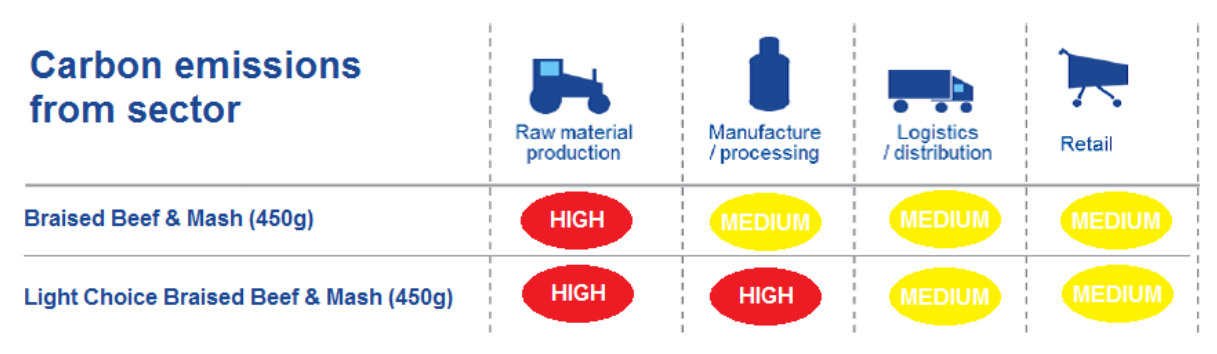



\begin{tabular}{l|c|c|c|c|}
$\begin{array}{l}\text { Carbon emissions } \\
\text { from sector }\end{array}$ & $\begin{array}{c}\text { Raw material } \\
\text { production }\end{array}$ & $\begin{array}{c}\text { Manufacture } \\
\text { /processing }\end{array}$ & $\begin{array}{c}\text { Logistics } \\
\text { /distribution }\end{array}$ & Retail \\
\hline Cola 2 bottle & LEDIUW & LOW & LOW & LOW \\
\hline Diet Cola 2 I bottle & LOW & LOW & LOW & LOW
\end{tabular}




\begin{tabular}{|c|c|c|c|c|}
\hline $\begin{array}{l}\text { Carbon emissions } \\
\text { from sector }\end{array}$ & $\begin{array}{l}\text { Raw material } \\
\text { production }\end{array}$ & $\begin{array}{l}\text { Manufacture } \\
\text { / processing }\end{array}$ & $\begin{array}{l}\text { Logistics } \\
\text { / distribution }\end{array}$ & \\
\hline Carrots Class 1 Pack (1 kg) & & w & Low & \\
\hline Market Value Carrots 1 Pack (1 kg) & & & on & \\
\hline kshire Carrots Class 1 Pack (1 ko & & & & \\
\hline
\end{tabular}

\begin{tabular}{|c|c|c|c|c|}
\hline $\begin{array}{l}\text { Carbon emissions } \\
\text { from sector }\end{array}$ & $\underset{\substack{\text { Raw material } \\
\text { production }}}{ }$ & Manufacture & $\begin{array}{l}\text { Logistics } \\
\text { / distribution }\end{array}$ & Retail \\
\hline English Unsalted Butter & MEDIUM & & LO & \\
\hline English Salted Butter & & & & \\
\hline
\end{tabular}

\begin{tabular}{l|c|c|c|c|}
$\begin{array}{l}\text { Carbon emissions } \\
\text { from sector }\end{array}$ & $\begin{array}{l}\text { Raw material } \\
\text { production }\end{array}$ & $\begin{array}{c}\text { Manufacture } \\
\text { /processing }\end{array}$ & $\begin{array}{l}\text { L Logistics } \\
\text { /distribution }\end{array}$ & Retail \\
\hline Tesco Baked Beans in tomato sauce (420g) & LOW & LOW & LOW & LOW \\
\hline $\begin{array}{l}\text { Tesco Light Choice Baked Beans in tomato } \\
\text { sauce (420g) }\end{array}$ & LOW & LOW & LOW & LOW \\
$\begin{array}{l}\text { Tesco Value Baked Beans in tomato sauce } \\
\text { (420g) }\end{array}$ & LOW & LOW & LOW & LOW \\
\end{tabular}

\begin{tabular}{|c|c|c|c|c|}
\hline $\begin{array}{l}\text { Carbon emissions } \\
\text { from sector }\end{array}$ & $\begin{array}{c}\text { Raw material } \\
\text { production }\end{array}$ & $\begin{array}{l}\text { Manufacture } \\
\text { / processing }\end{array}$ & $\begin{array}{l}\text { Logistics } \\
\text { / distribution }\end{array}$ & Retail \\
\hline Sweetened Soya milk (1 I) & tento & & MEDIUM & MEDIUM \\
\hline Unsweetened Soya milk (1 I) & & & MEDIUM & MEDIUM \\
\hline Value Unsweetened Soya milk (1 I) & & & MEDIUM & MEDIUM \\
\hline Organic Unsweetened Soya milk (1 I) & EDIUM & & MEDIUM & MEDIUM \\
\hline Organic Sweetened Soya milk (1 I) & 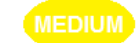 & & MEDIUM & MEDIUM \\
\hline
\end{tabular}




\section{Nutrition label}

\begin{tabular}{l|c|c|c} 
Nutrient content (100 g) & $\begin{array}{l}\text { Carbohydrates } \\
\text { (incl. sugars) }\end{array}$ & Fats & Salt \\
\hline Sweetened Soya milk (1 I) & $2.6 \mathrm{~g}$ & $1.9 \mathrm{~g}$ & $0.2 \mathrm{~g}$ \\
\hline Unsweetened Soya milk (1) & $0.4 \mathrm{~g}$ & $1.9 \mathrm{~g}$ & $0.1 \mathrm{~g}$ \\
\hline Value Unsweetened Soya milk (1 I) & $0.3 \mathrm{~g}$ & $1.9 \mathrm{~g}$ & $0.1 \mathrm{~g}$ \\
\hline Organic Unsweetened Soya milk (1) & $0.4 \mathrm{~g}$ & $1.9 \mathrm{~g}$ & $0.1 \mathrm{~g}$ \\
\hline Organic Sweetened Soya milk (1 I) & $2.4 \mathrm{~g}$ & $1.9 \mathrm{~g}$ & $0.2 \mathrm{~g}$ \\
\hline
\end{tabular}

\begin{tabular}{l|c|c|c} 
Nutrient content (100 g) & $\begin{array}{l}\text { Carbohydrates } \\
\text { (incl. sugars) }\end{array}$ & Fats & Salt \\
\hline Tesco Frozen Petits Pois (1kg) & $5.5 \mathrm{~g}$ & $0.9 \mathrm{~g}$ & $0 \mathrm{~g}$ \\
\hline Tesco Frozen Organic Petit Pois (1kg) & $5.5 \mathrm{~g}$ & $0.9 \mathrm{~g}$ & $0 \mathrm{~g}$ \\
\hline
\end{tabular}

\begin{tabular}{|c|c|c|c|}
\hline Nutrient content (100 g) & $\begin{array}{l}\text { Carbohydrates } \\
\text { (incl. sugars) }\end{array}$ & Fats & Salt \\
\hline \multicolumn{4}{|l|}{ Tesco Wholewheat Spaghetti (500g) } \\
\hline \multicolumn{4}{|l|}{ Value Spaghetti $(500 \mathrm{~g})$} \\
\hline \multicolumn{4}{|l|}{ Tesco Spaghetti $(500 \mathrm{~g})$} \\
\hline \multicolumn{4}{|l|}{ Tesco Organic Spaghetti $(500 \mathrm{~g})$} \\
\hline \multicolumn{4}{|l|}{ Tesco Organic Wholewheat spaghetti $(500 \mathrm{~g})$} \\
\hline Nutrient content (100 g) & $\begin{array}{l}\text { Carbohydrates } \\
\text { (incl. sugars) }\end{array}$ & Fats & Salt \\
\hline \multicolumn{4}{|l|}{ Tesco Extra Virgin Olive Oil (500 ml) } \\
\hline Tesco Organic Extra Virgin Olive Oil $(500 \mathrm{ml}$ & & & $0 \mathrm{~g}$ \\
\hline
\end{tabular}

\begin{tabular}{l|l|l|l} 
Nutrient content (100 g) & $\begin{array}{l}\text { Carbohydrates } \\
\text { (incl. sugars) }\end{array}$ & Fats & Salt \\
\hline Closed Cup Mushrooms 250g & $0.4 \mathrm{~g}$ & $0.5 \mathrm{~g}$ & $0.1 \mathrm{~g}$ \\
\hline Dorset Closed Cup Mushrooms 250g & $0.4 \mathrm{~g}$ & $0.5 \mathrm{~g}$ & $0.1 \mathrm{~g}$ \\
\hline Market Value Closed Cup Mushrooms 250g & $0.4 \mathrm{~g}$ & $0.5 \mathrm{~g}$ & $0.1 \mathrm{~g}$ \\
\hline
\end{tabular}




\begin{tabular}{|c|c|c|c|}
\hline Nutrient content (100 g) & $\begin{array}{l}\text { Carbohydrates } \\
\text { (incl. sugars) }\end{array}$ & Fats & Salt \\
\hline \multicolumn{4}{|l|}{ UHT Value Skimmed milk (1 I) } \\
\hline \multicolumn{4}{|l|}{ UHT Whole milk (1 I) } \\
\hline \multicolumn{4}{|l|}{ UHT Skimmed milk (1 I) } \\
\hline Nutrient content (100 g) & $\begin{array}{l}\text { Carbohydrates } \\
\text { (incl. sugars) }\end{array}$ & Fats & Salt \\
\hline \multicolumn{4}{|l|}{ Barn Medium eggs (6-pack) } \\
\hline \multicolumn{4}{|l|}{ Organic Medium eggs (6-pack) } \\
\hline \multicolumn{4}{|l|}{ Free-Range Medium eggs (6-pack) } \\
\hline \multicolumn{4}{|l|}{ Value Medium eggs (6-pack) } \\
\hline Nutrient content (100 g) & $\begin{array}{l}\text { Carbohydrates } \\
\text { (incl. sugars) }\end{array}$ & Fats & Salt \\
\hline \multicolumn{4}{|l|}{ Cucumber Whole $(360 \mathrm{~g})$} \\
\hline \multicolumn{4}{|l|}{ Yorkshire Cucumber Whole $(360 \mathrm{~g})$} \\
\hline Nutrient content (100 g) & $\begin{array}{l}\text { Carbohydrates } \\
\text { (incl. sugars) }\end{array}$ & Fats & Salt \\
\hline \multicolumn{4}{|l|}{ Cottage Pie $(450 \mathrm{~g})$} \\
\hline \multicolumn{4}{|l|}{ Light Choices Cottage Pie $(450 \mathrm{~g})$} \\
\hline Value Cottage Pie $(450 \mathrm{~g})$ & & & 0.4 \\
\hline
\end{tabular}

\begin{tabular}{l|c|c|c} 
Nutrient content $(\mathbf{1 0 0} \mathbf{~ g})$ & $\begin{array}{l}\text { Carbohydrates } \\
\text { (incl. sugars) }\end{array}$ & Fats & Salt \\
\hline Cola 2 I bottle & $9.7 \mathrm{~g}$ & $0 \mathrm{~g}$ & $0 \mathrm{~g}$ \\
\hline Diet Cola 2 I bottle & $0.1 \mathrm{~g}$ & $0 \mathrm{~g}$ & $0.1 \mathrm{~g}$
\end{tabular}

\begin{tabular}{l|l|l|l} 
Nutrient content (100 g) & $\begin{array}{l}\text { Carbohydrates } \\
\text { (incl. sugars) }\end{array}$ & Fats & Salt \\
\hline Chilli con carne and rice (450g) & $15.4 \mathrm{~g}$ & $2.4 \mathrm{~g}$ & $0.5 \mathrm{~g}$ \\
\hline Light Choices Chilli Con Carne \& Rice (450g) & $\mathbf{1 5 . 8 \mathrm { g }}$ & $1.6 \mathrm{~g}$ & $0.3 \mathrm{~g}$
\end{tabular}




\begin{tabular}{|c|c|c|c|}
\hline Nutrient content (100 g) & $\begin{array}{l}\text { Carbohydrates } \\
\text { (incl. sugars) }\end{array}$ & Fats & Salt \\
\hline \multicolumn{4}{|l|}{ Carrots Class 1 Pack (1 kg) } \\
\hline \multicolumn{4}{|l|}{ Market Value Carrots 1 Pack (1 kg) } \\
\hline \multicolumn{4}{|l|}{ Yorkshire Carrots Class 1 Pack (1 kg) } \\
\hline Nutrient content (100 g) & $\begin{array}{l}\text { Carbohydrates } \\
\text { (incl. sugars) }\end{array}$ & Fats & Salt \\
\hline \multicolumn{4}{|l|}{ English Unsalted Butter } \\
\hline \multicolumn{4}{|l|}{ English Salted Butter } \\
\hline Nutrient content (100 g) & $\begin{array}{l}\text { Carbohydrates } \\
\text { (incl. sugars) }\end{array}$ & Fats & Salt \\
\hline \multicolumn{4}{|l|}{ Braised Beef \& Mash (450g) } \\
\hline \multicolumn{4}{|l|}{ Light Choice Braised Beef \& Mash (450g) } \\
\hline Nutrient content (100 g) & $\begin{array}{l}\text { Carbohydrates } \\
\text { (incl. sugars) }\end{array}$ & Fats & Salt \\
\hline \multicolumn{4}{|l|}{ Tesco Baked Beans in tomato sauce $(420 \mathrm{~g})$} \\
\hline \multicolumn{4}{|l|}{$\begin{array}{l}\text { Tesco Light Choice Baked Beans in tomato } \\
\text { sauce }(420 \mathrm{~g})\end{array}$} \\
\hline $\begin{array}{l}\text { Tesco Value Baked Beans in tomato sauce } \\
(420 \mathrm{~g})\end{array}$ & & & \\
\hline
\end{tabular}


ONLINE APPENDIX 3: Mean and the $95 \%$ confidence interval of a metric for each product

a) Carbon footprint

\begin{tabular}{|c|c|c|c|c|c|c|c|c|c|c|c|c|}
\hline \multirow{3}{*}{$\begin{array}{l}\text { Product name } \\
\text { Carrots Class } 1 \text { Pack }(1 \mathrm{~kg})\end{array}$} & \multicolumn{3}{|c|}{ Control } & \multicolumn{3}{|c|}{ Time pressure } & \multicolumn{3}{|c|}{ Labelling } & \multicolumn{3}{|c|}{ Labelling + Time pressure } \\
\hline & \multirow{2}{*}{$\begin{array}{l}\text { Mean } \\
4.65\end{array}$} & \multicolumn{2}{|c|}{$95 \%$ conf. int. } & \multirow{2}{*}{$\begin{array}{l}\text { Mean } \\
4.62\end{array}$} & \multicolumn{2}{|c|}{$95 \%$ conf. int. } & \multirow{2}{*}{$\begin{array}{l}\text { Mean } \\
4.22\end{array}$} & \multicolumn{2}{|c|}{$95 \%$ conf. int. } & \multirow{2}{*}{$\begin{array}{l}\text { Mean } \\
4.18\end{array}$} & \multicolumn{2}{|c|}{$95 \%$ conf. int. } \\
\hline & & 4.54 & 4.54 & & 4.50 & 4.50 & & 4.10 & 4.10 & & 4.04 & 4.04 \\
\hline Market Value Carrots 1 Pack (1 kg) & 4.68 & 4.56 & 4.56 & 4.65 & 4.52 & 4.52 & 4.24 & 4.12 & 4.12 & 4.17 & 4.03 & 4.03 \\
\hline Yorkshire Carrots Class 1 Pack (1 kg) & 4.62 & 4.50 & 4.50 & 4.55 & 4.43 & 4.43 & 4.21 & 4.09 & 4.09 & 4.17 & 4.03 & 4.03 \\
\hline Cucumber Whole (360g) & 4.39 & 4.26 & 4.26 & 4.38 & 4.25 & 4.25 & 3.99 & 3.86 & 3.86 & 4.02 & 3.88 & 3.88 \\
\hline Yorkshire Cucumber Whole (360g) & 4.33 & 4.20 & 4.20 & 4.30 & 4.16 & 4.16 & 3.98 & 3.85 & 3.85 & 4.02 & 3.88 & 3.88 \\
\hline Closed Cup Mushrooms 250g & 4.56 & 4.43 & 4.43 & 4.62 & 4.50 & 4.50 & 5.09 & 4.97 & 4.97 & 5.05 & 4.93 & 4.93 \\
\hline Dorset Closed Cup Mushrooms 250g & 4.54 & 4.42 & 4.42 & 4.60 & 4.49 & 4.49 & 5.18 & 5.07 & 5.07 & 5.10 & 4.98 & 4.98 \\
\hline Value Closed Cup Mushrooms 250g & 4.65 & 4.53 & 4.53 & 4.66 & 4.54 & 4.54 & 5.10 & 4.98 & 4.98 & 5.07 & 4.95 & 4.95 \\
\hline Frozen Petits Pois $1 \mathrm{~kg}$ & 5.10 & 4.98 & 4.98 & 5.03 & 4.92 & 4.92 & 4.62 & 4.50 & 4.50 & 4.60 & 4.46 & 4.46 \\
\hline Frozen Organic Petit Pois 1kg & 5.05 & 4.93 & 4.93 & 4.99 & 4.87 & 4.87 & 4.74 & 4.62 & 4.62 & 4.69 & 4.56 & 4.56 \\
\hline Baked Beans in Tomato Sauce 420g & 5.53 & 5.43 & 5.43 & 5.51 & 5.42 & 5.42 & 4.90 & 4.79 & 4.79 & 4.97 & 4.84 & 4.84 \\
\hline Light Choice Baked Beans in tomato sauce $420 \mathrm{~g}$ & 5.52 & 5.42 & 5.42 & 5.50 & 5.40 & 5.40 & 4.90 & 4.79 & 4.79 & 4.96 & 4.83 & 4.83 \\
\hline Value Baked Beans in Tomato Sauce $420 \mathrm{~g}$ & 5.53 & 5.44 & 5.44 & 5.55 & 5.46 & 5.46 & 4.94 & 4.83 & 4.83 & 4.96 & 4.83 & 4.83 \\
\hline Extra Virgin Olive Oil $500 \mathrm{ml}$ & 5.38 & 5.25 & 5.25 & 5.40 & 5.28 & 5.28 & 5.39 & 5.28 & 5.28 & 5.32 & 5.19 & 5.19 \\
\hline Organic Extra Virgin Olive Oil $500 \mathrm{ml}$ & 5.32 & 5.18 & 5.18 & 5.37 & 5.25 & 5.25 & 5.39 & 5.28 & 5.28 & 5.32 & 5.19 & 5.19 \\
\hline Whole wheat Spaghetti 500g & 5.39 & 5.28 & 5.28 & 5.36 & 5.25 & 5.25 & 5.32 & 5.22 & 5.22 & 5.29 & 5.17 & 5.17 \\
\hline Value Spaghetti (500g) & 5.45 & 5.33 & 5.33 & 5.46 & 5.35 & 5.35 & 5.34 & 5.24 & 5.24 & 5.34 & 5.22 & 5.22 \\
\hline Spaghetti 500g & 5.44 & 5.33 & 5.33 & 5.42 & 5.32 & 5.32 & 5.34 & 5.23 & 5.23 & 5.32 & 5.20 & 5.20 \\
\hline Organic Spaghetti 500g & 5.33 & 5.21 & 5.21 & 5.34 & 5.23 & 5.23 & 5.32 & 5.21 & 5.21 & 5.28 & 5.16 & 5.16 \\
\hline Organic Whole wheat spaghetti 500g & 5.30 & 5.17 & 5.17 & 5.30 & 5.18 & 5.18 & 5.29 & 5.18 & 5.18 & 5.27 & 5.15 & 5.15 \\
\hline Braised Beef \& Mash 450g & 6.19 & 6.08 & 6.08 & 6.08 & 5.97 & 5.97 & 6.28 & 6.17 & 6.17 & 6.09 & 5.96 & 5.96 \\
\hline Light Choice Braised Beef and Mash 450g & 6.13 & 6.02 & 6.02 & 6.03 & 5.92 & 5.92 & 6.23 & 6.12 & 6.12 & 6.05 & 5.92 & 5.92 \\
\hline Chilli con carne and rice $500 \mathrm{~g}$ & 6.18 & 6.07 & 6.07 & 6.09 & 5.98 & 5.98 & 6.24 & 6.14 & 6.14 & 6.06 & 5.93 & 5.93 \\
\hline Light Choices Chilli Con Carne \& Rice 500g & 6.14 & 6.03 & 6.03 & 6.07 & 5.96 & 5.96 & 6.28 & 6.18 & 6.18 & 6.09 & 5.97 & 5.97 \\
\hline Sweetened Soya milk (1 litre) & 5.36 & 5.24 & 5.24 & 5.34 & 5.22 & 5.22 & 5.72 & 5.60 & 5.60 & 5.60 & 5.47 & 5.47 \\
\hline Unsweetened Soya milk (1 litre) & 5.23 & 5.10 & 5.10 & 5.23 & 5.11 & 5.11 & 5.61 & 5.49 & 5.49 & 5.51 & 5.38 & 5.38 \\
\hline Value Unsweetened Soya milk (1 litre) & 5.30 & 5.17 & 5.17 & 5.27 & 5.15 & 5.15 & 5.61 & 5.50 & 5.50 & 5.52 & 5.39 & 5.39 \\
\hline
\end{tabular}




\begin{tabular}{|c|c|c|c|c|c|c|c|c|c|c|c|c|}
\hline Organic Unsweetened Soya milk (1 litre) & 5.22 & 5.10 & 5.10 & 5.21 & 5.10 & 5.10 & 5.69 & 5.57 & 5.57 & 5.55 & 5.42 & 5.42 \\
\hline Organic Sweetened Soya milk (1 litre) & 5.31 & 5.19 & 5.19 & 5.30 & 5.18 & 5.18 & 5.71 & 5.60 & 5.60 & 5.58 & 5.45 & 5.45 \\
\hline UHT value skimmed milk (1 litre) & 5.34 & 5.22 & 5.22 & 5.31 & 5.19 & 5.19 & 5.09 & 4.97 & 4.97 & 5.12 & 4.99 & 4.99 \\
\hline UHT whole milk (1 litre) & 5.40 & 5.28 & 5.28 & 5.30 & 5.17 & 5.17 & 5.20 & 5.08 & 5.08 & 5.20 & 5.07 & 5.07 \\
\hline UHT skimmed milk (1 litre) & 5.32 & 5.20 & 5.20 & 5.29 & 5.17 & 5.17 & 5.09 & 4.97 & 4.97 & 5.11 & 4.98 & 4.98 \\
\hline Barn Medium eggs (6-pack) & 5.33 & 5.21 & 5.21 & 5.23 & 5.12 & 5.12 & 5.19 & 5.08 & 5.08 & 5.06 & 4.93 & 4.93 \\
\hline Organic Medium eggs (6-pack) & 5.22 & 5.11 & 5.11 & 5.11 & 5.00 & 5.00 & 5.30 & 5.18 & 5.18 & 5.15 & 5.01 & 5.01 \\
\hline Free-Range Medium eggs (6-pack) & 5.15 & 5.03 & 5.03 & 5.05 & 4.95 & 4.95 & 5.15 & 5.04 & 5.04 & 4.98 & 4.84 & 4.84 \\
\hline Value Medium eggs (6-pack) & 5.39 & 5.27 & 5.27 & 5.31 & 5.20 & 5.20 & 5.35 & 5.24 & 5.24 & 5.24 & 5.11 & 5.11 \\
\hline Cola 2 I bottle & 5.71 & 5.59 & 5.59 & 5.81 & 5.70 & 5.70 & 4.97 & 4.86 & 4.86 & 5.16 & 5.03 & 5.03 \\
\hline Diet Cola 21 bottle & 5.65 & 5.52 & 5.52 & 5.79 & 5.69 & 5.69 & 4.77 & 4.65 & 4.65 & 4.99 & 4.84 & 4.84 \\
\hline English Unsalted Butter & 5.43 & 5.32 & 5.32 & 5.34 & 5.22 & 5.22 & 5.78 & 5.68 & 5.68 & 5.72 & 5.60 & 5.60 \\
\hline English Salted Butter & 5.49 & 5.38 & 5.38 & 5.37 & 5.26 & 5.26 & 5.80 & 5.71 & 5.71 & 5.74 & 5.62 & 5.62 \\
\hline Cottage Pie 450g & 6.08 & 5.96 & 5.96 & 5.98 & 5.87 & 5.87 & 6.26 & 6.15 & 6.15 & 6.14 & 6.03 & 6.03 \\
\hline Light Choices Cottage Pie 450g & 6.03 & 5.91 & 5.91 & 5.96 & 5.85 & 5.85 & 6.19 & 6.09 & 6.09 & 6.01 & 5.90 & 5.90 \\
\hline Value Cottage Pie 450g & 6.08 & 5.95 & 5.95 & 6.00 & 5.89 & 5.89 & 6.19 & 6.09 & 6.09 & 6.01 & 5.89 & 5.89 \\
\hline
\end{tabular}

b) kilocalories

\begin{tabular}{|c|c|c|c|c|c|c|c|c|c|c|c|c|}
\hline \multirow{3}{*}{$\begin{array}{l}\text { Product name } \\
\text { Carrots Class } 1 \text { Pack (1 kg) }\end{array}$} & \multicolumn{3}{|c|}{ Control } & \multicolumn{3}{|c|}{ Time pressure } & \multicolumn{3}{|c|}{ Labelling } & \multicolumn{3}{|c|}{ Labelling + Time pressure } \\
\hline & \multirow{2}{*}{$\begin{array}{l}\text { Mean } \\
3.87\end{array}$} & \multicolumn{2}{|c|}{$95 \%$ conf. int. } & \multirow{2}{*}{$\begin{array}{l}\text { Mean } \\
3.91\end{array}$} & \multicolumn{2}{|c|}{$95 \%$ conf. int. } & \multirow{2}{*}{$\begin{array}{l}\text { Mean } \\
3.73\end{array}$} & \multicolumn{2}{|c|}{$95 \%$ conf. int. } & \multirow{2}{*}{$\begin{array}{l}\text { Mean } \\
3.82\end{array}$} & \multicolumn{2}{|c|}{$95 \%$ conf. int. } \\
\hline & & 3.76 & 3.76 & & 3.80 & 3.80 & & 3.61 & 3.61 & & 3.70 & 3.70 \\
\hline Market Value Carrots 1 Pack (1 kg) & 3.89 & 3.77 & 3.77 & 3.93 & 3.82 & 3.82 & 3.73 & 3.62 & 3.62 & 3.83 & 3.71 & 3.71 \\
\hline Yorkshire Carrots Class 1 Pack (1 kg) & 3.88 & 3.76 & 3.76 & 3.93 & 3.82 & 3.82 & 3.73 & 3.62 & 3.62 & 3.82 & 3.70 & 3.70 \\
\hline Cucumber Whole (360g) & 3.16 & 3.04 & 3.04 & 3.27 & 3.15 & 3.15 & 3.01 & 2.90 & 2.90 & 3.18 & 3.05 & 3.05 \\
\hline Yorkshire Cucumber Whole (360g) & 3.16 & 3.04 & 3.04 & 3.29 & 3.17 & 3.17 & 3.01 & 2.90 & 2.90 & 3.22 & 3.09 & 3.09 \\
\hline Closed Cup Mushrooms 250g & 3.71 & 3.60 & 3.60 & 3.77 & 3.65 & 3.65 & 3.53 & 3.42 & 3.42 & 3.67 & 3.55 & 3.55 \\
\hline Dorset Closed Cup Mushrooms 250g & 3.71 & 3.61 & 3.61 & 3.78 & 3.67 & 3.67 & 3.52 & 3.41 & 3.41 & 3.68 & 3.56 & 3.56 \\
\hline Value Closed Cup Mushrooms 250g & 3.72 & 3.62 & 3.62 & 3.79 & 3.68 & 3.68 & 3.52 & 3.41 & 3.41 & 3.68 & 3.56 & 3.56 \\
\hline Frozen Petits Pois $1 \mathrm{~kg}$ & 4.14 & 4.03 & 4.03 & 4.11 & 4.00 & 4.00 & 4.00 & 3.90 & 3.90 & 4.11 & 4.00 & 4.00 \\
\hline Frozen Organic Petit Pois $1 \mathrm{~kg}$ & 4.07 & 3.96 & 3.96 & 4.09 & 3.98 & 3.98 & 3.96 & 3.86 & 3.86 & 4.08 & 3.97 & 3.97 \\
\hline Baked Beans in Tomato Sauce $420 \mathrm{~g}$ & 4.96 & 4.87 & 4.87 & 5.00 & 4.92 & 4.92 & 4.85 & 4.77 & 4.77 & 4.93 & 4.84 & 4.84 \\
\hline Light Choice Baked Beans in tomato sauce $420 \mathrm{~g}$ & 4.76 & 4.68 & 4.68 & 4.82 & 4.73 & 4.73 & 4.68 & 4.60 & 4.60 & 4.78 & 4.69 & 4.69 \\
\hline Value Baked Beans in Tomato Sauce $420 \mathrm{~g}$ & 4.97 & 4.89 & 4.89 & 5.03 & 4.95 & 4.95 & 4.85 & 4.76 & 4.76 & 4.93 & 4.84 & 4.84 \\
\hline
\end{tabular}




\begin{tabular}{|c|c|c|c|c|c|c|c|c|c|c|c|c|}
\hline Extra Virgin Olive Oil $500 \mathrm{ml}$ & 5.22 & 5.10 & 5.10 & 5.03 & 4.90 & 4.90 & 5.31 & 5.19 & 5.19 & 5.20 & 5.08 & 5.08 \\
\hline Organic Extra Virgin Olive Oil $500 \mathrm{ml}$ & 5.22 & 5.10 & 5.10 & 5.02 & 4.89 & 4.89 & 5.32 & 5.20 & 5.20 & 5.19 & 5.07 & 5.07 \\
\hline Whole wheat Spaghetti 500g & 4.95 & 4.86 & 4.86 & 4.91 & 4.83 & 4.83 & 5.03 & 4.95 & 4.95 & 5.03 & 4.93 & 4.93 \\
\hline Value Spaghetti (500g) & 5.06 & 4.97 & 4.97 & 5.04 & 4.95 & 4.95 & 5.10 & 5.01 & 5.01 & 5.09 & 4.99 & 4.99 \\
\hline Spaghetti 500g & 5.05 & 4.96 & 4.96 & 5.03 & 4.95 & 4.95 & 5.11 & 5.02 & 5.02 & 5.10 & 5.00 & 5.00 \\
\hline Organic Spaghetti 500g & 4.99 & 4.90 & 4.90 & 5.00 & 4.91 & 4.91 & 5.07 & 4.98 & 4.98 & 5.05 & 4.95 & 4.95 \\
\hline Organic Whole wheat spaghetti 500g & 4.93 & 4.84 & 4.84 & 4.92 & 4.83 & 4.83 & 5.05 & 4.96 & 4.96 & 5.02 & 4.92 & 4.92 \\
\hline Braised Beef \& Mash 450g & 5.67 & 5.58 & 5.58 & 5.65 & 5.56 & 5.56 & 5.69 & 5.60 & 5.60 & 5.77 & 5.68 & 5.68 \\
\hline Light Choice Braised Beef and Mash 450g & 5.47 & 5.38 & 5.38 & 5.47 & 5.39 & 5.39 & 5.53 & 5.45 & 5.45 & 5.63 & 5.54 & 5.54 \\
\hline Chilli con carne and rice $500 \mathrm{~g}$ & 5.74 & 5.66 & 5.66 & 5.71 & 5.62 & 5.62 & 5.66 & 5.58 & 5.58 & 5.79 & 5.71 & 5.71 \\
\hline Light Choices Chilli Con Carne \& Rice 500g & 5.57 & 5.48 & 5.48 & 5.54 & 5.45 & 5.45 & 5.52 & 5.44 & 5.44 & 5.67 & 5.59 & 5.59 \\
\hline Sweetened Soya milk (1 litre) & 4.80 & 4.69 & 4.69 & 4.79 & 4.69 & 4.69 & 4.55 & 4.44 & 4.44 & 4.68 & 4.56 & 4.56 \\
\hline Unsweetened Soya milk (1 litre) & 4.44 & 4.33 & 4.33 & 4.46 & 4.36 & 4.36 & 4.14 & 4.02 & 4.02 & 4.32 & 4.20 & 4.20 \\
\hline Value Unsweetened Soya milk (1 litre) & 4.48 & 4.37 & 4.37 & 4.50 & 4.40 & 4.40 & 4.15 & 4.04 & 4.04 & 4.37 & 4.25 & 4.25 \\
\hline Organic Unsweetened Soya milk (1 litre) & 4.44 & 4.34 & 4.34 & 4.48 & 4.38 & 4.38 & 4.13 & 4.02 & 4.02 & 4.30 & 4.17 & 4.17 \\
\hline Organic Sweetened Soya milk (1 litre) & 4.76 & 4.65 & 4.65 & 4.75 & 4.65 & 4.65 & 4.46 & 4.35 & 4.35 & 4.60 & 4.48 & 4.48 \\
\hline UHT value skimmed milk (1 litre) & 4.33 & 4.22 & 4.22 & 4.35 & 4.24 & 4.24 & 4.36 & 4.26 & 4.26 & 4.53 & 4.42 & 4.42 \\
\hline UHT whole milk (1 litre) & 4.84 & 4.74 & 4.74 & 4.82 & 4.71 & 4.71 & 4.79 & 4.69 & 4.69 & 4.96 & 4.85 & 4.85 \\
\hline UHT skimmed milk (1 litre) & 4.32 & 4.20 & 4.20 & 4.33 & 4.22 & 4.22 & 4.36 & 4.25 & 4.25 & 4.54 & 4.42 & 4.42 \\
\hline Barn Medium eggs (6-pack) & 4.74 & 4.63 & 4.63 & 4.68 & 4.57 & 4.57 & 4.76 & 4.67 & 4.67 & 4.80 & 4.69 & 4.69 \\
\hline Organic Medium eggs (6-pack) & 4.71 & 4.61 & 4.61 & 4.67 & 4.57 & 4.57 & 4.73 & 4.64 & 4.64 & 4.77 & 4.66 & 4.66 \\
\hline Free-Range Medium eggs (6-pack) & 4.71 & 4.60 & 4.60 & 4.66 & 4.55 & 4.55 & 4.67 & 4.57 & 4.57 & 4.74 & 4.62 & 4.62 \\
\hline Value Medium eggs (6-pack) & 4.73 & 4.63 & 4.63 & 4.69 & 4.58 & 4.58 & 4.67 & 4.57 & 4.57 & 4.74 & 4.63 & 4.63 \\
\hline Cola 2 I bottle & 5.50 & 5.40 & 5.40 & 5.57 & 5.48 & 5.48 & 5.25 & 5.14 & 5.14 & 5.44 & 5.34 & 5.34 \\
\hline Diet Cola 2 I bottle & 4.23 & 4.01 & 4.01 & 4.58 & 4.38 & 4.38 & 3.60 & 3.38 & 3.38 & 3.90 & 3.65 & 3.65 \\
\hline English Unsalted Butter & 5.48 & 5.38 & 5.38 & 5.28 & 5.18 & 5.18 & 5.45 & 5.35 & 5.35 & 5.54 & 5.44 & 5.44 \\
\hline English Salted Butter & 5.55 & 5.44 & 5.44 & 5.35 & 5.24 & 5.24 & 5.47 & 5.36 & 5.36 & 5.58 & 5.48 & 5.48 \\
\hline Cottage Pie 450g & 5.61 & 5.52 & 5.52 & 5.67 & 5.58 & 5.58 & 5.59 & 5.51 & 5.51 & 5.74 & 5.66 & 5.66 \\
\hline Light Choices Cottage Pie 450g & 5.42 & 5.33 & 5.33 & 5.49 & 5.40 & 5.40 & 5.36 & 5.28 & 5.28 & 5.50 & 5.42 & 5.42 \\
\hline Value Cottage Pie 450g & 5.64 & 5.55 & 5.55 & 5.73 & 5.65 & 5.65 & 5.51 & 5.43 & 5.43 & 5.66 & 5.57 & 5.57 \\
\hline
\end{tabular}

